\title{
Track and treat Parkinson's disease using wearable sensors and MRI
}

Citation for published version (APA):

Heijmans, M. H. M. (2021). Track and treat Parkinson's disease using wearable sensors and MRI.

[Doctoral Thesis, Maastricht University]. Maastricht University. https://doi.org/10.26481/dis.20211210mh

Document status and date:

Published: 01/01/2021

DOI:

10.26481/dis.20211210mh

Document Version:

Publisher's PDF, also known as Version of record

\section{Please check the document version of this publication:}

- A submitted manuscript is the version of the article upon submission and before peer-review. There can be important differences between the submitted version and the official published version of record.

People interested in the research are advised to contact the author for the final version of the publication, or visit the DOI to the publisher's website.

- The final author version and the galley proof are versions of the publication after peer review.

- The final published version features the final layout of the paper including the volume, issue and page numbers.

Link to publication

\footnotetext{
General rights rights.

- You may freely distribute the URL identifying the publication in the public portal. please follow below link for the End User Agreement:

www.umlib.nl/taverne-license

Take down policy

If you believe that this document breaches copyright please contact us at:

repository@maastrichtuniversity.nl

providing details and we will investigate your claim.
}

Copyright and moral rights for the publications made accessible in the public portal are retained by the authors and/or other copyright owners and it is a condition of accessing publications that users recognise and abide by the legal requirements associated with these

- Users may download and print one copy of any publication from the public portal for the purpose of private study or research.

- You may not further distribute the material or use it for any profit-making activity or commercial gain

If the publication is distributed under the terms of Article $25 \mathrm{fa}$ of the Dutch Copyright Act, indicated by the "Taverne" license above, 


\section{Track and treat Parkinson's disease using wearable sensors and MRI}

Margot Heijmans 
(C) Margot Heijmans, Maastricht 2021

Cover design: Margot Heijmans, Perla Douven, DrAfter123

Printed by: Ipskamp Printing

ISBN: 978-94-6423-529-6

All rights reserved. No part of this thesis may be reproduced, stored in a retrieval system of any nature, or transmitted in any form or by any means, electronic, mechanical, photocopying, recording, or otherwise, without permission of the author. 


\title{
Track and treat Parkinson's disease using wearable sensors and MRI
}

\author{
Proefschrift
}

Ter verkrijging van de graad van doctor aan de Universiteit Maastricht, op gezag van de Rector Magnificus, Prof. Dr. Rianne M. Letschert

volgens het besluit van het College van Decanen,

in het openbaar te verdedigen op vrijdag

10 december 2021 om 10.00 uur

door

Margot Helena Maria Heijmans 


\section{Promotor}

Prof. dr. Yasin Temel

\section{Copromotoren}

Dr. Pieter Kubben

Dr. Mark Kuijf

\section{Beoordelingscommissie}

Prof. dr. Marjolein de Vugt (voorzitter)

Prof. dr. Walter Backes

Prof. dr. Rob de Bie (Amsterdam UMC)

Dr. Rick Helmich (Radboudumc)

Dr. Kenneth Meijer

The research described in this thesis was performed at the School for Mental Health and Neuroscience, Department of Neurosurgery, Maastricht University, Maastricht, The Netherlands.

The research described in this thesis was sponsored by NFU Citrienfonds eHealth and Stichting De Weijerhorst. Printing of this thesis was kindly supported by de Parkinson Vereniging and Maastricht University.

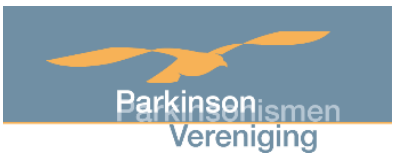




\section{Table of contents}

Chapter 1. General introduction

Part 1: Using wearable sensors to track and treat Parkinson's disease

Chapter 2. An update on adaptive deep brain stimulation in Parkinson's disease

Chapter 3. Monitoring Parkinson's disease symptoms during daily life: a feasibility study

Chapter 4. Evaluation of Parkinson's disease at home: Predicting tremor from wearable sensors

Chapter 5. The TRACK-PD study: protocol of a longitudinal ultra-high field imaging study in Parkinson's disease

Chapter 6. Comparison of olfactory tract diffusion measures between early Parkinson's disease patients and healthy controls using ultra-high field MRI

Chapter 7. Variability in subthalamic nucleus targeting for deep brain stimulation with 3 and 7 Tesla magnetic resonance imaging

Chapter 8. General discussion

Addendum. Impact paragraph

Addendum. Curriculum Vitae

Addendum. PhD portfolio

Addendum. Nederlandse samenvatting

Addendum. Dankwoord 

Chapter 1

General introduction 



\section{Parkinson's disease}

Parkinson's disease (PD) is the second most common neurodegenerative disorder (after Alzheimer's disease) with a prevalence around 1 per 100 of the population above $60^{1}$. The number of people with PD worldwide has doubled to over 6 million from 1990 to 2015. This number is thought to double again by 2040, making PD the fastest growing neurological disorder ${ }^{2}$. PD is a movement disorder characterized by slowness of movement (bradykinesia), muscle stiffness (rigidity) and resting tremor ${ }^{3,4}$. Besides these motor symptoms, most patients also have non-motor symptoms such as sleep disturbances, autonomic dysfunction, hyposmia, cognitive decline and psychiatric symptoms. These nonmotor symptoms often precede the motor symptoms by many years ${ }^{5,6}$. The aetiology of PD is still largely unknown, however it is thought that most of the early (non-motor) symptoms depend on progressive dopaminergic neurodegeneration originating in the brainstem. The motor symptoms are attributed to the later occurring dopaminergic neurodegeneration in the substantia nigra pars compacta ${ }^{7}$. The degeneration of dopaminergic neurons eventually leads to a dopamine deficiency in the PD brain.

\section{Parkinson as heterogeneous disorder}

'Both my parents have PD. Although they are diagnosed with the same disease, their PD symptoms are completely different. My dad's hands are already shaking for over 10 years. He responded well at the medication at first, but while the disease progressed, his hands are shaking more often despite medication intake. My mom showed less facial expression at some point and movements in general, like walking, writing and eating, became much slower. I am participating to this study since I have seen that PD is such a complicated disease with a varying symptomatology for each single person diagnosed with it.' - Participant TRACKPD study

PD has a considerable variation of dominant motor and non-motor symptoms. One may for example mainly suffer from a resting tremor, while another may never develop tremor and mostly suffer from postural instability. In addition, one may experience many autonomic dysfunctions during his or her disease course, while another may specifically experience cognitive decline. The observation of PD being such a heterogeneous disorder has led to a subdivision of PD patients into various phenotypes ${ }^{8}$. Not only the dominant 
symptoms vary per PD patient, but also the progression rate. Since every PD patient is unique, it is impossible to predict how an individual's PD symptoms will progress over time. The underlying aetiology of the heterogeneity in PD is not well understood ${ }^{9}$. Interestingly, observations pointed towards different deficit levels of the striato-thalamo-cortical and cerebello-thalamo-cortical circuity between PD subgroups ${ }^{10}$.

\section{Track Parkinson's disease}

'At first I went to my general practitioner with some shoulder complaints. He sent me to the physiotherapist, who called it a frozen shoulder. Physiotherapy didn't really work, so I quit this after some months. Half a year later, my partner told me she couldn't hear me that well lately. Also my handwriting became smaller and smaller over time. Again I went to the general practitioner and told him about my complaints. This time he sent me to the neurologist. The neurologist looked thoroughly at me while I was talking and he asked me to walk up and down the hallway a few times. Eventually he asked me about my sense of smell. At that moment I realised that I had gradually lost my sense of smell already some years ago. He thought I had PD. After a DaT-scan he confirmed the diagnosis of PD. This was about one and a half year after my first visit to the general practitioner.' Participant TRACK-PD study

The error rate for a clinical diagnosis of PD can be as high as $24 \%$, even in specialized centers 11. There are several reasons for this. Diagnosing PD can be challenging since mild symptoms are often not immediately recognized. In addition, there are several atypical parkinsonian disorders, such as progressive supranuclear palsy, multiple system atrophy and corticobasal degeneration, which show parkinsonian symptoms and are challenging to clinically separate from PD ${ }^{12}$. Parkinsonian symptoms can also be drug-induced and also here, differentiating drug-induced parkinsonism from PD can be challenging on a clinical ground ${ }^{13}$. Diagnosing PD is currently done by assessing clinical symptoms and their course over time ${ }^{4}$. New clinical criteria for diagnosing PD have been recently published on behalf of the International PD and Movement Disorders Society ${ }^{4}$ and provide an overview of supportive criteria, absolute exclusion criteria, and red flags. Magnetic Resonance Imaging (MRI) scans are often made to exclude other potential causes of parkinsonian symptoms, 
like for example extensive vascular damage in the basal ganglia. A Dopamine Transporter Scan (DaT-scan) can be performed when doubts remain regarding the diagnosis of PD, especially in differentiating essential tremor overlapping syndromes or drug-induced parkinsonism. In this test, a radioactive tracer is injected into the blood which will make its way into the brain. After several hours the actual scan is made which visualizes the radioactive tracer. Presence of the tracer has shown to be reduced in the early stages of PD compared with healthy controls, and a DaT-scan therefore is leading in case of doubt about the diagnosis of PD. The sensitivity of a DaT-scan to detect PD is still suboptimal ${ }^{14,15}$. Other disadvantages are that it is more invasive compared to for example MRI and it is time and money consuming. The subjectivity in assessing clinical symptoms, combined with the heterogeneity of PD and the lacking sensitivity of the DaT-scan unfortunately often leads to a long, unsure trajectory for the patient.

Although MRI scans are now only made to exclude other potential causes of parkinsonian symptoms, several imaging features are considered valuable for assisting in diagnosing PD. There are already several promising imaging features known, in especially structural MRI, which differ between PD patients compared to healthy controls ${ }^{16,17}$. There is signal loss in the largest subgroup of dopamine-containing neurons in the substantia nigra (nigrosome1 area) in PD patients compared to healthy controls on iron sensitive MR images ${ }^{18}$. On neuromelanin sensitive images, there is also reduced signal intensity of the substantia nigra ${ }^{19}$. On diffusion weighted images, it has been shown that there is more free-water in the substantia nigra of PD patients compared to healthy controls ${ }^{20}$. A recent meta-analysis highlights the corticospinal tract and the olfactory regions as regions which might show differences between PD patients and healthy controls ${ }^{21}$.

The various differences in imaging features on themselves seem to lack sensitivity to serve as a discriminating tool to detect PD. Therefore, the focus is more and more on studies combining various MR scans (multimodal MRI) and their corresponding imaging features ${ }^{22}$. Another development which could help to increase the sensitivity of imaging features is the emergence of ultra-high-field MRI (7 Tesla and above). With ultra-high-field MRI, submillimeter anatomical information with increased contrast can be obtained ${ }^{23}$. The increased spatial resolution that ultra-high-field MRI offers might enable a higher degree of diagnostic detail when comparing imaging features between PD patients and healthy controls. 


\section{Treat Parkinson's disease}

'Since I am receiving deep brain stimulation I am a totally different person. I have way more energy and my movements are more smoother and quicker. In addition,

my tremor has substantially reduced. I now have two different programs from which I can choose. One programme I use when I am at home with my family. With this programme I get less stimulation, which is optimal for my speech, but suboptimal for the suppression of tremor. With the other programme my tremor is more or less suppressed, but my speech becomes slurred. I am glad I can choose between those two programs.' - Participant Monitoring at home study

Until now, there is no cure for PD. The dopamine deficiency can be supplemented with levodopa, which can bring an important improvement of PD symptoms. After 4-6 years of disease progression, patients might experience variation in symptom expression over the day 24,25 , which can be reduced with medication adjustment. Unfortunately, with progression of the disease, other adverse effects may emerge, such as medication related dyskinesias or psychiatric complications. Patients also may experience response fluctuations; an alternation between episodes in which the effect of the medication is noticeable (ON-phase) and episodes in which the symptoms reoccur due to less or no beneficial effect of medication (OFF-phase).

An established treatment for advanced PD is Deep Brain Stimulation (DBS). Since its introduction in the 1990s, DBS of the subthalamic nucleus (STN) has been shown to be an effective surgical treatment for PD ${ }^{26,27}$. DBS for PD is especially efficacious in treating otherwise refractory tremor, motor fluctuations and dyskinesias ${ }^{28,29}$. Despite these positive outcomes, STN DBS is also accompanied by possible side-effects, like behavioural changes and cognitive, speech, balance and gait problems ${ }^{30}$. The precision of electrode targeting is considered as one of the most important factors determining DBS outcome. The most common practice for targeting the STN for DBS is by using T2-weighted MRI images. These images, which are sensitive to iron content, will show the STN, which is an iron rich structure, as hypointense. Traditionally, STN targeting is done by using lower field MRI images (1.5 and 3 Tesla MRI). Images created by these field strengths result in images that lack sharp and clear borders of small deep brain structures ${ }^{31}$. Instead, ultra-high-field MRI can obtain submillimeter anatomical information with increased contrast ${ }^{23}$. Recent 
research shows that ultra-high field imaging offers more easy targeting and might eventually play a role in optimizing the precision of electrode targeting ${ }^{32}$. Whether the benefits of Ultra-high-field MRI results in better targeting for DBS stays however unclear 32-34.

To adjust or optimize treatment by medication as well as DBS, symptoms and the disease course are charted by a clinician during visits to the outpatient clinic. This has some limitations; 1) The assessment only takes place once per couple of months. It therefore only permits a snapshot of the clinical situation and might include recall bias. 2) The assessment has a subjective character, since the clinician describes or scores the complaints of the patient and the execution of movement tasks. 3) PD patients might put themselves in a better light during in-clinical assessments. Because of these reasons, more frequent and objective ratings of symptoms and the disease course are essential to obtain a better treatment for PD patients. Eventually, frequent and objective ratings might also be used as input for adaptive, or closed-loop DBS.

Wearable sensors are more and more used to detect PD symptoms during daily life. Such sensors mostly contain an accelerometer and gyroscope, measuring respectively acceleration and rotational acceleration of the body part the sensor is attached to in the $x$, $y$, and $z$ direction. Wearable sensors have shown potential in monitoring PD symptoms like tremor ${ }^{35-37}$, freezing of gait ${ }^{38}$, bradykinesia ${ }^{39}$ and dyskinesia ${ }^{39,40}$. In addition to wearable sensors, standard diary methods can be used in monitoring systems to also include subjective scores given by the patient self. A more recently used digital diary method is the Experience Sampling Method (ESM). ESM is a validated method consisting of multiple repeated measurements at semi-random moments in daily life. It does not allow any recall bias, since the user has to complete the questionnaire at the moment it pops up ${ }^{41}$. ESM has shown to be feasible in PD patients ${ }^{42,43}$ and the data may serve as a parallel ground truth to wearable sensor data in monitoring systems.

\section{Outline of this thesis}

This thesis is divided into two parts. The first part focuses on the role wearable sensors can play in especially treating PD and the second part focuses on the role of MRI in tracking and treating PD.

\section{Part 1: Using wearable sensors to track and treat Parkinson's disease}

Chapter 2 provides a narrative review on adaptive deep brain stimulation for PD. In this review, the concept of adaptive deep brain stimulation is explained, and the potential role wearable sensors can play in adaptive deep brain stimulation is discussed.

In Chapter $\mathbf{3}$ we evaluated whether wearable sensors can be used in combination with the experience sampling method for two consecutive weeks to track PD symptoms at home. 
Furthermore, we propose how to analyse and work further with the new combination of these different data modalities.

In Chapter 4 we evaluated whether we could predict a subjective tremor score given by a patient, based on parameters extracted from the wearable sensor data.

\section{Part 2: Using MRI to track and treat Parkinson's disease}

Chapter 5 provides the detailed protocol of our longitudinal ultra-high field imaging (7T) study in PD; the TRACK-PD study.

In Chapter 6 we evaluated whether we could replicate previous findings which showed that diffusion measures of the olfactory tract differ between PD patients and healthy controls. We aimed to replicate previous findings using 7T MRI TRACK-PD data of early PD patients.

In Chapter 7 we evaluated whether using ultra-high field 7T MRI data would lead to less variability compared to $3 \mathrm{~T} \mathrm{MRI}$ data when targeting the subthalamic nucleus for deep brain stimulation in PD.

Finally, in Chapter 8 a summary of the main findings will be presented, the most relevant findings will be discussed and integrated, and future directions will be highlighted.

'On my way back home from the testing day, I just realized how special it is that young people like you are so engaged in doing research to ' $m y^{\prime}$ disease. I think that really gives hope!' - Participant TRACK-PD study 


\section{References}

1. Nussbaum, R. L. \& Ellis, C. E. Alzheimer's disease and Parkinson's disease. N. Engl. J. Med. 348, 13561364 (2003).

2. Dorsey, E. R., Sherer, T., Okun, M. S. \& Bloem, B. R. The Emerging Evidence of the Parkinson Pandemic. J. Park. Dis. 8, S3-S8 (2018).

3. Jankovic, J. Parkinson's disease: clinical features and diagnosis. J. Neurol. Neurosurg. Psychiatry 79, 368-376 (2008).

4. Postuma, R. B. et al. MDS clinical diagnostic criteria for Parkinson's disease. Mov. Disord. Off. J. Mov. Disord. Soc. 30, 1591-1601 (2015).

5. Abbott, R. D. et al. Frequency of bowel movements and the future risk of Parkinson's disease. Neurology 57, 456-462 (2001).

6. Khoo, T. K. et al. The spectrum of nonmotor symptoms in early Parkinson disease. Neurology $80,276-$ 281 (2013).

7. Kish, S. J., Shannak, K. \& Hornykiewicz, O. Uneven pattern of dopamine loss in the striatum of patients with idiopathic Parkinson's disease. Pathophysiologic and clinical implications. N. Engl. J. Med. 318, 876-880 (1988).

8. Fereshtehnejad, S.-M., Zeighami, Y., Dagher, A. \& Postuma, R. B. Clinical criteria for subtyping Parkinson's disease: biomarkers and longitudinal progression. Brain J. Neurol. 140, 1959-1976 (2017).

9. Thenganatt, M. A. \& Jankovic, J. Parkinson disease subtypes. JAMA Neurol. 71, 499-504 (2014).

10. Boonstra, J. T., Michielse, S., Temel, Y., Hoogland, G. \& Jahanshahi, A. Neuroimaging Detectable Differences between Parkinson's Disease Motor Subtypes: A Systematic Review. Mov. Disord. Clin. Pract. 8, 175-192 (2021).

11. Hughes, A. J., Daniel, S. E., Ben-Shlomo, Y. \& Lees, A. J. The accuracy of diagnosis of parkinsonian syndromes in a specialist movement disorder service. Brain J. Neurol. 125, 861-870 (2002).

12. Williams, D. R. \& Litvan, I. Parkinsonian Syndromes. Contin. Lifelong Learn. Neurol. 19, 1189-1212 (2013).

13. Brigo, F., Erro, R., Marangi, A., Bhatia, K. \& Tinazzi, M. Differentiating drug-induced parkinsonism from Parkinson's disease: an update on non-motor symptoms and investigations. Parkinsonism Relat. Disord. 20, 808-814 (2014).

14. Oravivattanakul, S. et al. Dopamine Transporter (DaT) Scan Utilization in a Movement Disorder Center. Mov. Disord. Clin. Pract. 3, 31-35 (2016).

15. Poewe, W. \& Scherfler, C. Role of dopamine transporter imaging in investigation of parkinsonian syndromes in routine clinical practice. Mov. Disord. Off. J. Mov. Disord. Soc. 18 Suppl 7, S16-21 (2003).

16. Lehericy, S. et al. The role of high-field magnetic resonance imaging in parkinsonian disorders: Pushing the boundaries forward. Mov. Disord. Off. J. Mov. Disord. Soc. 32, 510-525 (2017).

17. Prange, S., Metereau, E. \& Thobois, S. Structural Imaging in Parkinson's Disease: New Developments. Curr. Neurol. Neurosci. Rep. 19, 50 (2019).

18. Schwarz, S. T. et al. The 'Swallow Tail' Appearance of the Healthy Nigrosome - A New Accurate Test of Parkinson's Disease: A Case-Control and Retrospective Cross-Sectional MRI Study at 3T. PLoS ONE 9, (2014).

19. Isaias, I. U. et al. Neuromelanin Imaging and Dopaminergic Loss in Parkinson's Disease. Front. Aging Neurosci. 8, 196 (2016).

20. Ofori, E. et al. Increased free-water in the substantia nigra of Parkinson's disease: a single-site and multi-site study. Neurobiol. Aging 36, 1097-1104 (2015).

21. Atkinson-Clement, C., Pinto, S., Eusebio, A. \& Coulon, O. Diffusion tensor imaging in Parkinson's disease: Review and meta-analysis. Neurolmage Clin. 16, 98-110 (2017).

22. Esterhammer, R. et al. Potential of Diffusion Tensor Imaging and Relaxometry for the Detection of Specific Pathological Alterations in Parkinson's Disease (PD). PloS One 10, e0145493 (2015).

23. Inglese, M., Fleysher, L., Oesingmann, N. \& Petracca, M. Clinical applications of ultra-high field magnetic resonance imaging in multiple sclerosis. Expert Rev. Neurother. 18, 221-230 (2018).

24. Aquino, C. C. \& Fox, S. H. Clinical spectrum of levodopa-induced complications. Mov. Disord. Off. J. Mov. Disord. Soc. 30, 80-89 (2015).

25. Calabresi, P., Di Filippo, M., Ghiglieri, V., Tambasco, N. \& Picconi, B. Levodopa-induced dyskinesias in patients with Parkinson's disease: filling the bench-to-bedside gap. Lancet Neurol. 9, 1106-1117 (2010). 
26. Krauss, J. K. et al. Technology of deep brain stimulation: current status and future directions. Nat. Rev. Neurol. (2020) doi:10.1038/s41582-020-00426-z.

27. Limousin, P. et al. Bilateral subthalamic nucleus stimulation for severe Parkinson's disease. Mov. Disord. Off. J. Mov. Disord. Soc. 10, 672-674 (1995).

28. Deuschl, G. et al. A randomized trial of deep-brain stimulation for Parkinson's disease. N. Engl. J. Med. 355, 896-908 (2006).

29. Limousin, P. \& Foltynie, T. Long-term outcomes of deep brain stimulation in Parkinson disease. Nat. Rev. Neurol. 15, 234-242 (2019).

30. Temel, Y. et al. Behavioural changes after bilateral subthalamic stimulation in advanced Parkinson disease: a systematic review. Parkinsonism Relat. Disord. 12, 265-272 (2006).

31. Forstmann, B. U., Isaacs, B. R. \& Temel, Y. Ultra High Field MRI-Guided Deep Brain Stimulation. Trends Biotechnol. 35, 904-907 (2017).

32. Bot, M. et al. Defining the Dorsal STN Border Using 7.0-T MRI: A Comparison to Microelectrode Recordings and Lower Field Strength MRI. Stereotact. Funct. Neurosurg. 97, 153-159 (2019).

33. Bot, M. et al. Deep brain stimulation for Parkinson's disease: defining the optimal location within the subthalamic nucleus. J. Neurol. Neurosurg. Psychiatry 89, 493-498 (2018).

34. Springer, E. et al. Comparison of Routine Brain Imaging at 3 T and 7 T. Invest. Radiol. 51, 469-482 (2016).

35. Basu, I. et al. Pathological tremor prediction using surface electromyogram and acceleration: potential use in 'ON-OFF' demand driven deep brain stimulator design. J. Neural Eng. 10, 036019 (2013).

36. Delrobaei, M. et al. Towards remote monitoring of Parkinson's disease tremor using wearable motion capture systems. J. Neurol. Sci. 384, 38-45 (2018).

37. Khobragade, N., Graupe, D. \& Tuninetti, D. Towards fully automated closed-loop Deep Brain Stimulation in Parkinson's disease patients: A LAMSTAR-based tremor predictor. Annu. Int. Conf. IEEE Eng. Med. Biol. Soc. IEEE Eng. Med. Biol. Soc. Annu. Int. Conf. 2015, 2616-2619 (2015).

38. Rodríguez-Martín, D. et al. Home detection of freezing of gait using support vector machines through a single waist-worn triaxial accelerometer. PloS One 12, e0171764 (2017).

39. Griffiths, R. I. et al. Automated assessment of bradykinesia and dyskinesia in Parkinson's disease. J. Park. Dis. 2, 47-55 (2012).

40. Delrobaei, M., Baktash, N., Gilmore, G., Mclsaac, K. \& Jog, M. Using Wearable Technology to Generate Objective Parkinson's Disease Dyskinesia Severity Score: Possibilities for Home Monitoring. IEEE Trans. Neural Syst. Rehabil. Eng. Publ. IEEE Eng. Med. Biol. Soc. 25, 1853-1863 (2017).

41. Palmier-Claus, J. E. et al. Experience sampling research in individuals with mental illness: reflections and guidance. Acta Psychiatr. Scand. 123, 12-20 (2011).

42. Broen, M. P. G. et al. Unraveling the Relationship between Motor Symptoms, Affective States and Contextual Factors in Parkinson's Disease: A Feasibility Study of the Experience Sampling Method. PloS One 11, e0151195 (2016).

43. Mulders, A. E. P. et al. Usability of the Experience Sampling Method in Parkinson's Disease on a Group and Individual Level. Mov. Disord. Off. J. Mov. Disord. Soc. 35, 1145-1152 (2020). 
Part 1: Using wearable sensors to track and treat Parkinson's disease 



\title{
Chapter 2
}

\section{An update on adaptive deep brain stimulation in Parkinson's disease}

\author{
J.G.V. Habets*, M. Heijmans*, M.L. Kuijf, M.L.F. Janssen, Y. Temel, P.L. Kubben \\ * shared first authors
}

Published. Movement Disorders 2018; 33(12):1834-1843 


\begin{abstract}
Advancing conventional open-loop DBS as a therapy for PD is crucial for overcoming important issues such as the delicate balance between beneficial and adverse effects and limited battery longevity that are currently associated with treatment. Closed-loop or adaptive DBS aims to overcome these limitations by real-time adjustment of stimulation parameters based on continuous feedback input signals that are representative of the patient's clinical state. The focus of this update is to discuss the most recent developments regarding potential input signals and possible stimulation parameter modulation for adaptive DBS in PD. Potential input signals for adaptive DBS include basal ganglia local field potentials, cortical recordings (electrocorticography), wearable sensors, and eHealth and mHealth devices. Furthermore, adaptive DBS can be applied with different approaches of stimulation parameter modulation, the feasibility of which can be adapted depending on specific PD phenotypes. Implementation of technological developments like machine learning show potential in the design of such approaches; however, energy consumption deserves further attention. Furthermore, we discuss future considerations regarding the clinical implementation of adaptive DBS in PD.
\end{abstract}

\title{
Keywords
}

Adaptive; closed-loop; deep brain stimulation; Parkinson's disease; stimulation paradigms 
Conventional deep brain stimulation (cDBS) of the subthalamic nucleus (STN) or the globus pallidus internus (GPi) is an established treatment for advanced stage Parkinson's disease (PD). Although cDBS improves the motor symptoms of PD in both the short and long term, it is not without limitations ${ }^{1,2}$. Stimulation induced side effects such as dysarthria ${ }^{3}$, imbalance, and dyskinesia can occur and often require regular adjustments in stimulation, especially in the first phase after surgery ${ }^{4}$. Moreover, cDBS has limited battery life. These limitations have led to development and expanding scientific interest in closed-loop, responsive, or adaptive DBS (aDBS; Fig. 1). For consistency reasons, only the term aDBS will be used.

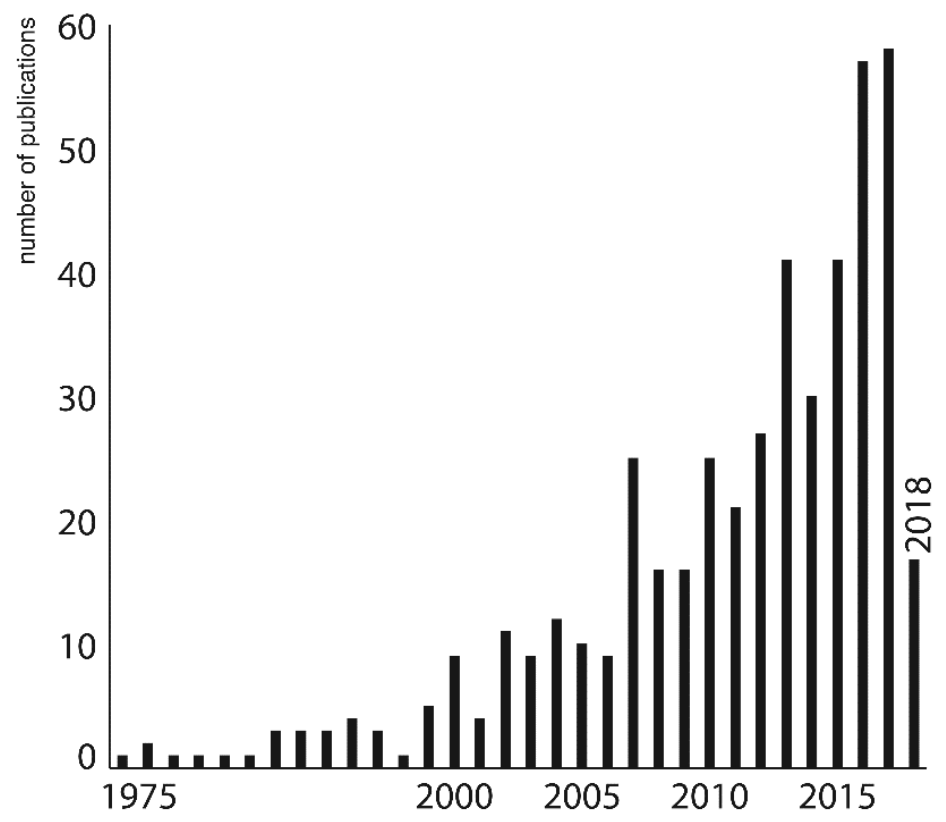

Figure 1. Yearly number of publications on aDBS in PD, searched on PubMed on 5-3-2018, using search command: [(parkinson*) AND (adaptive OR (closed loop) OR (closed-loop) OR responsive) AND (dbs OR stimulation)].

In cDBS, stimulation parameters are traditionally programmed and evaluated by a clinician during outpatient visits. If necessary, stimulation parameters are adjusted, and patients can perform minor changes within preset ranges themselves later. The goal of aDBS is to optimize this process further and automatically adapt stimulation parameters to the fluctuating clinical state of the patient, where, in theory, stimulation is given only when necessary. As such, aDBS may generate fewer side effects attributed to the possible decrease in energy given. In addition, although more power may be needed for data processing, the required battery consumption for stimulation potentially decreases and could result in increased battery longevity. Clinical proof-of-concept studies have already shown beneficial results using electrophysiological and/or wearable sensor recordings as 
feedback signals for aDBS in PD ${ }^{5,6}$. The next step is to confirm whether such an approach continues its efficacy in the long term and discuss new issues on the design of aDBS.

Development of a valid aDBS system in PD faces major challenges such as creating suitable input and processing input signals into beneficial output. In the following sections, we present an update, future needs and possibilities for input signals, and stimulation paradigms for aDBS in PD. Much of the technological and clinical knowledge and experience discussed here also relates to the use of aDBS in other fluctuating neurological and psychiatric diseases, such as essential tremor (ET), dystonia, epilepsy, Tourette's syndrome, and obsessive-compulsive disorder.

\section{Potential input signals for aDBS}

To develop a valid aDBS system, robust input signals representing the main PD symptoms are needed (Fig. 2). Symptoms vary from patient to patient, and therefore the suitability of input signals differs for individual patients (Fig. 3). Furthermore, the necessity of supplementary (non-)invasive implants or devices, as well as additional processing and computational demands, should be taken into consideration when comparing input signals. A comprehensive and concise overview of rationales and basic principles regarding potential input signals has recently been reviewed elsewhere ${ }^{7}$. We will elaborate further on previous work by discussing up-to-date progress and the remaining challenges regarding potential input signals for aDBS in PD.

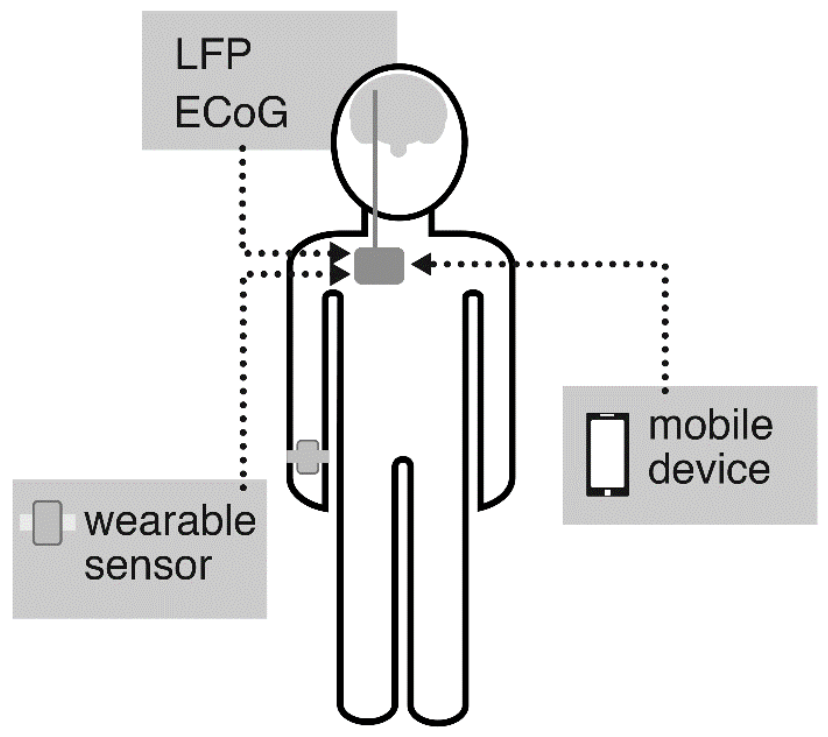

Figure 2. Schematic overview of the most used possible input signal origins for aDBS in PD. Sensors can also be worn on different locations, for example, the chest, legs, or fingers. 


\section{aDBS based on electrophysiological recordings}

\section{Basal ganglia recordings}

New-generation DBS pulse generators can record local field potentials (LFPs), which have been correlated with clinical symptoms in several studies. For example, decreased beta band (8-35 Hz) activity in the STN by dopaminergic medication and/or DBS has been correlated with improved akinesia, bradykinesia, and rigidity ${ }^{8,9}$, but not with tremor ${ }^{10-12}$. However, others found a correlation between STN-LFP recordings and tremor 13,14. Furthermore, freezing-of-gait periods 15 and differentiation between speech and movement activities ${ }^{16}$ can be detected using STN-LFPs. Such differentiation of clinical indications underlines the potential of STN-LFP recordings as promising input signals, with the added benefit of not requiring additional implants or equipment compared to cDBS ${ }^{17}$.

A number of proof-of-concept studies using beta-LFPs to modify aDBS have shown motor improvement ${ }^{5,18}$ less speech impairment ${ }^{19}$, and less levodopa-induced dyskinesia compared to $\mathrm{CDBS}^{20}$, which suggests that this is a more efficient and effective method of stimulation. Moreover, a recent study demonstrated the feasibility and beneficial effects on motor symptoms of aDBS over the course of 8 hours in akinetic-rigid PD patients ${ }^{21}$. Previous studies had already shown that aDBS was applicable and effective in a freely moving ${ }^{22}$ and a chronically implanted PD patient ${ }^{23}$.

Nevertheless, beta-LFPs in the STN are not (easily) detectable in all patients, 8 although this long-standing assumption has been contradicted recently ${ }^{24}$. Second, changes in beta-LFPs do not clearly capture all main symptoms of PD. For example, the relationship with tremor is debated. Although other STN-LFP signals like theta-band $(3-8 \mathrm{~Hz})$ activity show promise in relation to tremor ${ }^{13}$, additional input signals to monitor tremor might be needed ${ }^{24,25}$. Third, the clinical relevance or symptomatic contribution of high-versus low-beta bands is a topic of discussion ${ }^{26,27}$. Last, alpha/betaband activity is influenced by daily life events such as rest tremor ${ }^{24}$, voluntary movements ${ }^{28,29}$, movement artifacts during gait ${ }^{30}$, different vigilance states and sleep ${ }^{31}$, and aDBS itself ${ }^{32}$, which makes the isolation of disease-related signals difficult.

Despite these challenges, basal ganglia LFPs have been shown to function as a suitable input signal for aDBS. The main challenge to enable clinical use of LFPs is the development of standardized techniques that allow for automatic and validated interpretation of input signals. Therefore, further development of the hardware and software of aDBS systems is needed to acquire various frequency bands or additional input signals. Eventually, these sophisticated aDBS systems should better suit the difference in clinical needs between akinetic-rigid and tremor-dominant PD patients. 


\begin{tabular}{|c|c|c|c|c|c|}
\hline $\begin{array}{l}\text { Input signal vs. } \\
\text { Parkinsonian } \\
\text { symptoms }\end{array}$ & $\begin{array}{l}\text { Correlation } \\
\text { w/ tremor }\end{array}$ & $\begin{array}{l}\text { Correlation } \\
\text { w/ bradykinesia/ } \\
\text { rigidity }\end{array}$ & $\begin{array}{l}\text { Correlation } \\
\text { w/ (freezing of) gait }\end{array}$ & $\begin{array}{l}\text { Correlation } \\
\text { w/ dyskinesia }\end{array}$ & $\begin{array}{l}\text { Correlation } \\
\text { w/ non-motor } \\
\text { symptoms }\end{array}$ \\
\hline $\begin{array}{l}\text { Subcortical } \\
\text { recordings } \\
(\mathrm{STN} L F P)\end{array}$ & $\begin{array}{l}\text { repeated reports } \\
\text { small evidence } \\
\text { on debate }\end{array}$ & $\begin{array}{l}\text { repeated reports } \\
\text { reproduced evidence } \\
\text { starting consensus }\end{array}$ & $\begin{array}{l}\text { first reports } \\
\text { small evidence } \\
\text { no consensus }\end{array}$ & $\begin{array}{l}\text { first reports } \\
\text { small evidence } \\
\text { no consensus }\end{array}$ & $\begin{array}{l}\text { first reports } \\
\text { no evidence } \\
\text { no consensus }\end{array}$ \\
\hline $\begin{array}{l}\text { Cortical recordings } \\
\text { (ECoG) }\end{array}$ & $\begin{array}{l}\text { first reports } \\
\text { no evidence } \\
\text { no consensus }\end{array}$ & $\begin{array}{l}\text { repeated reports } \\
\text { reproduced evidence } \\
\text { starting consensus }\end{array}$ & not possible yet* & $\begin{array}{l}\text { first reports } \\
\text { small evidence }\end{array}$ & not possible yet * \\
\hline $\begin{array}{l}\text { Wearable sensors } \\
\text { (accelerometer, } \\
\text { gyroscope) }\end{array}$ & $\begin{array}{l}\text { repeated reports } \\
\text { reproduced evidence } \\
\text { starting consensus }\end{array}$ & $\begin{array}{l}\text { first reports } \\
\text { small evidence } \\
\text { no consensus }\end{array}$ & $\begin{array}{l}\text { first reports } \\
\text { small evidence } \\
\text { no consensus }\end{array}$ & $\begin{array}{l}\text { first reports } \\
\text { small evidence } \\
\text { no consensus }\end{array}$ & not possible yet \\
\hline Mobile application & $\begin{array}{l}\text { first reports } \\
\text { no evidence } \\
\text { no consensus }\end{array}$ & $\begin{array}{l}\text { repeated reports } \\
\text { no evidence } \\
\text { no consensus }\end{array}$ & $\begin{array}{l}\text { first reports } \\
\text { no evidence } \\
\text { no consensus }\end{array}$ & $\begin{array}{l}\text { first reports } \\
\text { no evidence } \\
\text { no consensus }\end{array}$ & $\begin{array}{l}\text { repeated reports } \\
\text { small evidence } \\
\text { no consensus }\end{array}$ \\
\hline & $D \bigcirc \bigcirc$ & & $\mathbf{D} \bigcirc \bigcirc$ & $D \bigcirc \bigcirc$ & \\
\hline
\end{tabular}

* In Parkinsonian patients, repeatedly reported with small evidence in other diseases.

Figure 3. Overview of published evidence of the feasibility of different input signals regarding different parkinsonian symptoms for aDBS in PD. All input signals are scored on three categories per symptom. For each category $0,0.5$, or 1 bullet is given and the sum of them is visualized. The first line indicates the amount of publications: not possible yet (0), first reports (0.5), and repeated reports (1). The second line indicates the quality of reported evidence: no evidence (0), small evidence (0.5), and reproduced evidence (1). The third line indicates the amount of consensus on the use of an input signal for a symptom: no consensus (0), on debate (0.5), and starting consensus (1). 


\section{Cortical Recordings}

A hallmark of PD is pathological hyperactivity of the corticobasal pathways, which is attributed to dopamine denervation of the striatum and substantia nigra ${ }^{33}$. This hyperactivity results in clinically identifiable cortical oscillations, which can be measured invasively by electrocorticography (ECOG) using a subdural grid. aDBS can utilize these cortical oscillations as an input signal. For instance, one study showed that GPi-aDBS in nonhuman primates based on motor cortex (M1) beta activity resulted in beneficial effects on akinesia ${ }^{34}$. Spatial-specific attenuation of cortical beta hypersynchrony was also demonstrated in humans subsequent to STN-DBS .35 Recent studies use phase-amplitude coupling (PAC), whereby the amplitude of specific bandwidth oscillations is coupled to specific oscillation phases. In akinetic-rigid PD patients, excessiveM1-beta-gamma-PAC decreased during STN-DBS, parallel to a decrease of clinically assessed bradykinesia ${ }^{35,36}$. In contrast, in tremor-dominant PD patients, excessive M1-beta-PAC decreased during rest tremor ${ }^{37}$. Moreover, ECoG recordings showed potential to monitor dyskinesia ${ }^{38}$, gait characteristics, such as walking duration and speed ${ }^{39}$, and to perform speech recognition 40. Interpretation of cortical PAC values therefore requires differentiation between phenotypic manifestations.

This work led to use of a fully implanted ECoG based aDBS device in PD patients who experienced moderate dyskinesia despite optimized STN-DBS therapy ${ }^{41}$. The researchers adjusted stimulation voltage based on gamma-band $(60-90 \mathrm{~Hz})$ activity, which is related to dyskinesia. The clinical effect on bradykinesia and dyskinesia was maintained, while energy savings were $\sim 40 \%$.

A remaining concern is the limited correlation with PD symptoms and PAC attenuation attributed to movement preparation and execution ${ }^{36}$. Equal to beta-LFP in the STN, cortical beta-PAC is altered by DBS, which has implications for the analytical process ${ }^{42}$. Moreover, the time-frequency method used most often in PAC analysis might cause artifacts attributed to ignorance of the existence of both harmonic and non-sinusoidal neural dynamics in PD ${ }^{43}$. Another concern is that implantation of subdural grids may be associated with increased risk of complications, such as hemorrhage and infection. As recently demonstrated, use of cortical PAC is promising because of its potential ability to decode movement and behavior. Therefore, further steps are warranted to integrate the analyzed information from PAC and develop analytic algorithms for different PD symptoms to perform aDBS based on cortical recordings in the whole PD spectrum.

\section{Surface electromyography}

For several decades, surface electromyography (sEMG) signals have been used in tremor detection and more recently in tremor prediction ${ }^{44-47}$. Therefore, sEMG is considered to be a potential input signal for aDBS for ET and tremor-dominant PD. SEMG-based aDBS was 
feasible, effective, and efficient in ET patients ${ }^{48-50}$. Given that evidence of sEMG-based bradykinesia and rigidity detection methods is limited ${ }^{51,52}$, sEMG should be combined with other input signals for akinetic-rigid PD patients. Another major concern of sEMG-based aDBS is potential loss of data quality attributed to the required self-management of sEMG sensors by patients. Furthermore, signals must be processed and transmitted wirelessly to the pulse generator, which, in turn, may limit its battery life. To overcome these disadvantages, wireless sEMG sensors should be developed to withstand high contact impedances by using, for example, interchangeable patches to attach them to the skin or subcutaneous implantable EMG electrodes. However, the limited potential of SEMG as an input signal and the current progress in wearable sensor development seem to make sEMG impractical for aDBS in PD.

\section{aDBS based on neurochemical recordings}

As stated in previous work, development of aDBS based on neurochemical recordings is in an early phase ${ }^{7}$. Artifact-free neurochemical recordings were possible during DBS in rodents ${ }^{53}$, and dopamine fluctuations depending on DBS were found ${ }^{54}$. Therefore, neurochemical recordings were regarded to be a potential input signal for aDBS; however, the relationship between neurochemical recordings, PD symptoms, and DBS in humans has not been explored. Because no progress has been reported recently, limitations for clinical use of neurochemical feedback in aDBS remain substantial.

\section{aDBS based on wearable sensors}

Monitoring PD symptoms through wearable sensors, or "wearables," containing accelerometers and/or gyroscopes has gained considerable interest, and important progress has been made in the last decade ${ }^{55}$. Wearables are successful in predicting and detecting tremor ${ }^{44,46,56}$ and show promise in assessing freezing of gait ${ }^{57}$, bradykinesia, and dyskinesia ${ }^{58-60}$.

Numerous studies based on tremor detection have supported the feasibility, effectiveness, and efficiency of wearables-based aDBS ${ }^{6,49,61}$. However, no other PD symptoms are yet detectable or implemented with wearable aDBS systems, and therefore the applicability for akinetic-rigid PD patients is unclear ${ }^{61}$.

Application of wearables for aDBS will rely heavily on machine-learning approaches for distinguishing symptoms from voluntary movements ${ }^{62}$. Another concern is that patients will need to wear the sensors almost chronically. However, given that sensors are getting smaller and more aesthetically attractive, this might not be a problem for all. In addition, continuous assessment of PD symptoms at home using wearables does not affect healthrelated quality of life ${ }^{63}$. Last, signal processing and wireless data transmission may limit battery life of wearables and pulse generators. 
To implement aDBS controlled by wearables, algorithms to monitor other cardinal motor symptoms than tremor need further development and clinical validation to expand the potential for akinetic-rigid PD patients. For tremor-dominant patients, clinical trials with longer follow-up periods should be done to prove superiority compared to cDBS.

\section{aDBS based on PD monitoring systems including eHealth and mHealth applications}

Wearables and electrophysiological recordings disregard the subjective experience of motor symptoms and assessment of nonmotor symptoms. We believe that subjective experience of motor symptoms could improve the interpretation of objective motor symptom monitoring. Nonmotor symptoms are important for quality-of-life scores and might predict overall DBS outcomes ${ }^{64}$. Electronic health (eHealth) and mobile health (mHealth) applications and telemonitoring concepts have been recently integrated into PD care and contain the aforementioned missing features ${ }^{65-68}$. Most of these developments are achieved in order to improve PD care and ensure its accessibility and cost-effectiveness 69,70 . However, these developments also hold promise for aDBS.

A recent trial demonstrated that cDBS-setting adjustment by telemonitoring was feasible 71 introducing automated monitoring and increasing the frequency of DBSsetting adjustment brings this concept close to (semicontinuous) aDBS. The lack of valid continuous PD monitoring tools led to development of multimodal PD monitoring systems. These systems include, for example, wearables and mobile applications and distinguish themselves from systems discussed above by adding assessments of cognition, speech, subjective disease burden, and active motor tasks. This potential was recently underlined by development of a smartphone application to capture symptom fluctuation during the day ${ }^{72}$.

Several recently initiated trials test the feasibility and clinical value of multimodal PD monitoring systems in the patient's home environment. So far, these systems aim to differentiate ON/OFF states by wearables and a diary ${ }^{73}$, detect the need for changes in or improve adherence of pharmacological therapy ${ }^{74-76}$ and monitor clinical wellbeing in a holistic fashion ${ }^{77,78}$. Other systems aim to detect relevant neurophysiological biomarkers for home monitoring in order to improve postoperative DBS care67 and assess the effect of DBS parameter adjustments withwearables ${ }^{65}$. Furthermore, feasibility of the experience sampling method is demonstrated among PD patients ${ }^{79}$. This method collects subjective experiences of both motor and nonmotor symptoms multiple times a day during the flow of daily life.

The abovementioned studies show the feasibility of using multimodal monitoring systems among PD patients. We believe there might be a role for such multimodal PD monitoring systems in aDBS, because they have the potential to combine subjective assessments of burden and nonmotor symptoms with objective input signals. Especially during the initial 
postoperative phase, combining these input signals may be of great value for adjusting DBS. Feasibility of such a holistic approach should be explored further.

\section{Stimulation parameter modulation in aDBS}

The process of collecting continuous data representing (non-)motor symptoms is the first major challenge for developing an aDBS system for PD. A second major challenge is the design of a system that automates the complex reasoning and decision making currently achieved by clinicians, which will require more advanced and distinctive signal processing than is currently available. This challenge contains several issues, such as frequency of stimulation parameter adjustments, the nature of stimulation parameter adjustments, data transfer, data computation, and battery consumption.

Most aDBS research has so far focused on potential input signals, and therefore several issues regarding the design of stimulation parameter modulation are less well studied. In the following sections, we discuss the current progress and remaining challenges regarding stimulation parameter modulation in aDBS.

\section{Amplitude Modulation Approaches}

All reported aDBS systems in PD until now are based on automatic amplitude modulation (AM). AM can be applied in different designs. ON/OFF AM is an aDBS paradigm that varies between periods during which stimulation is given with a predefined amplitude and a set frequency and pulse width, and periods during which stimulation is switched off (Fig. 4A). ON/OFF AM systems studied in akinetic-rigid PD patients applied stimulation as long as the beta-LFP recorded in the STN exceeded a certain threshold ${ }^{5,28}$. In contrast, ON/OFF AM systems studied in tremor-dominant PD patients were designed to start a stimulation period several seconds before tremor reoccurs based on tremor prediction using machinelearning algorithms ${ }^{44,46}$. Because tremor should not reoccur during stimulation, there is no feedback signal that identifies the end of the stimulation period. Recent research has shown that a stimulation duration of 30 seconds led to a ratio of stimulation time versus tremor-free time off-stimulation over $50 \%$ in one third of patients ${ }^{61}$. Future research will need to clarify how ON/OFF AM can be implemented optimally for different PD phenotypes and different input signals.

When using ON/OFF AM, other details should be considered. First, a ramping onset, which increases the stimulation voltage from zero toward a predefined amplitude, can be used to overcome paresthesia ${ }^{5,32}$. Furthermore, ON/OFF AM can be applied in a phase dependent manner, in which a stimulus is applied with a fixed latency to an input signal ${ }^{25}$. Phase-dependent aDBS is hypothesized to have advantages over standard aDBS. Increased clinical benefit is suggested by targeting specific pathological neurophysiological phases in PD 5,32,34. Also, phase-dependent aDBS might induce long-lasting beneficial effects 
attributed to possible long-term potentiation/depotentiation in the STN ${ }^{80}$. Moreover, phase-dependent aDBS reduced tremor severity and prevented breakthrough tremor while consuming less energy compared to $\mathrm{CDBS}^{81}$. Studies assessing these suggested advantages of phase dependency in aDBS are required.

Other AM aDBS paradigms use a gradual or a continuous AM approach. Gradual AM increases or decreases the amplitude stepwise when the input signal is respectively higher or lower than certain thresholds (Fig. 4B) ${ }^{6,41}$. Minimal and maximal stimulation amplitudes and the voltage change per step have yet to be defined. Recently, a gradual AM approach based on tremor power introduced two feedback loop computations. One slow loop gradually adjusted the amplitude baseline to prevent re-emergence of diminished tremor, and one fast loop adjusted the actual amplitude rapidly to mitigate occurring tremor ${ }^{82}$. This design will need to be reproduced, and the added benefit should be assessed. Continuous AM links every possible input signal to a corresponding preset output amplitude (Fig. 4C). Thus, the output amplitude inclines toward a parallel line of the input signal ${ }^{21,22}$.

It is clear that stimulation parameter modulation can be applied in several ways in aDBS. At this moment, no research has been done to compare different approaches in general, or for specific phenotypes or input signals. In the next paragraph, we will elaborate on the clinical demands toward stimulation parameter modulation in aDBS per phenotype.

\section{Stimulation parameter modulation demands per phenotype}

An optimally performing aDBS system should prevent overstimulation during periods with less symptoms, and it should increase voltage in a timely manner to minimize the duration and severity of symptomatic periods. Therefore, frequency of input signal evaluation can be an important difference in stimulation parameter modulation according to phenotype. This should be based on the frequency at which the monitored symptom is expected to fluctuate or reoccur after stopping or decreasing stimulation.

In tremor-dominant PD, an aDBS system should ideally stimulate on "tremor control" level before the tremor actually occurs. Given that tremor fluctuates rapidly, the AM approach should rapidly respond to tremor reoccurrence in order to minimize tremor duration.

Compared to ON/OFF AM, a gradual or continuous AM approach needs more evaluation before stimulation reaches tremor-control level. However, ON/OFF AM always stimulates with the preset voltage, which might cause overstimulation. 


\section{A: ON/OFF}
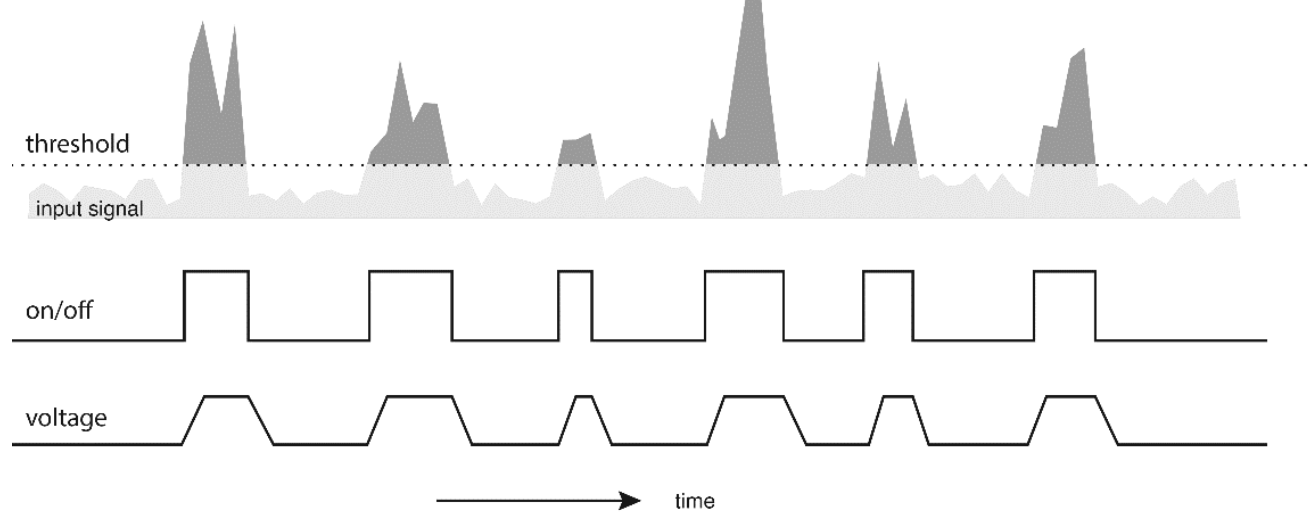

\section{B: gradual}
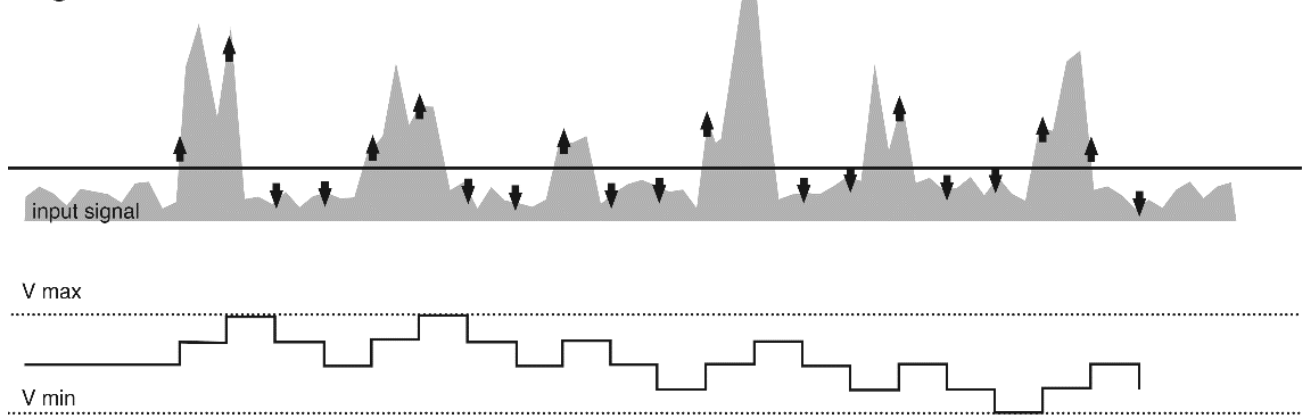

\section{C: continuous}

input signal

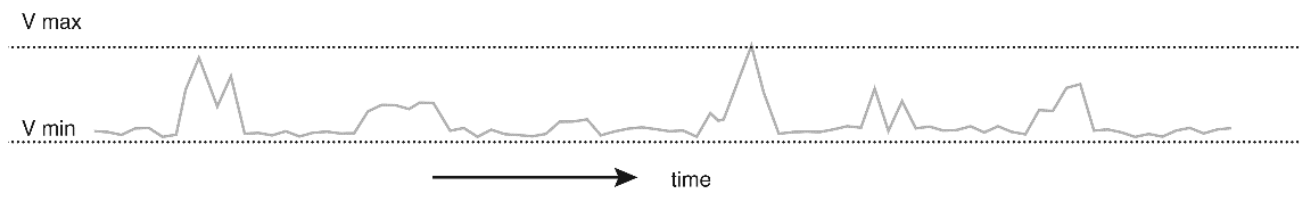

Figure 4. Schematic overview of different amplitude modulation paradigms used in aDBS in PD. (A) ON/OFF paradigm, which stimulates with ramping onset when input signals exceed a certain threshold. (B) Gradual paradigm, which increases or decreases stimulation amplitude stepwise when input signal exceeds or does not exceed a certain threshold respectively. (C) Continuous paradigm, which modifies stimulation amplitude according to strength of input signal. 
In developing an optimal AM approach, development of tremor prediction machinelearning models is important ${ }^{46}$. At the moment, most of these models are very accurate in scaling tremor severity rather than predicting reoccurrence ${ }^{83}$. Future studies should analyze different evaluation frequencies, corresponding computational costs, and tremor reduction to compare the feasibility of different $A M$ approaches. In akinetic-rigid PD, motor symptom fluctuations will be less frequent and less acute. Different input signal evaluation frequencies in aDBS for akinetic-rigid patients have not yet been compared. Whether gradual or continuous AM is superior to ON/OFF AM in this group is dependent on improved symptom reduction and prevention of over-stimulation when stimulating between zero and maximal amplitude.

Recent work on the modulatory effect of aDBS on beta-LFP suggests ON/OFF AM to be better suited for akinetic-rigid patients than gradual AM ${ }^{32}$. They found a correlation between longer beta-bursts (>0.6 seconds) and clinical impairment. Consequently, this implies that these longer beta bursts should trigger stimulation, and rapid anticipation and frequent evaluation of beta power is thus needed. Because of this required rapid anticipation, they prefer ON/OFF AM.

However, if the input signal follows the rhythm of akinesia and rigidity fluctuations, the input signal evaluation frequency could decrease, and a gradual AM approach might also be suitable and efficient. Whether this less-frequent evaluation is feasible with STN-LFP recordings has not been explored. At the moment, wearable sensors might have more potential to accomplish this compared to STN-LFP recordings.

In aDBS for PD patients suffering moderate dyskinesia, these considerations were also addressed ${ }^{41}$. The researchers observed aDBS transitions more frequently than expected based on clinical symptomatology. They suggested a slow ramping onset of stimulation voltage adjustments or alternative use of the triggering threshold, for example, a higher threshold or a two-step threshold, in order to prevent too frequent stimulation parameter adjustments.

\section{Future considerations}

\section{Issues for clinical implementation}

As discussed above, the research field on aDBS in PD is rapidly evolving (Fig. 1). In this section, we will highlight additional prospective issues that should be solved to realize a feasible aDBS system for chronic therapy.

Individual expectations and desires regarding an aDBS system can differ because of interindividual differences in the clinical course and personal coping strategies in PD patients. Individually tailored aDBS paradigms should respond to these factors, particularly aDBS systems that enable personal nuances in stimulation parameter modulation. 
However, aDBS in PD first needs a feasible standard system and stimulation parameter modulation, or one aDBS system per phenotype, before individualized fine-tuning can take place. It is plausible that each individual will start aDBS therapy with a calibration period, similar to CDBS therapy. Instead of a trial-and-error period trying different amplitudes, frequencies, or electrode contacts, the aDBS calibration period might try out different frequencies of stimulation parameter modulation, different threshold levels, or different voltage-steps per modulation. Ideally, this process is automated by a self-regulating algorithm.

\section{aDBS during sleep}

Although not discussed yet, a feasible aDBS system should consider the differences in patient preference and input signal during sleep. Akinetic-rigid patients might consider stimulation at night as important, given that they suffer from rigidity at night and in the morning. Tremor-dominant patients might need less stimulation at night because of a lower disease burden. Regarding input signals, electrophysiological signals are influenced by vigilance state and therefore deserve different interpretation during sleep periods than during awake periods ${ }^{31}$. Also, wearable sensors might be programmed with "sleep" or "rest" detection algorithms, which initiates a specific "sleep-stimulation paradigm."

\section{Monitoring of nonmotor symptoms and side effects}

In general, current aDBS input signals are focused on motor symptom detection to evaluate the therapeutic effect. As discussed before, a first step toward personalized therapy can be to develop different aDBS approaches for the different main motor symptoms per phenotype. Future designs might expand the specificity per phenotype by considering nonmotor symptoms ${ }^{84}$ and potentially side effects caused by aDBS, like autonomic functions, dyskinesia, or speech deterioration. Including these features will make data analysis even more complicated. This future challenge requires complicated nuances that are out of reach for aDBS presently.

\section{aDBS modulation other than amplitude modulation}

Later aDBS systems might explore the use of different stimulation parameter modulations for specific clinical situations, for example, frequency modulation (FM). Possibly, stimulation parameters in bilateral aDBS could be evaluated and adjusted per side separately, tailoring aDBS per side.

Application of FM is hypothesized to contribute to tailored DBS paradigms ${ }^{85}$. Three recent reviews on low-frequency STN-DBS described beneficial effects on freezing of gait, speech, and swallowing that did not respond to, or were caused by, high-frequency DBS. However, beneficial effects could not always be reproduced, and low-frequency stimulation sometimes led to worsening of cardinal PD symptoms ${ }^{86-88}$. The effect of variable frequency 32 
stimulation, a paradigm interleaving high- and low-frequency DBS ${ }^{89}$, will be explored soon 90.

Also, pulse-width modulation might provide clinical benefit in certain situations. By exciting thin axon bundles belonging to the direct cortico-subthalamic pathway more selectively ${ }^{91}$, therapeutic windows may increase using shorter pulse widths, while using less energy ${ }^{92,93}$.

\section{Battery power balance}

aDBS may require less battery power for stimulation compared to cDBS. In contrast, more battery power may be needed for data processing and transferal, for example, by Bluetooth. There are several options to minimize the additional power needed by the pulse generator and to eventually make battery replacement less frequent. First, comparing the computational demands of various signal processing and machine-learning approaches should minimize the required power ${ }^{16}$. Second, the possibility to perform analyses on external devices or cloud-platform solutions should be evaluated. Energy saved by outsourcing these computations should be compared with the energy required of wireless data transfer. Third, rechargeable pulse generators should be further developed regarding clinical applicability ${ }^{94}$.

\section{Socioeconomical relevance}

We suggest that implementation of aDBS systems in PD care will result in beneficial socioeconomic effects. Most important, if aDBS results in an improved ratio between beneficial and side effects, quality of life will improve and patients will function better in society. Also, economic burden will decrease, given that PD patients can be part of the working population for a longer period and need less care.

\section{Conclusion}

Although impressive progress in aDBS for PD has been made over the last decade, major challenges to chronic application are still pending. We believe that research into clinical associations of input signals should concentrate on different PD phenotypes. Because the correlation of different input signals with PD symptomatology varies (Fig. 3), we believe that no single currently available input signal will cover the heterogeneity of all phenotypes in PD patients. To achieve this ambition, thoughtful combining and selection of input signals is inevitable. The increasing trend of combining knowledge between neurologists, neurosurgeons, engineers, and computer scientists is crucial in this field and opens the gate to translational medicine 2.0: "from byte to bedside." 


\section{References}

1. Janssen, M. L. F. et al. Subthalamic nucleus high-frequency stimulation for advanced Parkinson's disease: motor and neuropsychological outcome after 10 years. Stereotact Funct Neurosurg 92, 381387 (2014).

2. Limousin, P. et al. Effect of parkinsonian signs and symptoms of bilateral subthalamic nucleus stimulation. Lancet 345, 91-95 (1995).

3. Tripoliti, E. et al. Effects of subthalamic stimulation on speech of consecutive patients with Parkinson disease - PubMed. Neurology (2011) doi:10.1212/WNL.0b013e318203e7d0.

4. Deuschl, G. et al. A randomized trial of deep-brain stimulation for Parkinson's disease. N Engl J Med 355, 896-908 (2006).

5. Little, S. et al. Adaptive deep brain stimulation in advanced Parkinson disease. Ann Neurol 74, 449-457 (2013).

6. Malekmohammadi, M. et al. Kinematic Adaptive Deep Brain Stimulation for Resting Tremor in Parkinson's Disease. Mov Disord 31, 426-428 (2016).

7. Arlotti, M., Rosa, M., Marceglia, S., Barbieri, S. \& Priori, A. The adaptive deep brain stimulation challenge. Parkinsonism Relat Disord 28, 12-17 (2016).

8. Giannicola, G. et al. The effects of levodopa and ongoing deep brain stimulation on subthalamic beta oscillations in Parkinson's disease. Exp Neurol 226, 120-127 (2010).

9. Little, S., Pogosyan, A., Kuhn, A. A. \& Brown, P. $\beta$ band stability over time correlates with Parkinsonian rigidity and bradykinesia. Exp Neurol 236, 383-388 (2012).

10. Beudel, M. et al. Oscillatory Beta Power Correlates With Akinesia-Rigidity in the Parkinsonian Subthalamic Nucleus. Mov Disord 32, 174-175 (2017).

11. Chen, C. C. et al. Complexity of subthalamic $13-35 \mathrm{~Hz}$ oscillatory activity directly correlates with clinical impairment in patients with Parkinson's disease. Exp Neurol 224, 234-240 (2010).

12. Kühn, A. A., Kupsch, A., Schneider, G.-H. \& Brown, P. Reduction in subthalamic 8-35 Hz oscillatory activity correlates with clinical improvement in Parkinson's disease. Eur J Neurosci 23, 1956-1960 (2006).

13. Contarino, M. F. et al. Tremor-specific neuronal oscillation pattern in dorsal subthalamic nucleus of parkinsonian patients. Brain Stimul 5, 305-314 (2012).

14. Hirschmann, J., Schoffelen, J. M., Schnitzler, A. \& van Gerven, M. a. J. Parkinsonian rest tremor can be detected accurately based on neuronal oscillations recorded from the subthalamic nucleus. Clin Neurophysiol 128, 2029-2036 (2017).

15. Syrkin-Nikolau, J. et al. Subthalamic neural entropy is a feature of freezing of gait in freely moving people with Parkinson's disease. Neurobiol Dis 108, 288-297 (2017).

16. Golshan, H. M., Hebb, A. O., Hanrahan, S. J., Nedrud, J. \& Mahoor, M. H. A hierarchical structure for human behavior classification using STN local field potentials. J Neurosci Methods 293, 254-263 (2018).

17. Neumann, W.-J. et al. Deep Brain Recordings Using an Implanted Pulse Generator in Parkinson's Disease. Neuromodulation 19, 20-24 (2016).

18. Little, S. et al. Bilateral adaptive deep brain stimulation is effective in Parkinson's disease. J Neurol Neurosurg Psychiatry 87, 717-721 (2016).

19. Little, S. et al. Adaptive deep brain stimulation for Parkinson's disease demonstrates reduced speech side effects compared to conventional stimulation in the acute setting. J Neurol Neurosurg Psychiatry 87, 1388-1389 (2016).

20. Rosa, M. et al. Adaptive deep brain stimulation controls levodopa-induced side effects in Parkinsonian patients. Mov Disord 32, 628-629 (2017)

21. Arlotti, M. et al. Eight-hours adaptive deep brain stimulation in patients with Parkinson disease. Neurology 90, e971-e976 (2018).

22. Rosa, M. et al. Adaptive deep brain stimulation in a freely moving Parkinsonian patient. Mov Disord 30, 1003-1005 (2015).

23. Piña-Fuentes, D. et al. Adaptive DBS in a Parkinson's patient with chronically implanted DBS: A proof of principle. Mov Disord 32, 1253-1254 (2017).

24. Shreve, L. A. et al. Subthalamic oscillations and phase amplitude coupling are greater in the more affected hemisphere in Parkinson's disease. Clin Neurophysiol 128, 128-137 (2017). 
25. Meidahl, A. C. et al. Adaptive Deep Brain Stimulation for Movement Disorders: The Long Road to Clinical Therapy. Mov Disord 32, 810-819 (2017).

26. Blumenfeld, Z. et al. Sixty-hertz stimulation improves bradykinesia and amplifies subthalamic lowfrequency oscillations. Mov Disord 32, 80-88 (2017).

27. van Wijk, B. C. M. et al. Subthalamic nucleus phase-amplitude coupling correlates with motor impairment in Parkinson's disease. Clin Neurophysiol 127, 2010-2019 (2016).

28. Johnson, L. A. et al. Closed-Loop Deep Brain Stimulation Effects on Parkinsonian Motor Symptoms in a Non-Human Primate - Is Beta Enough? Brain Stimul 9, 892-896 (2016).

29. Storzer, L. et al. Bicycling suppresses abnormal beta synchrony in the Parkinsonian basal ganglia. Ann Neurol 82, 592-601 (2017).

30. Hell, F., Taylor, P. C. J., Mehrkens, J. H. \& Bötzel, K. Subthalamic stimulation, oscillatory activity and connectivity reveal functional role of STN and network mechanisms during decision making under conflict. Neuroimage 171, 222-233 (2018).

31. Escobar Sanabria, D. et al. Parkinsonism and vigilance: alteration in neural oscillatory activity and phase-amplitude coupling in the basal ganglia and motor cortex. J Neurophysiol 118, 2654-2669 (2017).

32. Tinkhauser, G. et al. The modulatory effect of adaptive deep brain stimulation on beta bursts in Parkinson's disease. Brain 140, 1053-1067 (2017).

33. Hammond, C., Bergman, H. \& Brown, P. Pathological synchronization in Parkinson's disease: networks, models and treatments. Trends Neurosci 30, 357-364 (2007).

34. Rosin, B. et al. Closed-loop deep brain stimulation is superior in ameliorating parkinsonism. Neuron 72 , 370-384 (2011).

35. de Hemptinne, C. et al. Exaggerated phase-amplitude coupling in the primary motor cortex in Parkinson disease. Proc Natl Acad Sci U S A 110, 4780-4785 (2013).

36. de Hemptinne, $C$. et al. Therapeutic deep brain stimulation reduces cortical phase-amplitude coupling in Parkinson's disease. Nat Neurosci 18, 779-786 (2015).

37. Qasim, S. E. et al. Electrocorticography reveals beta desynchronization in the basal ganglia-cortical loop during rest tremor in Parkinson's disease. Neurobiol Dis 86, 177-186 (2016).

38. Swann, N. C. et al. Gamma Oscillations in the Hyperkinetic State Detected with Chronic Human Brain Recordings in Parkinson's Disease. J Neurosci 36, 6445-6458 (2016).

39. McCrimmon, C. M. et al. Electrocorticographic Encoding of Human Gait in the Leg Primary Motor Cortex. Cereb Cortex 28, 2752-2762 (2018).

40. Herff, C. \& Schultz, T. Automatic Speech Recognition from Neural Signals: A Focused Review. Front Neurosci 10, 429 (2016).

41. Swann, N. C. et al. Adaptive deep brain stimulation for Parkinson's disease using motor cortex sensing. J Neural Eng 15, 046006 (2018).

42. Cole, S. R. et al. Nonsinusoidal Beta Oscillations Reflect Cortical Pathophysiology in Parkinson's Disease. J Neurosci 37, 4830-4840 (2017).

43. Pittman-Polletta, B., Hsieh, W.-H., Kaur, S., Lo, M.-T. \& Hu, K. Detecting phase-amplitude coupling with high frequency resolution using adaptive decompositions. J Neurosci Methods 226, 15-32 (2014).

44. Basu, I. et al. Pathological tremor prediction using surface electromyogram and acceleration: potential use in 'ON-OFF' demand driven deep brain stimulator design. J Neural Eng 10, 036019 (2013).

45. Camara, C. et al. A Fuzzy Inference System for Closed-Loop Deep Brain Stimulation in Parkinson's Disease. J Med Syst 39, 155 (2015).

46. Khobragade, N., Graupe, D. \& Tuninetti, D. Towards fully automated closed-loop Deep Brain Stimulation in Parkinson's disease patients: A LAMSTAR-based tremor predictor. Annu Int Conf IEEE Eng Med Biol Soc 2015, 2616-2619 (2015).

47. Scholz, E. et al. Medium and long latency EMG responses in leg muscles: Parkinson's disease. J Neurol Neurosurg Psychiatry 50, 66-70 (1987).

48. Graupe, D., Basu, I., Tuninetti, D., Vannemreddy, P. \& Slavin, K. V. Adaptively controlling deep brain stimulation in essential tremor patient via surface electromyography. Neurol Res 32, 899-904 (2010).

49. Herron, J. A. et al. Chronic electrocorticography for sensing movement intention and closed-loop deep brain stimulation with wearable sensors in an essential tremor patient. J Neurosurg 127, 580-587 (2017).

50. Yamamoto, T. et al. On-demand control system for deep brain stimulation for treatment of intention tremor - PubMed. Neuromodulation (2013) doi:10.1111/j.1525-1403.2012.00521.x. 
51. Askari, S., Zhang, M. \& Won, D. S. An EMG-based system for continuous monitoring of clinical efficacy of Parkinson's disease treatments. Annu Int Conf IEEE Eng Med Biol Soc 2010, 98-101 (2010).

52. Levin, J. et al. Objective measurement of muscle rigidity in Parkinsonian patients treated with subthalamic stimulation. Mov Disord 24, 57-63 (2009).

53. Chang, S.-Y. et al. Development of the Mayo Investigational Neuromodulation Control System: toward a closed-loop electrochemical feedback system for deep brain stimulation. J Neurosurg 119, 1556 1565 (2013).

54. Grahn, P. J. et al. A neurochemical closed-loop controller for deep brain stimulation: toward individualized smart neuromodulation therapies. Front Neurosci 8, 169 (2014).

55. Sánchez-Ferro, Á. et al. New methods for the assessment of Parkinson's disease (2005 to 2015): A systematic review. Mov Disord 31, 1283-1292 (2016).

56. Delrobaei, M. et al. Towards remote monitoring of Parkinson's disease tremor using wearable motion capture systems. J Neurol Sci 384, 38-45 (2018).

57. Rodríguez-Martín, D. et al. Home detection of freezing of gait using support vector machines through a single waist-worn triaxial accelerometer. PLoS One 12, e0171764 (2017).

58. Delrobaei, M., Baktash, N., Gilmore, G., Mclsaac, K. \& Jog, M. Using Wearable Technology to Generate Objective Parkinson's Disease Dyskinesia Severity Score: Possibilities for Home Monitoring. IEEE Trans Neural Syst Rehabil Eng 25, 1853-1863 (2017).

59. Griffiths, R. I. et al. Automated assessment of bradykinesia and dyskinesia in Parkinson's disease. J Parkinsons Dis 2, 47-55 (2012).

60. Hasan, H., Athauda, D., Foltynie, T. \& Noyce, A. Technologies Assessing Limb Bradykinesia in Parkinson's Disease - PubMed. J Parkinsons Dis (2017) doi:10.3233/JPD-160878.

61. Graupe, D. et al. Who May Benefit From On-Demand Control of Deep Brain Stimulation? Noninvasive Evaluation of Parkinson Patients. Neuromodulation 21, 611-616 (2018).

62. Kubota, K. J., Chen, J. A. \& Little, M. A. Machine learning for large-scale wearable sensor data in Parkinson's disease: Concepts, promises, pitfalls, and futures. Mov Disord 31, 1314-1326 (2016).

63. van Uem, J. M. T. et al. A Viewpoint on Wearable Technology-Enabled Measurement of Wellbeing and Health-Related Quality of Life in Parkinson's Disease. J Parkinsons Dis 6, 279-287 (2016).

64. Dafsari, H. S. et al. Short-term quality of life after subthalamic stimulation depends on non-motor symptoms in Parkinson's disease. Brain Stimul 11, 867-874 (2018).

65. Angeles, P., Tai, Y., Pavese, N., Wilson, S. \& Vaidyanathan, R. Automated assessment of symptom severity changes during deep brain stimulation (DBS) therapy for Parkinson's disease. IEEE Int Conf Rehabil Robot 2017, 1512-1517 (2017).

66. Eberle, W., Penders, J. \& Yazicioglu, R. F. Closing the loop for Deep Brain Stimulation implants enables personalized healthcare for Parkinson's disease patients. Annu Int Conf IEEE Eng Med Biol Soc 2011, 1556-1558 (2011).

67. Marceglia, S. et al. Web-based telemonitoring and delivery of caregiver support for patients with Parkinson disease after deep brain stimulation: protocol. JMIR Res Protoc 4, e30 (2015).

68. Zhang, C. et al. A Remote and Wireless Deep Brain Stimulation Programming System. Neuromodulation 19, 437-439 (2016).

69. Dorsey, E. R. et al. Moving Parkinson care to the home. Mov Disord 31, 1258-1262 (2016).

70. Schneider, R. B. \& Biglan, K. M. The promise of telemedicine for chronic neurological disorders: the example of Parkinson's disease. Lancet Neurol 16, 541-551 (2017).

71. Li, D. et al. Remotely Programmed Deep Brain Stimulation of the Bilateral Subthalamic Nucleus for the Treatment of Primary Parkinson Disease: A Randomized Controlled Trial Investigating the Safety and Efficacy of a Novel Deep Brain Stimulation System. Stereotact Funct Neurosurg 95, 174-182 (2017).

72. Zhan, A. et al. Using Smartphones and Machine Learning to Quantify Parkinson Disease Severity: The Mobile Parkinson Disease Score. JAMA Neurol 75, 876-880 (2018).

73. Bayés, À. et al. A 'HOLTER' for Parkinson's disease: Validation of the ability to detect on-off states using the REMPARK system. Gait Posture 59, 1-6 (2018).

74. Ferreira, J. J. et al. Quantitative home-based assessment of Parkinson's symptoms: the SENSE-PARK feasibility and usability study. BMC Neurol 15, 89 (2015).

75. Lakshminarayana, R. et al. Smartphone- and internet-assisted self-management and adherence tools to manage Parkinson's disease (SMART-PD): study protocol for a randomised controlled trial (v7; 15 August 2014). Trials 15, 374 (2014).

76. Tzallas, A. T. et al. PERFORM: a system for monitoring, assessment and management of patients with Parkinson's disease. Sensors (Basel) 14, 21329-21357 (2014). 
77. Cancela, J. et al. Monitoring of motor and non-motor symptoms of Parkinson's disease through a mHealth platform. Annu Int Conf IEEE Eng Med Biol Soc 2016, 663-666 (2016).

78. Earhart, A. ProViewTM. Kinesia http://gIneurotech.com/kinesia/products/proview/ (2014).

79. Broen, M. P. G. et al. Unraveling the Relationship between Motor Symptoms, Affective States and Contextual Factors in Parkinson's Disease: A Feasibility Study of the Experience Sampling Method. PLoS One 11, e0151195 (2016).

80. Azodi-Avval, R. \& Gharabaghi, A. Phase-dependent modulation as a novel approach for therapeutic brain stimulation. Front Comput Neurosci 9, 26 (2015).

81. Cagnan, H. et al. Stimulating at the right time: phase-specific deep brain stimulation. Brain 140, 132145 (2017).

82. Herron, J., Velisar, A., Malekmohammadi, M., Bronte-Stewart, H. \& Chizeck, H. J. A Metric for Evaluating and Comparing Closed-Loop Deep Brain Stimulation Algorithms. arXiv:1605.09312 [q-bio] (2016)

83. Jeon, $\mathrm{H}$. et al. High-accuracy automatic classification of Parkinsonian tremor severity using machine learning method. Physiol Meas 38, 1980-1999 (2017).

84. Marras, C. \& Chaudhuri, K. R. Nonmotor features of Parkinson's disease subtypes. Mov Disord 31, 1095-1102 (2016).

85. Fasano, A. \& Lozano, A. M. The FM/AM world is shaping the future of deep brain stimulation. Mov Disord 29, 161-163 (2014).

86. Baizabal-Carvallo, J. F. \& Alonso-Juarez, M. Low-frequency deep brain stimulation for movement disorders. Parkinsonism Relat Disord 31, 14-22 (2016).

87. Dayal, V., Limousin, P. \& Foltynie, T. Subthalamic Nucleus Deep Brain Stimulation in Parkinson's Disease: The Effect of Varying Stimulation Parameters. J Parkinsons Dis 7, 235-245 (2017).

88. Xie, T. et al. Effect of low versus high frequency stimulation on freezing of gait and other axial symptoms in Parkinson patients with bilateral STN DBS: a mini-review. Transl Neurodegener 6, 13 (2017).

89. Jia, F. et al. Variable frequency stimulation of subthalamic nucleus for freezing of gait in Parkinson's disease. Parkinsonism Relat Disord 21, 1471-1472 (2015).

90. Jia, F. et al. Variable frequency stimulation of subthalamic nucleus in Parkinson's disease: Rationale and hypothesis. Parkinsonism Relat Disord 39, 27-30 (2017).

91. Reich, M. M. et al. Short pulse width widens the therapeutic window of subthalamic neurostimulation. Ann Clin Transl Neurol 2, 427-432 (2015).

92. Akbar, U. et al. Randomized, Blinded Pilot Testing of Nonconventional Stimulation Patterns and Shapes in Parkinson's Disease and Essential Tremor: Evidence for Further Evaluating Narrow and Biphasic $\mathrm{P}$ ulses. Neuromodulation 19, 343-356 (2016).

93. Steigerwald, F. et al. Pulse duration settings in subthalamic stimulation for Parkinson's disease. Mov Disord 33, 165-169 (2018).

94. Rizzi, M. et al. Internal Pulse Generators in Deep Brain Stimulation: Rechargeable or Not? World Neurosurg 84, 1020-1029 (2015). 



\section{Chapter 3}

\section{Monitoring Parkinson's disease symptoms during daily life: a feasibility study}

M. Heijmans, J.G.V. Habets, C. Herff, J. Aarts, A. Stevens, M.L. Kuijf, P.L. Kubben

Published. Nature Partner Journal Parkinson's Disease 2019; 5:21 


\begin{abstract}
Parkinson's disease symptoms are most often charted using the MDS-UPDRS. Limitations of this approach include the subjective character of the assessments and a discrepant performance in the clinic compared to the home situation. Continuous monitoring using wearable devices is believed to eventually replace this golden standard, but measurements often lack a parallel ground truth or are only tested in lab settings. To overcome these limitations, this study explores the feasibility of a newly developed Parkinson's disease monitoring system, which aims to measure Parkinson's disease symptoms during daily life by combining wearable sensors with an experience sampling method application. Twenty patients with idiopathic Parkinson's disease participated in this study. During a period of two consecutive weeks, participants had to wear three wearable sensors and had to complete questionnaires at seven semi-random moments per day on their mobile phone. Wearable sensors collected objective movement data, and the questionnaires containing questions about amongst others Parkinson's disease symptoms served as parallel ground truth. Results showed that participants wore the wearable sensors during $94 \%$ of the instructed timeframe and even beyond. Furthermore, questionnaire completion rates were high $(79,1 \%)$ and participants evaluated the monitoring system positively. A preliminary analysis showed that sensor data could reliably predict subjectively reported OFF moments. These results show that our Parkinson's disease monitoring system is a feasible method to use in a diverse Parkinson's disease population for at least a period of two weeks. For longer use, the monitoring system may be too intense and wearing comfort needs to be optimized.
\end{abstract}




\section{Introduction}

Parkinson's disease (PD) is a neurodegenerative disorder characterized by motor symptoms like tremor, rigidity and bradykinesia, which can fluctuate during the day. Nowadays, symptoms and disease course are charted by a clinician and are measured most often using the MDS-UPDRS for research purposes ${ }^{1}$. This is however associated with limitations: (1) symptoms and disease course are assessed with low frequency and therefore only permit a snapshot of the clinical situation and may include recall bias; (2) scores given by the clinician have a subjective character; (3) patients might put themselves in a better light during in-clinical assessments compared to when they are at home. In order to get more frequent and more objective ratings of symptoms and disease course, continuous monitoring systems are essential. Such systems are believed to represent the clinical symptoms in the daily life of patients more reliably and could eventually even be used as input signal for adaptive, responsive, or closed-loop deep brain stimulation (DBS) 2 .

Wearable sensors are increasingly used to detect PD symptoms due to technological innovations, resulting in small, low cost, power efficient, and accurate sensors ${ }^{3-5}$. Wearable sensors have shown promise in detecting tremor ${ }^{6-8}$, freezing of gait 9 , bradykinesia ${ }^{10}$, and dyskinesia ${ }^{10,11}$. Recently, various monitoring systems have been developed and tested and have shown promising results ${ }^{12-14}$. These studies made use of predefined motor tasks in a lab or simulated home setting, which only gives a limited representation of the daily life environment. Furthermore, these regular clinical assessments cannot be performed continuously in a daily life environment.

The newly developed PD monitoring system presented in this paper combines wearable sensors measuring acceleration and rotational acceleration with an experience sampling method (ESM) application (Fig. 1). ESM is a validated, digital diary method consisting of multiple repeated measurements at semi-random moments in daily life ${ }^{15,16}$. It is superior compared to standard diary and cross-sectional assessments, because there is no recall bias and data are collected on multiple moments a day ${ }^{17}$. The designed ESM questionnaires include questions regarding mood states, contextual information and both motor and nonmotor PD symptoms. ESM data may serve as a parallel ground truth to the wearable sensor data, making the impossible regular clinical assessments in daily life measurements redundant. The unique system combines objective data (wearable sensors) with subjective data (ESM) in the daily life of the patients.

Although the combination of wearable sensors with ESM or other electronic diary methods was often suggested in PD ${ }^{18,19}$, and although this combination was used in other populations before $20-25$, it has never been tested in PD so far. Consequently, this project aims to prove the feasibility of the new monitoring system in PD patients during daily life 
for two consecutive weeks. In addition, we investigated whether the ESM answers can be employed as a ground truth for the sensor data by performing an OFF moment prediction analysis. Eventually, we would like to further assess whether this system could also be used for closed-loop DBS programming.

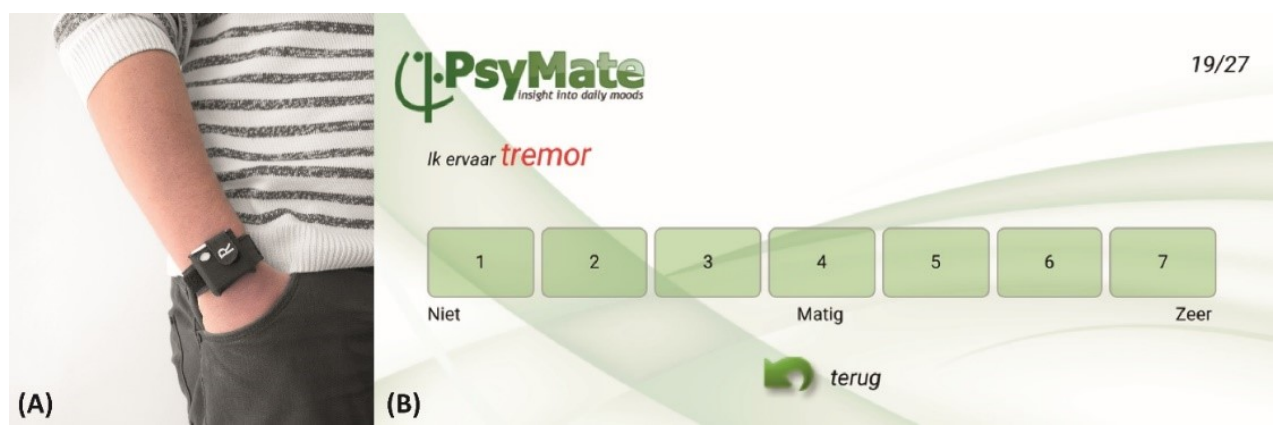

Figure 1. Recording modalities. a Wearable sensor attached to the wrist via a wristband. b Screenshot of the question 'Ik ervaar tremor' ('I experience tremor') on a 7 point Likert scale, with a score of 1 indicating not at all and a score of 7 indicating very much.

\section{Results}

\section{Wearable sensors}

On average, the group participants wore the wearable sensors $898 \mathrm{~min}$ a day, equalling almost $15 \mathrm{~h}$. The mean time that the wearable sensors were used by the participants was $788 \mathrm{~min}(94 \%)$ within the instructed timeframe of $8.00-22.00 \mathrm{~h}$ (Fig. 2). Consequently, the mean non-worn time within the instructed timeframe was $52 \mathrm{~min}(6 \%)$. The mean time that the wearable sensors were used by the participants outside the instructed timeframe was $110 \mathrm{~min}$.

Answers from the evaluation questionnaire showed that most participants found that the wrist $(60 \%)$ and the chest $(80 \%)$ sensors were comfortable to wear. However, some participants mentioned that the used accessories remained attached to clothes, or that they caused irritation. Two participants (10\%) reported that the wrist sensors impaired their arm movement. According to all participants, the wrist sensors did not impair hand movement nor did the three sensors impair movements in general. None of the participants found the sensors were heavy to wear, had problems with putting the sensors on and off, or had problems with charging the sensors. Almost all participants (95\%) wore the sensors in public. Some participants mentioned that they thought it was awkward to get questions about the sensors, and therefore they were glad that it was possible to wear the sensors beneath their clothes. Only 30 and $50 \%$ of the participants was willing to wear the wrist and chest sensors on a long-term basis. 


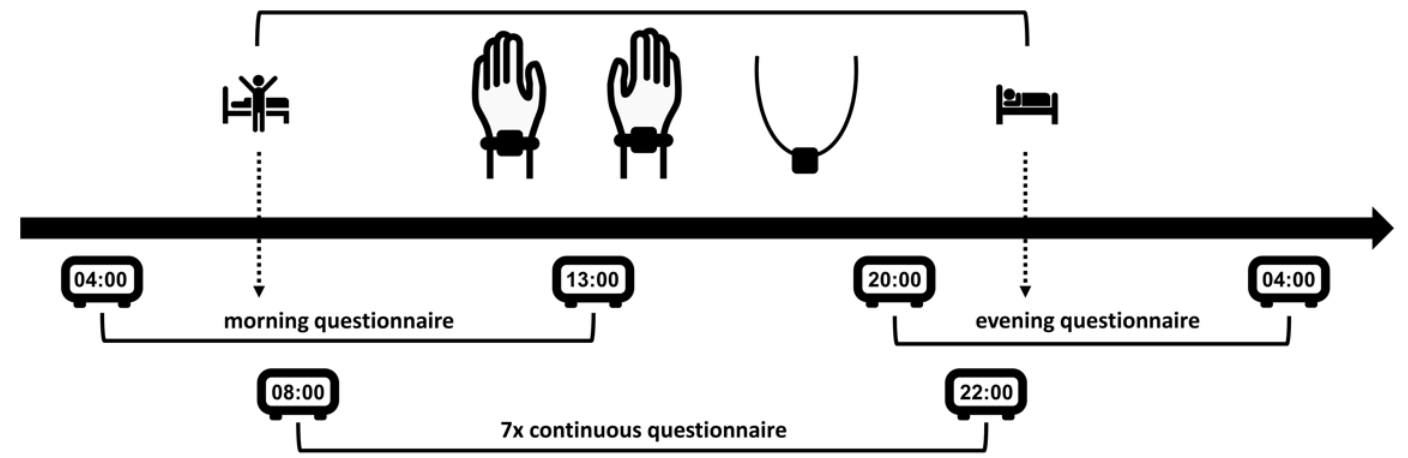

Figure 2. Schematic overview of one test day. Wearable sensors were worn from waking up until going to bed. Questionnaires were available (morning and evening) or showed up (continuous) between the indicated timeframes.

\section{Experience sampling method}

ESM is a validated, digital diary method consisting of multiple repeated measurements at semi-random moments. Participants received a total of 98 continuous questionnaires, 14 morning questionnaires, and 14 evening questionnaires (Fig. 2). Only fully completed questionnaires were considered as completed. On average, 77.5 continuous questionnaires, 13.6 morning questionnaires, and 13.2 evening questionnaires were completed. This resulted in completion rates of $79.1 \%$ for the continuous questionnaires, $96.8 \%$ for the morning questionnaires and $93.9 \%$ for the evening questionnaires. For each completed continuous questionnaire, it was checked whether there was sensor data available from the two wrist sensors and the chest sensor for at least 15 min before the questionnaire was opened by the participant. We hypothesize that this timeframe will best reflect the patients clinical state belonging to the corresponding ESM answer, and therefore this timeframe will be used for further analyses. In total, a mean of 69.0 (89.0\%) continuous questionnaires were completed which had corresponding data from all three sensors (Fig. 3). For the majority of participants, only a few continuous questionnaires did not have corresponding sensor data from all three sensors for at least 15 min preceding the questionnaire (Fig. 3). Three participants (7, 10, 14 in Fig. 3) missed sensor data for more than $25 \%$ of the completed questionnaires. This was because sensors stopped recording due to full data storage as a result of hidden folders containing the removed data of previous participants. Four other participants had some aborted recordings for still unknown reasons.

Answers from the evaluation questionnaire showed that all participants found the ESM application easy to use and answered the questions independently. The majority of the participants found the ESM questions clear (85\%). Some participants mentioned that the line of questioning was inconsistent. For example, a score of 1 was sometimes the most 
positive option and sometimes the most negative option. Three participants (15\%) found it unpleasant to carry their phone with them all day, for example because they could not due to work. Further, 17 participants (85\%) did not mind that the continuous questionnaires showed up at random moments during the day, which was supported by the average score of only 1.7 on the question 'I found this beep disturbing'. This item was scored on a 7 point Likert scale, where 1 corresponded with 'not disturbing at all' and 7 corresponded with 'very disturbing'. Three participants (15\%) thought they missed a lot of questionnaires and ten participants (50\%) said they would use the ESM application for a longer period than two consecutive weeks.

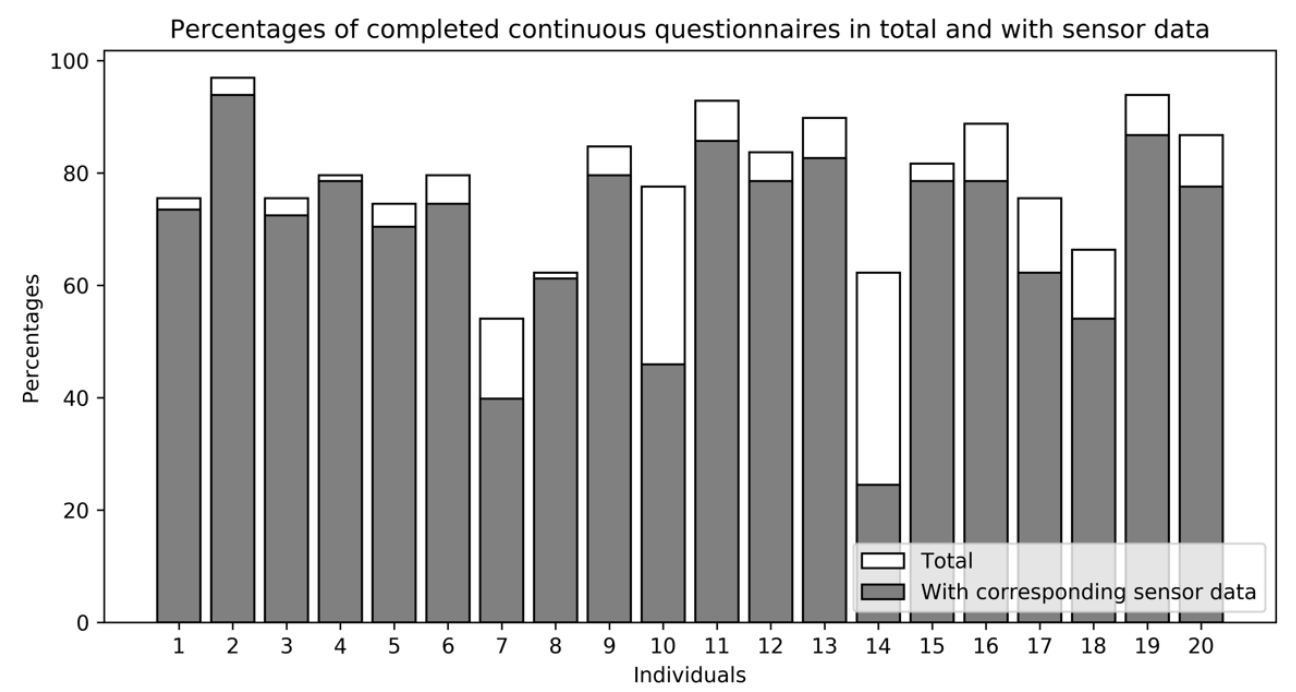

Figure 3. Completion rates. Percentages of total completed continuous questionnaires per participant (white bars) and completed continuous questionnaires with corresponding sensor data (grey bars).

\section{The monitoring system in general}

All participants reported that the information about the research was clear, and $95 \%$ found the aim of the research clear as well. Three participants (15\%) considered the study to be incriminating, and two participants (10\%) adapted their daily life because of the study. For example, one participant made shorter cycling trips, since this participant was afraid to miss questionnaires.

\section{Combining ESM and wearable sensor data}

Our pilot investigation on one patient with severe ON/OFF fluctuations yielded a reliable detection of subjectively registered OFF moments based on sensor data using a logistic regression classifier (area under the curve $=0.73$ ). Figure 4 shows true positive and false 
positive rates for different thresholds (Receiver-Operator-Characteristic). These initial results highlight the feasibility of using wearable sensors to detect symptom severity.

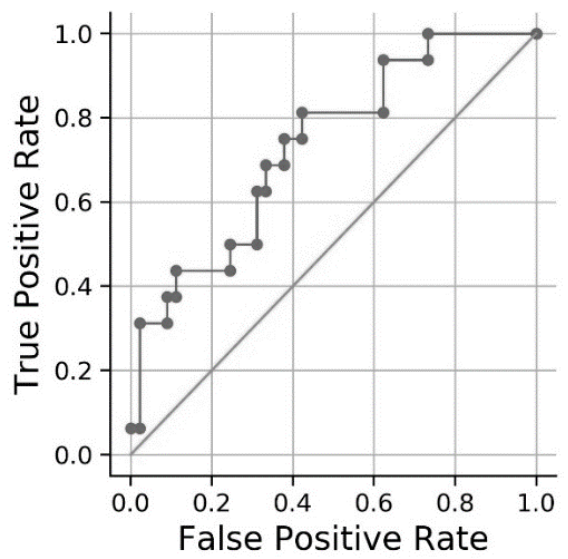

Figure 4. Receiver-Operator-Characteristic curve of detection of OFF moments from sensor data. Aurea under de curve $=0.73$.

\section{Discussion}

This study showed that combining wearable sensors with ESM is a feasible method for monitoring PD patients in daily life. Results showed that participants wore the wearable sensors almost during the whole instructed timeframe and even beyond, showing that the wearable sensors were not obstructive. Furthermore, ESM completion rates were high and participants evaluated the monitoring system positively. The presented participant characteristics (Table 1) suggest that this monitoring system is feasible for a diverse population of PD patients. For example, age was ranging between 46 and 74 years, disease duration was ranging between 1 and 21 years, and no restrictions were made based on PD phenotype and treatment. There was a relatively high proportion of patients with DBS implants. This selection was most probably due to the population of the academic hospital. We cannot rule out the possibility that PD patients with DBS are more willingly to use technological devices such as this monitoring system.

ESM completion rates were high, with an average of $79 \%$. All participants met the requirement defined in previous work, which demands that at least one third of the continuous questionnaires should be completed to have valid ESM data ${ }^{26}$. The high completion rate may be due to the extensive briefing during the start session and due to the phone contact moments with the participants on day 2 and 8 . Compared to previous work using the same ESM application for 5 days in 5 PD patients, our completion rates were $5 \%$ lower ${ }^{27}$. One might argue that the longer use of the ESM application in this study might explain lower completion rates. This argument is supported by a previous $\mathrm{N}=1$ study, in 
which the completion rate was $47 \%$ in week 1 and dropped to $29 \%$ in week $2{ }^{18}$. We did however not see any differences between average completion rates per day (Supplementary Fig. 1). Also, there were no differences in completion rates between timeframes (Supplementary Fig. 2). The high completion rates in general are illustrated by the fact that all patients found the application easy to use, and that even half of the patients is willing to use the ESM application for a longer period.

Two participants reported they adapted their daily life during the study period. This was because participants were for example afraid to miss questionnaires. We believe that by using ESM, participants might indeed be more 'on guard' and they are required to carry their mobile phone all day. For non-digital questionnaires, there is to our knowledge no study describing whether participants adapted their daily life during the study period. We hypothesize that this will also be the case, since nondigital questionnaires mostly have to be completed at set times. Regarding completion rates it is hard to compare ESM with nondigital diary methods since non-digital diaries can be completed at different moments than instructed.

The evaluation questionnaire outcomes showed that there is room for improvement regarding the used materials of the wearable sensor accessories. The accessories used in this study were developed with the aim that they should be easy to handle for PD patients. This was the case, since none of the participants had trouble with putting the sensors on or off. However, the use of Velcro resulted in the disadvantage that the sensors sometimes remained attached to clothes. Another disadvantage was that the sensors and accessories were not attractive enough to wear. Improvement of the accessories will likely result in a higher amount of patients who find the wearable sensors comfortable to wear and who would wear the wearable sensors on a chronic basis.

The next steps of this study are to validate ESM data, improve PD symptom algorithms for wearable data, correlate wearable data with ESM data, and eventually predict ESM scores based on wearable data. Since the combination of wearable data with ESM data is new in itself, we propose the following data processing steps: for each completed continuous ESM questionnaire, 15 minutes of sensor data prior to this completed questionnaire will be extracted. We hypothesize that this timeframe will best reflect the patients clinical state belonging to the corresponding ESM answer. It should however be tested which timeframe best fits with the questionnaire timestamp. This might be much shorter or longer than the proposed $15 \mathrm{~min}$, or might even be after completion of the questionnaire. Also, it might be necessary to start with an active/inactive classification. On average it took the participants $3 \mathrm{~min}$ and $39 \mathrm{~s}$ to complete the continuous questionnaire. The start and end time of the completed questionnaires are recorded, so to ensure that the time the questionnaire was completed will not be included in the analysis. The selected timeframe will then be divided into windows of length $w$ from which different features in the time and spectral domain 
will be extracted. Similar to different timeframe lengths, different window lengths for feature extraction will need to be compared as well. The extracted features can then be used for correlation of wearable data with ESM data and eventually for prediction of ESM data. See Fig. 5 for an overview of the proposed data processing pipeline.

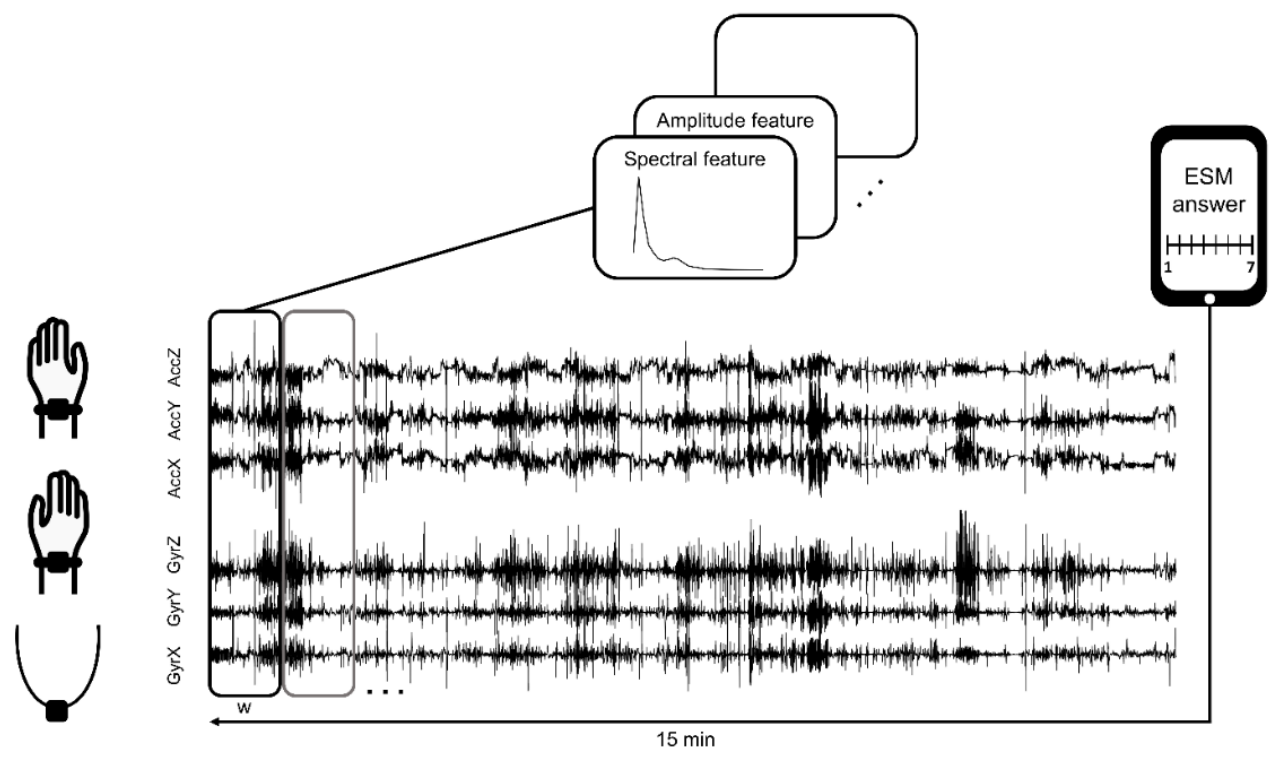

Figure 5. Proposed data processing steps. Fifteen minutes of sensor data prior to a completed questionnaire will be extracted. This timeframe will then be divided into windows of length $w$ from which different features in the time and spectral domain will be extracted.

One limitation of the used monitoring system is that due to minimizing the number of sensors not all PD symptoms can be measured. For example, tremor in the lower limbs will likely not be measured with this system. Future studies might consider the use of smart insoles as non-obstructive sensor to measure tremor and other symptoms related to the lower limbs.

This study combined wearable sensors and ESM in PD patients. We demonstrated that our newly developed PD monitoring system is feasible and that it can be used for the continuous measurement of PD symptoms during daily life for both monitoring and treatment purposes. Further, it breaks new ground since the system collects objective wearable sensor data as well as subjective ESM data which might be used as a parallel ground truth. Therefore, this monitoring system does not require additional clinical assessments. If correlating wearable and ESM data, and eventually predicting ESM scores based on wearable data succeeds, the monitoring system can be used to monitor the patient during daily life in periods of medication changes, or periods preceding an outpatient clinic visit. 


\section{Methods}

\section{Participants}

This study was approved by the medical ethical committee azM/UM and written informed consent was obtained from all participants. Twenty idiopathic PD patients participated in this study. Recruitment was done through their neurologist or neurosurgeon at the Maastricht University Medical Centre. Disease severity had to be rated as mild to severe (Hoehn and Yahr 1-4). Patients were included if they had an age between 18 and 80 years, if they were in possession of a smartphone (minimal iOS 8 or Android 4), if they could fluently speak and read Dutch, if they were available for two consecutive weeks of representative daily activities (meaning no holidays or planned hospital admission). After written informed consent, participants were tested for cognitive deficits and were excluded if they scored less than 24 points on the Montreal Cognitive Assessment. Participant characteristics are shown in Table 1. Hoehn and Yahr scores are shown in Fig. 6.

Table 1. Participant characteristics. Data are presented as mean (std)

$\begin{array}{ll}\text { Male/female, } \boldsymbol{n} & 16 / 4 \\ \text { Age, years } & 63.3(7.4) \\ \text { Montreal Cognitive Assessment score } & 27.6(1.5) \\ \text { Disease duration, years } & 8.1(5.8) \\ \text { Levodopa equivalent daily dose } & 770.4(393.9) \\ \text { Tremor/akinetic rigid, } \boldsymbol{n} & 9 / 11 \\ \text { ON/OFF fluctuations yes/no, } \boldsymbol{n} & 12 / 8 \\ \text { Deep brain stimulation yes/no, } \boldsymbol{n} & 6 / 14 \\ \text { Deep brain stimulation duration, years } & 3.3(1.5)\end{array}$

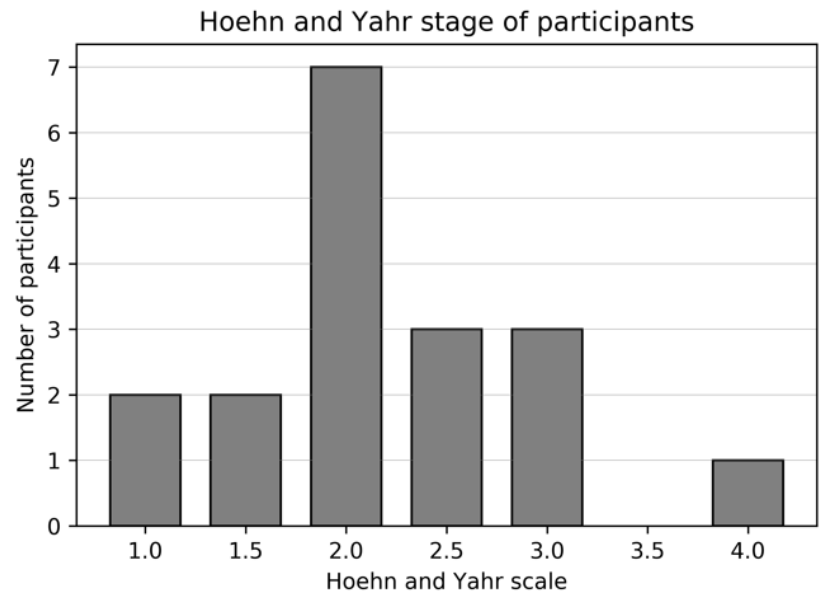

Figure 6. Disease severity of the participants indicated by Hoehn \& Yahr scores. 


\section{Wearable sensors}

We chose to use a new wearable, the MOX5, which was developed by the Instrument Development Engineering \& Evaluation department of the Maastricht University. This sensor received CE mark approval and is available for third parties via Maastricht Instruments (Maastricht, The Netherlands). We decided to develop a wearable instead of re-using existing ones because this study acquires access to the raw accelerometer and gyroscope data, and because the on-device data storage removed the need for batterydraining data transfers. Further, this sensor has the possibility to implement symptom algorithms and online data streaming in the future.

The developed sensor contained a 6 DOF sensor, consisting of an accelerometer and gyroscope. The accelerometer covered an amplitude range of $\pm 8 \mathrm{~g}$ and the gyroscope covered a range of \pm 2000 degrees/s. Data were collected with a sampling rate of $200 \mathrm{~Hz}$. During the measurement period, each participant had to wear three wearable sensors; one at each wrist and one at the chest. The wearable sensors were attached to the body via handmade accessories (Fig. 1a). The participants had to wear the wearable sensors during daytime (preferably between 08.00 and 22.00), and had to charge them at night. Wearable sensors were aligned to the ESM questionnaires using time stamps. The participants did not have to interact with the wearable, since the measurement started as soon as the charger was removed and data was later extracted by the research team.

\section{Experience sampling method}

An ESM app, the Psymate, was downloaded and installed on the smartphone of the participants. We developed a specific ESM questionnaire, based on literature discussing relevant symptoms and items for monitoring PD at home ${ }^{28,29}$ and based on patient and clinician interviews about relevant symptoms and items for PD monitoring at home. During the measurement period, participants were asked to complete a morning (five questions) and evening questionnaire (eight questions) which were identical on all days and which were available in the morning and evening (Supplementary Fig. 3). The morning and evening questionnaire were only available during specific timeframes and the participants were asked to complete the questionnaires when they woke up and when they were going to bed. In addition, they received a continuous questionnaire (26 questions) at seven semi randomized moments during the day (Supplementary Fig. 3). During each 2-hour block between 8.00 and $22.00 \mathrm{~h}$, one continuous questionnaire was sent. See Fig. 2 for an overview of one test day. The continuous questionnaires had to be opened within 15 minutes after the notification alarm and the participants were asked to complete as much questionnaires as possible without adapting their normal daily behaviour. Participants had to rate statement questions on a Likert scale ([1-7], Fig. 1b). Some questions were multiple-choice (Supplementary Fig. 3). 


\section{Combining ESM and wearable sensor data}

To investigate whether the ESM answers can be employed as a ground truth for the sensor data, we developed a prediction framework. In this framework, we used features calculated from the sensor data to predict OFF moments experienced by the patient. Based on previous literature ${ }^{30-32}$, the following features were extracted:

1. Logarithmic Signal Energy between 3.5 and $7.5 \mathrm{~Hz}$

2. Root Mean Square of the low-pass filtered $(3 \mathrm{~Hz})$ time series

3. Dominant Frequency and dominant energy ratio

4. Amplitude Range of the Raw Time series

5. Maximum Normalized Cross-correlation and corresponding temporal offset between all accelerometer and gyroscope channels

We evaluated our prediction framework in a 10 -fold cross-validation and employed a simple logistic regression classifier to output probabilities for OFF moments. For this pilot study, we only evaluated one patient reporting frequent ON/OFF transitions.

\section{Outcomes}

The feasibility of our PD monitoring system will be expressed in several outcomes. Participants completed an evaluation questionnaire including questions about the use of the wearable sensors and the ESM application and about the study in general. Results of this questionnaire are outcomes for the wearable sensors, ESM and the monitoring system in general.

For the wearable sensors, the outcomes are also the minutes of collected data. This will be divided into three categories: (1) Worn time within instructed timeframe; (2) Non-worn time within instructed timeframe; (3) Worn time outside the instructed timeframe. Since the wearable sensors recorded from the moment the charger was removed until the moment the charger was plugged in again, there will be time measured in which the participant did not wear the wearable. In addition, patients may have taken off the wearable sensors during daytime when they were for example taking a shower. In order to only select the data in which the wearable sensors were actually worn, the standard deviation of the acceleration data was calculated per minute block. This was calculated for data in the $x$ direction, which was parallel to the lower arm for the wrist sensors and which was parallel to the whole body for the chest sensor. A threshold value was empirically determined based on the standard deviation of non-worn wearable recordings. As a result, when the standard deviation per minute block was $<0.002 \mathrm{~g}$, and when the standard deviation of the preceding and following minute were $<0.002 \mathrm{~g}$ as well, the minute was not included in the minutes of collected data. Minutes of collected data were averaged over the three sensors, over days, and over participants. 
For the ESM, the outcomes are the percentages of completed questionnaires and the percentages of completed questionnaires for which sensor data from all three sensors was available as well. To evaluate the burden of each continuous questionnaire, we included a question on 'how disturbing' the corresponding questionnaire was. For the combination of ESM and sensor data, we evaluated the results of the cross-validation using the area under the curve of the Receiver-Operator-Characteristic. 


\section{Supplementary materials}

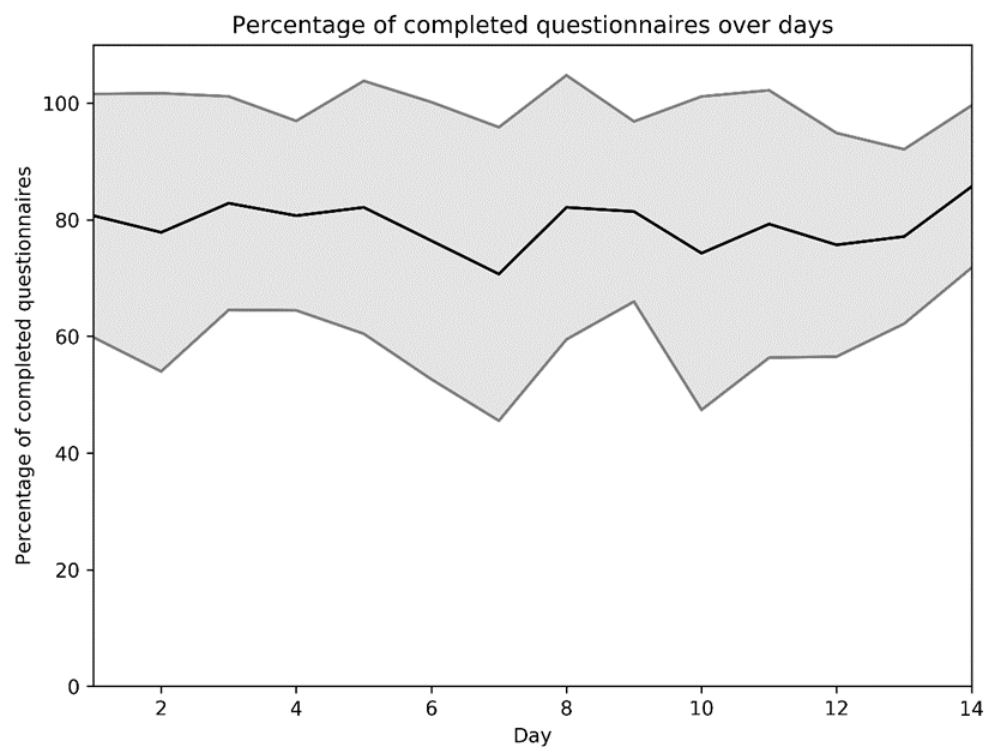

Supplementary Figure 1. Percentage of completed questionnaires over days. Light grey area is showing the standard deviation. A repeated measures ANOVA determined that completion rates did not differ significantly between days $(F(13,247)=0.97, p=0.49)$.

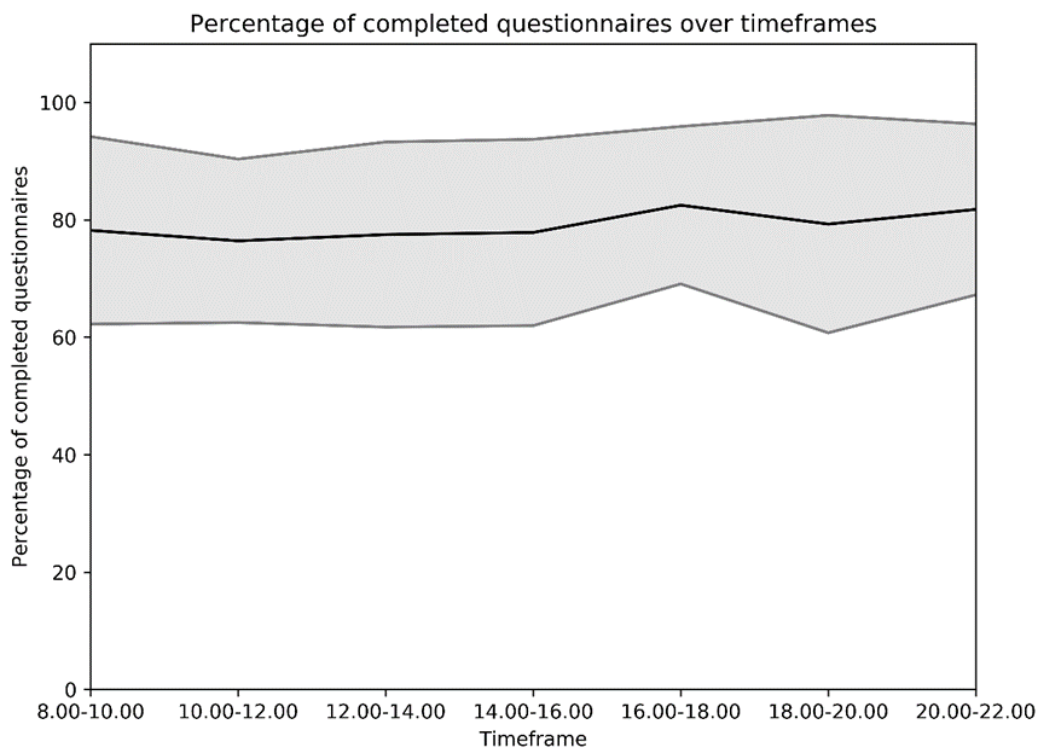

Supplementary Figure 2. Percentage of completed questionnaires over timeframes. Light grey area is showing the standard deviation. A repeated measures ANOVA determined that completion rates did not differ significantly between timeframes $(F(6,114)=1.20, p=0.31)$. 


\section{Continuous questionnaire:}

- I feel well

- I feel down

- I feel fearful

- I feel stressed

- I feel sleepy

- I am tired

- I am cheerful

- I am relaxed

- I can concentrate well

- I experience hallucinations

- I am at (home, work, travelling, at family/friends place, in public)

- I am (alone, with family, with my partner, with colleagues, with friends)

- I I am doing (work, resting, househole/odd jobs, sports, something else)

- I can do this without hinder

- I am comfortable walking/standing

- I can sit or stand still easily

- I can speak well

- I can walk well

- I experience tremor

- I am moving slow

- I experience stiffness

- I experience muscle tension

- I am moving uncontrollably

- I feel currently (OFF, OFF $\rightarrow$ ON, ON, ON $\rightarrow$ OFF)

- I took Parkinson medication since the last beep (yes, no, I don't recall)

- I found this beep disturbing

\section{Morning questionnaire:}

- I slept well

- I woke up often last night

- I feel rested

- It was physically difficult to get up

- It was mentally difficult to get up

\section{Evening questionnaire:}

- I had long OFF periods today

- I had many OFF periods today

- Walking went well today

- (Un)dressing went well today

- Eating and drinking went well today

- Personal care went well today

- Household activities went well today

- I was tired today

Supplementary Figure 3. Experience Sampling Method questionnaires. 


\section{References}

1. Goetz, C. G. et al. Movement Disorder Society-sponsored revision of the Unified Parkinson's Disease Rating Scale (MDS-UPDRS): scale presentation and clinimetric testing results. Mov Disord 23, 21292170 (2008).

2. Habets, J. G. V. et al. An update on adaptive deep brain stimulation in Parkinson's disease. Mov Disord 33, 1834-1843 (2018).

3. Rovini, E., Maremmani, C. \& Cavallo, F. How Wearable Sensors Can Support Parkinson's Disease Diagnosis and Treatment: A Systematic Review. Front Neurosci 11, 555 (2017).

4. Sánchez-Ferro, Á. et al. New methods for the assessment of Parkinson's disease (2005 to 2015): A systematic review. Mov Disord 31, 1283-1292 (2016).

5. Thorp, J. E., Adamczyk, P. G., Ploeg, H.-L. \& Pickett, K. A. Monitoring Motor Symptoms During Activities of Daily Living in Individuals With Parkinson's Disease. Front Neurol 9, 1036 (2018).

6. Basu, I. et al. Pathological tremor prediction using surface electromyogram and acceleration: potential use in 'ON-OFF' demand driven deep brain stimulator design. J Neural Eng 10, 036019 (2013).

7. Delrobaei, M. et al. Towards remote monitoring of Parkinson's disease tremor using wearable motion capture systems. J Neurol Sci 384, 38-45 (2018).

8. Khobragade, N., Graupe, D. \& Tuninetti, D. Towards fully automated closed-loop Deep Brain Stimulation in Parkinson's disease patients: A LAMSTAR-based tremor predictor. Annu Int Conf IEEE Eng Med Biol Soc 2015, 2616-2619 (2015).

9. Rodríguez-Martín, D. et al. Home detection of freezing of gait using support vector machines through a single waist-worn triaxial accelerometer. PLoS One 12, e0171764 (2017).

10. Griffiths, R. I. et al. Automated assessment of bradykinesia and dyskinesia in Parkinson's disease. J Parkinsons Dis 2, 47-55 (2012).

11. Delrobaei, M., Baktash, N., Gilmore, G., Mclsaac, K. \& Jog, M. Using Wearable Technology to Generate Objective Parkinson's Disease Dyskinesia Severity Score: Possibilities for Home Monitoring. IEEE Trans Neural Syst Rehabil Eng 25, 1853-1863 (2017).

12. Angeles, P., Tai, Y., Pavese, N., Wilson, S. \& Vaidyanathan, R. Automated assessment of symptom severity changes during deep brain stimulation (DBS) therapy for Parkinson's disease. IEEE Int Conf Rehabil Robot 2017, 1512-1517 (2017).

13. Bayés, À. et al. A 'HOLTER' for Parkinson's disease: Validation of the ability to detect on-off states using the REMPARK system. Gait Posture 59, 1-6 (2018).

14. Cancela, J. et al. Monitoring of motor and non-motor symptoms of Parkinson's disease through a mHealth platform. Annu Int Conf IEEE Eng Med Biol Soc 2016, 663-666 (2016).

15. Csikszentmihalyi, M. \& Larson, R. Validity and reliability of the Experience-Sampling Method. J Nerv Ment Dis 175, 526-536 (1987).

16. Myin-Germeys, I. et al. Experience sampling research in psychopathology: opening the black box of daily life. Psychol Med 39, 1533-1547 (2009).

17. Palmier-Claus, J. E. et al. Experience sampling research in individuals with mental illness: reflections and guidance. Acta Psychiatr Scand 123, 12-20 (2011).

18. van der Velden, R. M. J., Mulders, A. E. P., Drukker, M., Kuijf, M. L. \& Leentjens, A. F. G. Network analysis of symptoms in a Parkinson patient using experience sampling data: An $n=1$ study. Mov Disord 33, 1938-1944 (2018).

19. Vizcarra, J. A. et al. The Parkinson's disease e-diary: Developing a clinical and research tool for the digital age. Mov Disord 34, 676-681 (2019).

20. Bedard, C. et al. Understanding Environmental and Contextual Influences of Physical Activity During First-Year University: The Feasibility of Using Ecological Momentary Assessment in the MovingU Study. JMIR Public Health Surveill 3, e32 (2017).

21. Booij, S. H. et al. Cortisol and $\alpha$-Amylase Secretion Patterns between and within Depressed and NonDepressed Individuals. PLoS One 10, e0131002 (2015). 
22. Brannon, E. E., Cushing, C. C., Crick, C. J. \& Mitchell, T. B. The promise of wearable sensors and ecological momentary assessment measures for dynamical systems modeling in adolescents: a feasibility and acceptability study. Transl Behav Med 6, 558-565 (2016).

23. Knell, G. et al. Ecological Momentary Assessment of Physical Activity: Validation Study. J Med Internet Res 19, e253 (2017).

24. Liao, Y., Chou, C.-P., Huh, J., Leventhal, A. \& Dunton, G. Associations of Affective Responses During Free-Living Physical Activity and Future Physical Activity Levels: an Ecological Momentary Assessment Study. Int J Behav Med 24, 513-519 (2017).

25. Maher, J. P., Dzubur, E., Huh, J., Intille, S. \& Dunton, G. F. Within-Day Time-Varying Associations Between Behavioral Cognitions and Physical Activity in Adults. J Sport Exerc Psychol 38, 423-434 (2016).

26. Delespaul, P. a. E. G. Assessing schizophrenia in daily life : the experience sampling method. (1995).

27. Broen, M. P. G. et al. Unraveling the Relationship between Motor Symptoms, Affective States and Contextual Factors in Parkinson's Disease: A Feasibility Study of the Experience Sampling Method. PLoS One 11, e0151195 (2016).

28. Ferreira, J. J. et al. Clinical Parameters and Tools for Home-Based Assessment of Parkinson's Disease: Results from a Delphi study. J Parkinsons Dis 5, 281-290 (2015).

29. Serrano, J. A. et al. Participatory design in Parkinson's research with focus on the symptomatic domains to be measured. J Parkinsons Dis 5, 187-196 (2015).

30. Hoff, J. I., Wagemans, E. A. \& van Hilten, B. J. Ambulatory objective assessment of tremor in Parkinson's disease. Clin Neuropharmacol 24, 280-283 (2001).

31. Patel, S. et al. Monitoring motor fluctuations in patients with Parkinson's disease using wearable sensors. IEEE Trans Inf Technol Biomed 13, 864-873 (2009).

32. Salarian, A. et al. Quantification of tremor and bradykinesia in Parkinson's disease using a novel ambulatory monitoring system. IEEE Trans Biomed Eng 54, 313-322 (2007). 



\section{Chapter 4}

\section{Evaluation of Parkinson's disease at home: Predicting tremor from wearable sensors}

M. Heijmans*, J.G.V. Habets*, M.L. Kuijf, P.L. Kubben, C. Herff

* shared first authors

Published. Annual International Conference of the IEEE Engineering in Medicine and Biology Society 2019; 584-487 


\begin{abstract}
The continuous monitoring of Parkinsons's disease (PD) symptoms would allow to automatically adjust medication or deep brain stimulation parameters to a patient's momentary condition. Wearable sensors have been proposed to monitor PD symptoms and have been validated in a number of lab and hospital settings. However, taking these sensors into the daily life of patients introduces a number of difficulties, most notably the absence of an observable ground truth of what the user is currently doing. In this pilot study, we investigate PD symptoms by combining wearable sensors on both wrist and the chest with a questionnaire based evaluation of PD symptoms, in the form of experience sampling method. For a tremor dominant patient, we show that experienced tremor severity can be predicted from the sensor data with correlations of up to $r=0.43$. We evaluated different window lengths to calculate the features in and see better results for longer window lengths. Our results show that continuous monitoring of PD symptoms in daily life is feasible using wearable sensors.
\end{abstract}




\section{Introduction}

Parkinson's disease (PD) is a degenerative disorder in which a loss of dopaminergic neurons in the substantia nigra causes motor and non-motor symptoms. Cardinal motor symptoms are bradykinesia, rigidity, tremor and postural instability. The vast majority of PD patients is treated with dopaminergic medication, e.g. levodopa, to restore dopamine levels in the basal ganglia. A well-known challenge of this pharmacological treatment is the occurrence of intra-daily motor fluctuations and levodopa-induced dyskinesia after five to ten years ${ }^{1}$. These fluctuations are also known as ON- and OFF-fluctuations, where ON-state refers to periods during which motor symptoms are well treated, and OFF-state refers to periods during which motor symptoms are not treated sufficiently. Suffering from ON-OFF fluctuations can be an indication for deep brain stimulation (DBS), which then aims to increase the time a patient spends in ON-state. Therefore, monitoring these intra-daily fluctuations is of importance to control and adjust dopaminergic restoration therapy, and maybe even DBS in the future ${ }^{2}$.

Wearable sensors, in particular inertial measuring units consisting of accelerometers and/or gyroscopes, have potential to monitor PD symptoms and ON-OFF fluctuations in a continuous, non-obtrusive manner. Further validation of systems and preferably caremodels is needed before they are applicable for daily life monitoring ${ }^{3,4}$. Studies monitoring tremor during daily life activities are often limited because data were collected in simulated home settings in either a research lab or hospital ${ }^{5-9}$. Although the real-life representation of a simulated home setting stays debatable, videotape recordings of the assessments can be made and be used as parallel 'ground truth' data. Studies which are performed in the real home situation are however limited because of the lack of such parallel ground truth data ${ }^{3}$. This highlights one of the major challenges in PD monitoring; the availability of reliable ground truth data on the patients clinical state.

We introduce experience sampling method (ESM), also known as ecological momentary assessment, to provide additional subjective data on the patient's well-being to verify and evaluate our wearable sensor data ${ }^{10}$. The smartphone-based ESM method we used, presents digital questionnaires at semi-randomized moments throughout the day asking the patient about current motor state and symptoms. Additionally, there are accompanying questions on mood, affect and context, which can also fluctuate and cannot be measured directly from the wearable sensors. The development and validation of the ESM-method will be described elsewhere. In this case report, we describe a tremordominant PD patient who reported to suffer from ON-OFF fluctuations. We demonstrate how fluctuations in tremor severity, measured by ESM, can be predicted from wearable sensors during daily living. 


\section{Material and methods}

\section{Study Design}

During a period of two consecutive weeks, participants had to wear three wearable sensors (one at each wrist and one at the chest) and had to complete ESM questionnaires.

1) Wearables: This study used MOX5 wearables (Maastricht Instruments, Maastricht, The Netherlands), containing both an accelerometer and gyroscope. The accelerometer covered an amplitude range of $\pm 8 \mathrm{~g}$ and the gyroscope covered a range of $\pm 2000 \mathrm{deg} / \mathrm{s}$. Data were collected with a sampling rate of $200 \mathrm{~Hz}$. The wearables were attached to the body via handmade wristbands and a necklace (Fig. 1). Participants had to wear the wearables only during daytime, and had to charge them at night. For subsequent alignment to the ESM questionnaires, the data were time stamped on the devices. Data were saved on the device and were extracted by the research team after the measurement period.

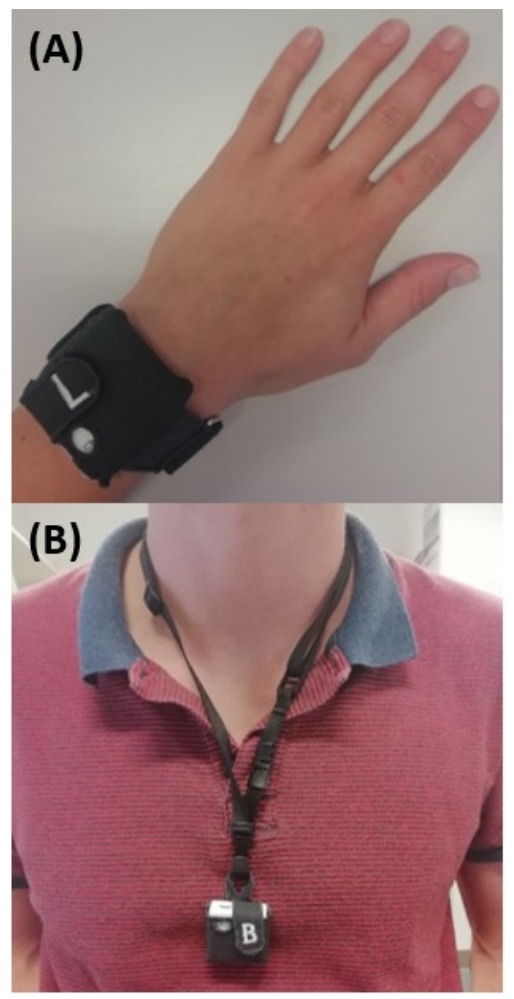

Figure 1. Wrist-worn (A) and chest (B) sensors including accelerometer and gyroscope as well as flash storage and batteries. Participants wear the sensors from waking up until they go to bed and charge them during the night. 
2) ESM: The ESM app Psymate was installed on the participants smartphone. We developed a specific PD questionnaire using previous work ${ }^{11,12}$ and by patient and clinician interviews about what is considered important when identifying inter-and intra-daily fluctuations in PD symptoms. During the measurement period, participants received repetitive questionnaires (containing 26 questions) at seven semi-randomized moments during the day. These questionnaires contained questions on mood, affect, context, motor state and PD symptoms at that specific moment (e.g. I experience tremor). Participants had to rate the questions on a 7-point Likert scale. The questionnaires stayed available for only 15 minutes, thereby excluding recall bias. The participants were asked to complete as much questionnaires as possible without adapting their normal daily behaviour.

\section{Participants}

This study was approved by the METC azM/UM and written informed consent was obtained from all participants. Recruitment was done by neurologists, neurosurgeons, and PD nurses at the Maastricht University Medical Centre. Inclusion criteria were: Diagnosed with idiopathic PD; in possession of a smartphone (iOS or android); mastering Dutch language; and being available for two consecutive weeks of representative daily activities. Patients were excluded if they scored less than 24 points on the Montreal Cognitive Assessment ${ }^{13}$. We included and finished data collection in 20 participants.

To be sure to have sufficient fluctuations in tremor severity for this pilot study, we investigate the case of a participant that reported to be tremor dominant and to suffer from severe ON-OFF fluctuations. This participant is a 65-year old male who has been diagnosed with idiopathic PD for 6 years. He scored 26 points on the Montreal Cognitive Assessment. Since one year this participant receives bilateral subthalamic DBS therapy. In addition to the DBS therapy, he takes dopaminergic medication five times a day (levodopa equivalent daily dose $=788$ ). The patient reported ON-OFF fluctuations which were medication related, resulting in periods of increased tremor ranging from approximately half an hour before standard medication intake moments until a quarter after medication intake.

\section{Data processing}

In order to align the continuous sensor data to the relevant subjective evaluations measured through ESM, we extracted the 15 minutes of sensor data prior to each completed questionnaire. We hypothesize that the previous 15 minutes best reflect the participants symptomatic experience. Each

of these 15 minute long blocks is then associated with the corresponding answers from the ESM questionnaire. To extract meaningful information from the sensor data, we extracted 
features both in the time and spectral domain ${ }^{14}$. We evaluate different window lengths for the feature extraction. Fig. 2 visualizes our feature extraction procedure.

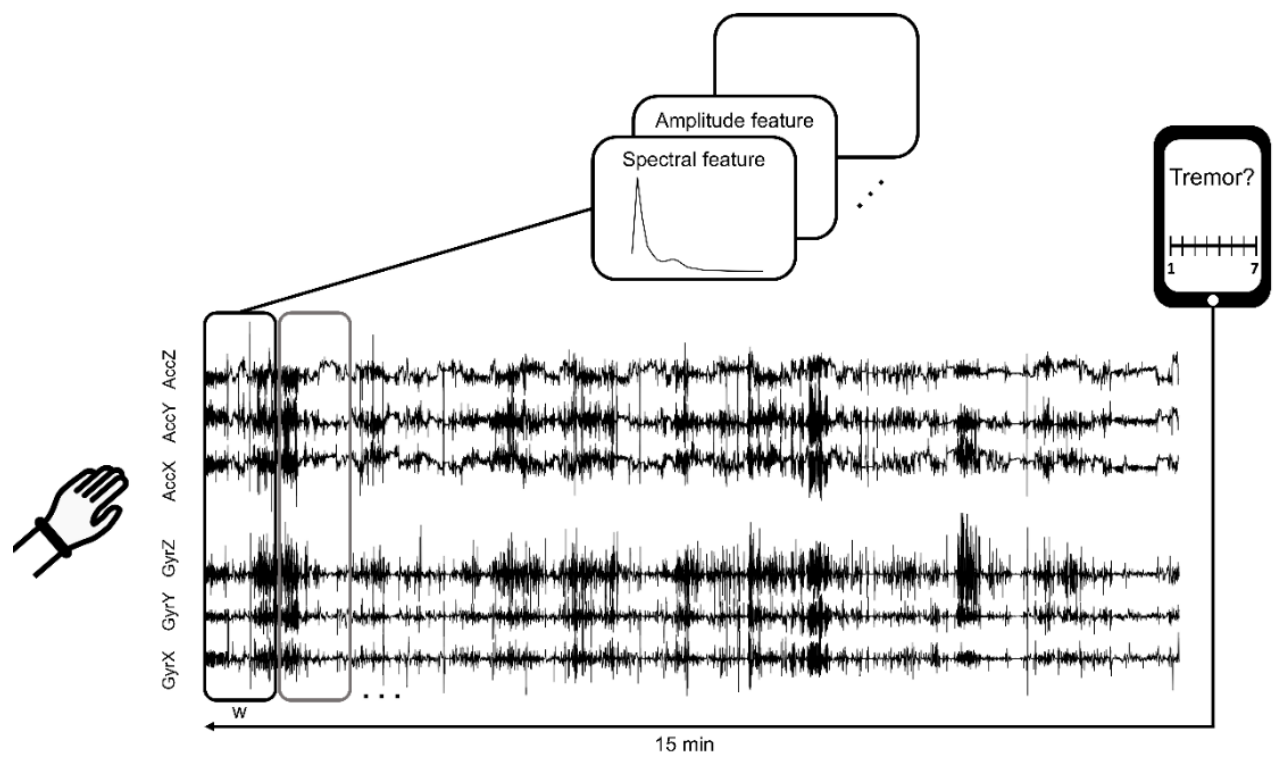

Figure 2. Feature extraction procedure. For each ESM questionnaire, we extract the proceeding 15 minutes of continuous sensor data. We divide these into windows of varying length $w$ and extract different features. The extracted feature vectors are then annotated with the answers from the corresponding questionnaire.

\section{Feature extraction}

Based on previous work $7,9,15$ in the detection and decoding of tremor and bradykinesia in lab or hospital settings, we extracted the following features:

1) Logarithmic signal energy in the $3.5-7.5 \mathrm{~Hz}$ frequency band. We extracted this information from both accelerometer and gyroscope data. (6 features)

2) Total signal energy in the form of the root mean squared signal. This feature was extracted from the low-pass filtered $(3 \mathrm{~Hz})$ accelerometer data. (3 features)

3) The dominant frequency in the low-pass filtered $(3 \mathrm{~Hz})$ accelerometer data. (3 features)

4) The dominant energy ratio, which we calculated by dividing the maximum energy by the total energy in the low-pass filtered $(3 \mathrm{~Hz})$ accelerometer data. (3 features)

5) The amplitude range, which we extracted from the lowpass filtered $(3 \mathrm{~Hz})$ time series of accelerometer data. (3 features) 
6) Maximum normalized cross-correlation and corresponding temporal offset among all pairs of low-pass filtered accelerometer time-series data. (2 features)

This results in a total of 20 features for each of the three wearables and thus a total of 60 features. To investigate the effect of window length and see which window length best represents the patient's answers, we extracted these features in non-overlapping windows of $30,60,120,180,300$ and 900 seconds. We assigned the same answers from the corresponding questionnaire to all windows extracted from one block of sensor data. Please note that this leads to different amounts of samples for the different window length conditions. While the 900 second long windows result in 62 samples (= number of filledout questionnaires), the 30 second long windows result in $62 * 30=1860$ samples. This also results in vastly different baseline levels, which we estimate using permutation levels (see Section Data Analysis).

\section{Data Analysis}

For this pilot study, we focused on both wrist worn sensors and excluded the chest sensor. This results in a total feature space of 40 . To test the prediction of tremor severity from the extracted features, we applied a 10 -fold cross-validation. In this approach, $90 \%$ of the data are used for training and the remaining $10 \%$ are used for testing, this is repeated until all samples have been used for testing exactly once. To predict the continuous severity assessments, we employed a simple linear regression. We calculated Pearson correlations between original and predicted tremor severity to evaluate the prediction.

We tested statistical significance through random permutation tests, in which we randomly shuffled the severity scores and calculated correlations between original and shuffled scores. This process was repeated 1000 times and the $95 \%$ highest correlations were used as a random baseline to signify the $\alpha=0: 05$-level.

\section{Results}

Correlation coefficients between original and predicted tremor scores are significantly above chance level for all window lengths except for the 120 second long windows (Fig. 3 (A)). This drop is further characterized by higher correlation coefficients for shorter (above 0.15 for both 30 and 60 seconds) and longer windows (180 seconds and above). Highest correlation scores were obtained for 15 minute long windows with $r=0: 43$. Fig. 3 (B) shows an example of predicted and actual tremor scores for 15 minute long windows. 
(A)

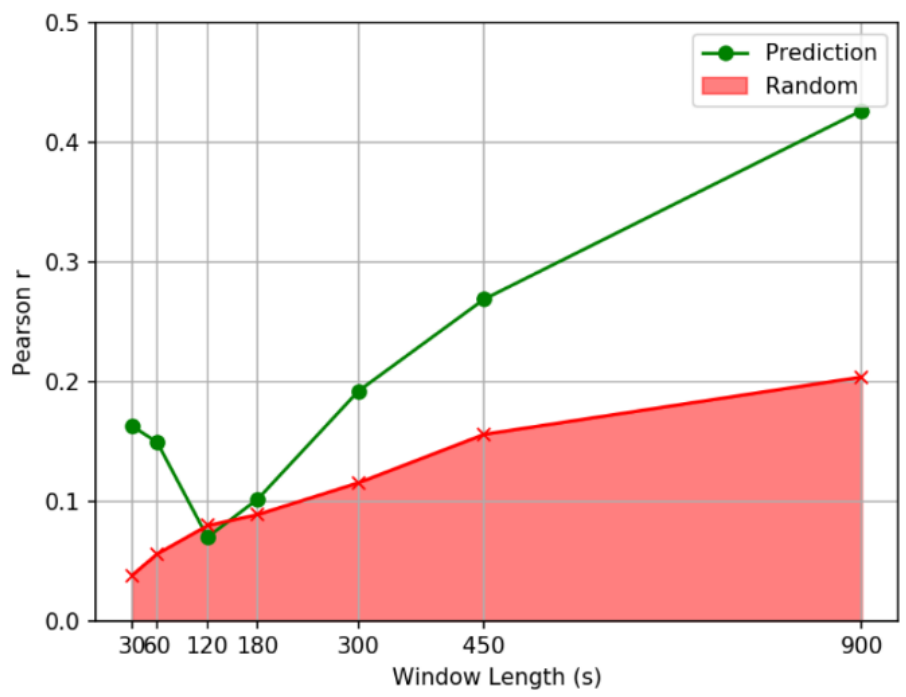

(B)

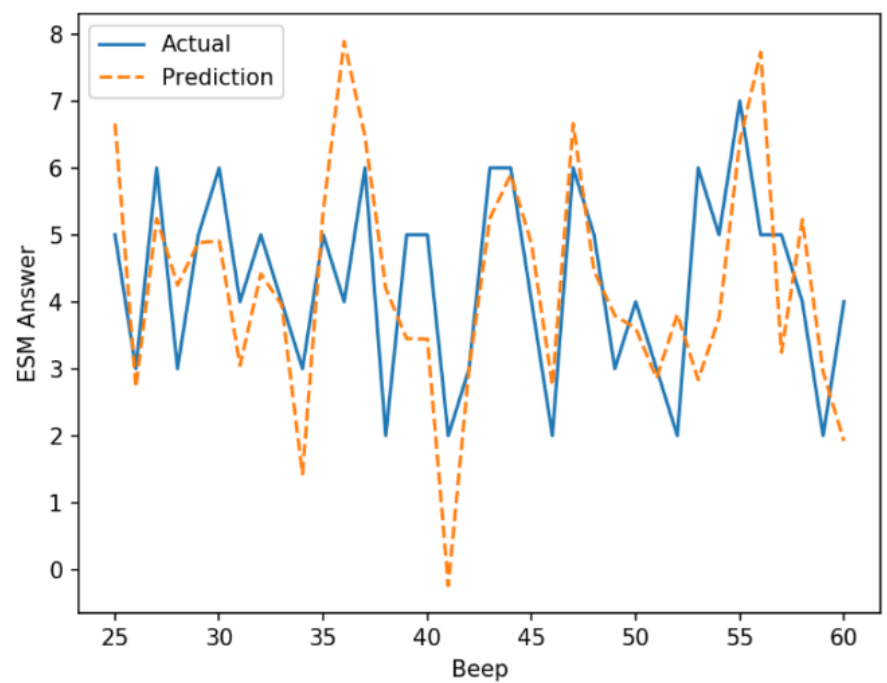

Fig. 3. Mean correlation results for tremor severity (A). Correlation coefficients for all but the 120 second window length are significantly above chance level (red shaded area, $\alpha=0: 05$ ). Example of actual (blue) and predicted (yellow) tremor scores for the full 15 minute window condition (B). 


\section{Discussion}

The evaluation of PD symptoms in continuous monitoring during daily life activities is very challenging, as no ground truth is available. Patients might sit still in front of the TV, be engaged in a conversation or take a walk in the park. These different activities will have tremendous effects on the measured sensor data and variations need to be taken into account before reliable prediction of symptoms can be achieved. Our preliminary results give compelling evidence that features that are known to be successful in the prediction of PD symptoms in lab settings ${ }^{15}$, can be used to predict tremor severity during the patients daily life. These results expand previous work which did make use of activity detection using videotapes, clinical assessments, and predefined motor tasks ${ }^{6-8,8,9}$. In addition, our results expand previous work which predicted clinical tremor scores from tremor data which was recorded while patients were comfortably seated in a chair ${ }^{16}$. Lastly, this study is to our knowledge the first one comparing the effect of using different window lengths for tremor feature extraction. Longer window lengths resulted in better correlation results of the predicted ESM scores. It is important to note that the chance level results are also higher for longer windows, as the number of samples decreases and high correlations are more likely in smaller sample sizes.

Clearly, our results need to be extended to more patients with different amounts of ONOFF fluctuations and to other PD motor symptoms, such as bradykinesia, rigidity, and dyskinesia.

\section{Conclusion}

In this case report, investigating fluctuating PD tremor in a patient's daily life, we show that subjective tremor scores can be predicted with good correlations from wrist-worn sensors using very simple regression models. We employ standard features from the literature and investigate the influence of different window lengths on the prediction quality. For analyses of this individual patient, longer window lengths seem to result in better prediction quality. This confirms the possibility of, and increases the knowledge on monitoring PD symptoms in a daily life situation. 


\section{References}

1. Jankovic, J. Motor fluctuations and dyskinesias in Parkinson's disease: clinical manifestations. Mov Disord 20 Suppl 11, S11-16 (2005).

2. Habets, J. G. V. et al. An update on adaptive deep brain stimulation in Parkinson's disease. Mov Disord 33, 1834-1843 (2018).

3. Rodríguez-Molinero, A. et al. A Kinematic Sensor and Algorithm to Detect Motor Fluctuations in Parkinson Disease: Validation Study Under Real Conditions of Use. JMIR Rehabil Assist Technol 5, (2018).

4. Thorp, J. E., Adamczyk, P. G., Ploeg, H.-L. \& Pickett, K. A. Monitoring Motor Symptoms During Activities of Daily Living in Individuals With Parkinson's Disease. Front Neurol 9, 1036 (2018).

5. Cole, B. T., Roy, S. H., De Luca, C. J. \& Nawab, S. Dynamic neural network detection of tremor and dyskinesia from wearable sensor data. Annu Int Conf IEEE Eng Med Biol Soc 2010, 6062-6065 (2010).

6. Cole, B. T., Roy, S. H., De Luca, C. J. \& Nawab, S. H. Dynamical learning and tracking of tremor and dyskinesia from wearable sensors. IEEE Trans Neural Syst Rehabil Eng 22, 982-991 (2014).

7. Hoff, J. I., Wagemans, E. A. \& van Hilten, B. J. Ambulatory objective assessment of tremor in Parkinson's disease. Clin Neuropharmacol 24, 280-283 (2001).

8. Roy, S. H., Cole, B. T., Gilmore, L. D., De Luca, C. J. \& Nawab, S. H. Resolving signal complexities for ambulatory monitoring of motor function in Parkinson's disease. Annu Int Conf IEEE Eng Med Biol Soc 2011, 4832-4835 (2011).

9. Salarian, A. et al. Quantification of tremor and bradykinesia in Parkinson's disease using a novel ambulatory monitoring system. IEEE Trans Biomed Eng 54, 313-322 (2007).

10. Brannon, E. E., Cushing, C. C., Crick, C. J. \& Mitchell, T. B. The promise of wearable sensors and ecological momentary assessment measures for dynamical systems modeling in adolescents: a feasibility and acceptability study. Transl Behav Med 6, 558-565 (2016).

11. Ferreira, J. J. et al. Clinical Parameters and Tools for Home-Based Assessment of Parkinson's Disease: Results from a Delphi study. J Parkinsons Dis 5, 281-290 (2015).

12. Serrano, J. A. et al. Participatory design in Parkinson's research with focus on the symptomatic domains to be measured. J Parkinsons Dis 5, 187-196 (2015).

13. Nasreddine, Z. S. et al. The Montreal Cognitive Assessment, MoCA: a brief screening tool for mild cognitive impairment. J Am Geriatr Soc 53, 695-699 (2005).

14. Herff, C. \& Krusienski, D. J. Extracting Features from Time Series. in Fundamentals of Clinical Data Science (eds. Kubben, P., Dumontier, M. \& Dekker, A.) (Springer, 2019).

15. Patel, S. et al. Monitoring motor fluctuations in patients with Parkinson's disease using wearable sensors. IEEE Trans Inf Technol Biomed 13, 864-873 (2009).

16. Jeon, H. et al. Automatic Classification of Tremor Severity in Parkinson's Disease Using a Wearable Device. Sensors (Basel) 17, (2017). 


\section{Part 2: Using MRI to track and treat Parkinson's disease}





\section{Chapter 5}

\section{The TRACK-PD study: protocol of a}

longitudinal ultra-high field imaging study in Parkinson's disease

A.F. Wolters, M. Heijmans, S. Michielse, A.F.G Leentjens, A.A. Postma, J.F.A. Jansen, D. Ivanov, A.A. Duits, Y. Temel, M.L. Kuijf

Published. BMC Neurology 2020; 20:292 


\section{Abstract \\ Background}

The diagnosis of Parkinson's Disease (PD) remains a challenge and is currently based on the assessment of clinical symptoms. PD is also a heterogeneous disease with great variability in symptoms, disease course, and response to therapy. There is a general need for a better understanding of this heterogeneity and the interlinked long-term changes in brain function and structure in PD. Over the past years there is increasing interest in the value of new paradigms in Magnetic Resonance Imaging (MRI) and the potential of ultra-high field strength imaging in the diagnostic work-up of PD. With this multimodal 7 T MRI study, our objectives are: 1) To identify distinctive MRI characteristics in PD patients and to create a diagnostic tool based on these differences. 2) To correlate MRI characteristics to clinical phenotype, genetics and progression of symptoms. 3) To detect future imaging biomarkers for disease progression that could be valuable for the evaluation of new therapies.

\section{Methods}

The TRACK-PD study is a longitudinal observational study in a cohort of 130 recently diagnosed ( $\leq 3$ years after diagnosis) PD patients and 60 age-matched healthy controls (HC). A 7 T MRI of the brain will be performed at baseline and repeated after 2 and 4 years. Complete assessment of motor, cognitive, neuropsychiatric and autonomic symptoms will be performed at baseline and follow-up visits with wearable sensors, validated questionnaires and rating scales. At baseline a blood DNA sample will also be collected.

\section{Discussion}

This is the first longitudinal, observational, $7 \mathrm{~T} \mathrm{MRI} \mathrm{study} \mathrm{in} \mathrm{PD} \mathrm{patients.} \mathrm{With} \mathrm{this} \mathrm{study,} \mathrm{an}$ important contribution can be made to the improvement of the current diagnostic process in PD. Moreover, this study will be able to provide valuable information related to the different clinical phenotypes of PD and their correlating MRI characteristics. The long-term aim of this study is to better understand PD and develop new biomarkers for disease progression which may help new therapy development. Eventually, this may lead to predictive models for individual PD patients and towards personalized medicine in the future. 


\section{Background}

Parkinson's disease (PD) is the second most common neurodegenerative disorder after Alzheimer's disease and is characterized by motor symptoms such as bradykinesia, rigidity and tremor ${ }^{1-3}$. Patients with PD also experience a broad spectrum of non-motor symptoms such as anxiety, depression, pain and autonomic dysfunction, making the disease a typical example of a neuropsychiatric disorder. The diagnosis of PD is currently based on the assessment of clinical symptoms and their course over time. However, the early diagnosis of PD can be challenging since its presentation is heterogeneous and mild symptoms are often not immediately recognised. Clinico-pathological research shows that the error rate for a clinical diagnosis of PD can be as high as $24 \%$, even in specialized centres ${ }^{4}$.

Recently, clinical criteria have been revised for the diagnosis of $\mathrm{PD}^{3}$ and several subtypes of PD are being recognized ${ }^{5,6}$. Currently a Movement Disorder Society (MDS) task force is mandated to review the evidence for these subtypes and propose a subtype classification system. The different subtypes of PD might be influenced by a combination of environmental and genetic factors ${ }^{7}$. However, the underlying aetiology of the clinical heterogeneity in PD is not well understood ${ }^{8}$. This is why the National Institutes of Health (NIH) pointed out that obtaining more insight in this heterogenous nature and defining the different PD subtypes, is one of the top three research priorities in PD ${ }^{9}$.

In clinical practice, Magnetic Resonance Imaging (MRI) is currently used as a method to exclude other potential causes of parkinsonian symptoms. To date, it is impossible to diagnose a patient with PD based on MRI characteristics. However, over the past years it has become well-established that MRI may serve as a valuable method in the diagnostic work-up of PD ${ }^{10}$. Early indicators, such as signal loss of the nigrosome-1 area on ironsensitive MR Images and reduced volume and signal intensity of the substantia nigra on neuromelanin-sensitive images, have been described in PD ${ }^{11-14}$. Additionally, functional MRI (fMRI) techniques can display changes related to specific symptoms in PD ${ }^{15,16}$. With the emergence of ultra-high-field scanners (7 T and above) submillimetre anatomical information can be obtained. Compared with $3 \mathrm{~T}$ MRI, ultra-high-field MRI at 7 T provides an increased spatial resolution and a higher signal-to-noise ratio enabling a potential higher degree of diagnostic detail ${ }^{10}$.

The primary objective of this study is to identify early and subtle MRI changes in PD patients which distinguish them from the healthy population and to create a reliable tool for the early diagnosis of PD based on these differences. Secondary objectives are to detect whether different clinical phenotypes of PD patients also show different imaging characteristics and to design a prognostic tool for individual PD patients by correlating specific MRI characteristics to clinical phenotype, genetic characteristics and progression 
of symptoms. Moreover, this large longitudinal ultra-high field imaging study may eventually also find new imaging biomarkers for disease progression that could be valuable for the development and evaluation of new therapies in PD. In addition, the database will serve as a biobank for further related research.

\section{Methods}

This is a longitudinal observational study in PD patients and healthy controls (HC). The TRACK-PD study (www.trackpd.nl) will assess the structural and functional characteristics of PD patients on ultra-high field 7 T MRI. The study has been registered at the Dutch Trial Register (www.trialregister.nl) with identification number NL7558.

\section{Participants}

In this study, 130 participants with PD will be recruited from the PD population visiting the movement disorder clinic of the Department of Neurology of the Maastricht University Medical Centre and other collaborating hospitals. In addition, we will use other media, such as websites, social media and patient meetings to recruit patients. $60 \mathrm{HC}$ participants will be recruited through advertisements in the hospital and university.

\section{Inclusion and exclusion criteria}

Participants are eligible for participation in this study if they meet the following criteria: 1) All patients have to be diagnosed with PD by a neurologist, within the last 3 years before inclusion. 2) A score of $\geq 24$ on the Montreal Cognitive Assessment (MoCA) at baseline. 3) Able to read and understand Dutch. 4) 18 years of age or older. 5) Providing written informed consent.

Participants with advanced cognitive impairment, defined as a score of $<24$ on the Montreal Cognitive Assessment (MoCA), or a diagnosis of dementia according to the fifth edition of the Diagnostic and Statistical Manual of Mental Disorders (DSM 5, ${ }^{17}$ ) criteria at baseline, will be excluded from participation. The presence of a clear diagnosis of neurodegenerative diseases other than PD is also an exclusion criterion. Lastly, potential participants cannot take part if there are any contra-indications for a $7 \mathrm{~T} \mathrm{MRI} \mathrm{scan,} \mathrm{such} \mathrm{as}$ claustrophobia, permanent makeup or the presence of incompatible metallic devices in their body. These exclusion criteria are also in place for the HC group.

\section{Study procedure}

This is a longitudinal observational study in which all participants will be tracked for 4 years. All data will be collected in one academic hospital in the Netherlands (Maastricht University Medical Centre). A 7 T brain MRI will be conducted at baseline, after 2 years and after 4 years. Basic clinical and demographic information, such as age, sex, handedness, disease 
duration, and the total levodopa equivalent daily dose (LEDD, ${ }^{18}$ ) will also be collected. Motor, cognitive, autonomic and neuropsychiatric symptoms will all be assessed by validated questionnaires and rating scales as summarized in Table 1. The assessments have been aligned with other national and international PD cohort studies, to permit future cross validation studies ${ }^{19,20}$. Wearable sensors measuring movement will be applied at each wrist and at the chest during the assessment days. In addition to the tests described above, a blood sample from all participants will be collected at baseline, which will be used for genetic and epigenetic testing on genes related to PD. Providing a blood sample is not mandatory for participation.

\section{Clinical assessments}

\section{Motor assessment}

Motor functions will be assessed with the unified Parkinson's Disease rating scale (MDSUPDRS) and the Hoehn and Yahr scale (H\&Y). The H\&Y is the most commonly used scale to estimate the global disease stage of PD patients ${ }^{21}$. The MDS-UPDRS consists of four parts, assessing both non-motor and motor disabilities in PD ${ }^{22}$. All four parts will be assessed by trained and certified investigators. At baseline, the UPDRS part III will also be used to check whether patients meet the MDS clinical diagnostic criteria for parkinsonism ${ }^{3}$. The motor evaluation is performed in medication ' $\mathrm{ON}^{\prime}$ ' state.

Furthermore, wearable sensor data will be collected on all testing days. The wearables contain both an accelerometer and a gyroscope and are described in more detail elsewhere ${ }^{23}$. During each testing day, the wearables will be put on after signing the informed consent form and will be removed while preparing the participant for the $7 \mathrm{~T} \mathrm{MRI}$. The wearables will be applied to the wrists by using comfortable straps. A hanger will be used for the chest wearable.

\section{Autonomic assessment}

The Scales for Outcomes in Parkinson's Disease - Autonomic dysfunction (SCOPA-AUT) questionnaire will be applied to assess autonomic functions in all participants. This questionnaire consists of 25 items assessing several different autonomic symptoms in patients with PD. It is a reliable and valid questionnaire for the evaluation of autonomic dysfunction in PD 24 .

\section{Neuropsychological assessment}

At baseline, global cognitive function is assessed with the MoCA, which is a 30 point screening tool, including several cognitive domains ${ }^{25}$. If, at baseline the MoCA score is $\geq 24$, the participant can continue the study protocol and a full neuropsychological assessment will be performed. 
Table 1. Overview of included study measures and scales in the TRACK-PD study

\begin{tabular}{|c|c|c|c|c|c|}
\hline Method & Outcome & Scales & $\begin{array}{l}\text { Visit } 1 \\
\text { (Baseli } \\
\text { ne) }\end{array}$ & $\begin{array}{l}\text { Visit } 2 \\
(2 \\
\text { Years) } \\
\times\end{array}$ & $\begin{array}{l}\text { Visit } 3 \\
\text { (4 } \\
\text { Years) } \\
\times\end{array}$ \\
\hline \multirow[t]{15}{*}{$\begin{array}{l}\text { Assessed by } \\
\text { trial assessor }\end{array}$} & $\begin{array}{l}\text { Motor functioning } \\
\text { in 'ON' state }\end{array}$ & $\begin{array}{l}\text { MDS-UPDRS III (including } \\
\text { H\&Y stage) }\end{array}$ & $X^{*}$ & $X^{*}$ & $X^{*}$ \\
\hline & & MDS-UPDRS IV & $\mathrm{X}^{*}$ & $X^{*}$ & $X^{*}$ \\
\hline & $\begin{array}{l}\text { Neuropsychologic } \\
\text { al symptoms }\end{array}$ & MoCA & $x$ & $\mathrm{X}$ & $x$ \\
\hline & & $\begin{array}{l}\text { Phonemic and semantic } \\
\text { fluency }\end{array}$ & $x$ & $\mathrm{X}$ & $\mathrm{X}$ \\
\hline & & 15 Words Test & $x$ & $x$ & $x$ \\
\hline & & $\begin{array}{l}\text { Benton Judgment of Line } \\
\text { Orientation }\end{array}$ & $x$ & $x$ & $x$ \\
\hline & & Letter Number Sequencing & $x$ & $\mathrm{X}$ & $x$ \\
\hline & & $\begin{array}{l}\text { Symbol Digit Modalities } \\
\text { Test }\end{array}$ & $X$ & $x$ & $x$ \\
\hline & & MDS-UPDRS I & $X^{*}$ & $X^{*}$ & $X^{*}$ \\
\hline & $\begin{array}{l}\text { Demographics } \\
\text { and lifestyle }\end{array}$ & Medical history & $X$ & $X$ & $X$ \\
\hline & & Medication & $x$ & $x$ & $x$ \\
\hline & Biospecimen & EDTA Plasma (DNA) & $X$ & & \\
\hline & & Pax Gene (RNA) & $x$ & & \\
\hline & $\begin{array}{l}\text { Brain structure } \\
\text { and function }\end{array}$ & $\begin{array}{l}\text { Resting-state functional } \\
\text { MRI }\end{array}$ & $x$ & $x$ & $x$ \\
\hline & & $\begin{array}{l}\text { Structural MRI (T1, T2*, } \\
\text { neuromelanin, DWI) }\end{array}$ & $x$ & $X$ & $x$ \\
\hline $\begin{array}{l}\text { Wearable } \\
\text { sensors }\end{array}$ & Motor parameters & $\begin{array}{l}\text { IMU including 3-axis } \\
\text { accelerometer and 3-axis } \\
\text { gyroscope }\end{array}$ & $x$ & $\mathrm{X}$ & $x$ \\
\hline \multirow{7}{*}{$\begin{array}{l}\text { Self-reported } \\
\text { patient } \\
\text { questionnaires }\end{array}$} & $\begin{array}{l}\text { Neuropsychiatric } \\
\text { symptoms }\end{array}$ & $\mathrm{BDI}$ & $x$ & $x$ & $x$ \\
\hline & & QUIP-RS & $X$ & $\mathrm{X}$ & $x$ \\
\hline & & PAS & $x$ & $x$ & $x$ \\
\hline & Quality of life & PDQ-8 & $\mathrm{X}^{*}$ & $\mathrm{X}^{*}$ & $X^{*}$ \\
\hline & $\begin{array}{l}\text { Autonomic } \\
\text { symptoms }\end{array}$ & SCOPA-AUT & $x$ & $x$ & $x$ \\
\hline & Sleep disorders & RBDQ & $X$ & $X$ & $x$ \\
\hline & Various & MDS-UPDRS II & $X^{*}$ & $\mathrm{X}^{*}$ & $X^{*}$ \\
\hline
\end{tabular}

BDI Beck Depression Inventory, HC healthy controls, H\&Y Hoehn and Yahr scale, IMU Inertial Measurement Unit, MDS-UPDRS Movement Disorders Society Unified Parkinson Disease Rating Scale, MoCA Montreal Cognitive Assessment, NPA Neuropsychological assessment, PAS Parkinson Anxiety Scale, PD Parkinson's disease patients, PDQ-8 Parkinson's Disease Questionnaire, QUIP-RS Questionnaire for Impulsive-Compulsive Disorders in Parkinson's disease, RBDSQ REM-sleep behaviour disorder screening questionnaire, SCOPA-AUT Scales for Outcomes in Parkinson's Disease - Autonomic dysfunction questionnaire. ${ }^{\times} \pm 60$ days; * Only for PD patients 
If the MoCA score drops below 24 during follow-up, participants will remain in the study. Neuropsychological assessment consists of the following standard tests: 1 . The 'Phonemic and Semantic Fluency Test' for executive function ${ }^{26} ; 2$. The 'Auditory Verbal Learning Test' for memory ${ }^{27} ; 3$. The 'Benton Judgment of Line Orientation' for visuospatial function ${ }^{28} ; 4$. The 'Symbol Digit Modalities Test' for mental speed and attention ${ }^{29}$; and 5. The 'Letter Number Sequencing Test' for working memory ${ }^{30}$.

\section{Neuropsychiatric assessment}

In addition, several questionnaires will assess psychiatric symptoms. The 'Beck Depression Inventory' (BDI), a 21-item questionnaire, is used to assess depression ${ }^{31}$. The Parkinson Anxiety Scale (PAS) ${ }^{32}$ is used to assess anxiety. The 'Questionnaire for ImpulsiveCompulsive Disorders' in Parkinson's disease (QUIP-RS) will assess impulsive and compulsive behaviours ${ }^{33}$. The Parkinson's Disease Questionnaire (PDQ-8) assesses quality of life of PD patients ${ }^{34}$. For each questionnaire the total sum score will be calculated.

\section{REM-sleep behaviour disorders}

Symptoms related to REM-sleep behaviour disorders will be assessed by using the 'REMsleep behaviour disorder screening questionnaire' (RBDSQ). This questionnaire is a 13-item screening tool for the detection of REM sleep behaviour disorders ${ }^{35}$. A total score will be calculated.

\section{Genetic testing}

Several contributing factors for PD risk, onset, and progression of symptoms have been associated with genetic variants. Especially in early-onset PD, strong penetrant mutations have been found. Possible new MRI correlates with known genetic variants could be valuable in further understanding the genetic basis and progression of PD. However, more than 20 mutations in genes have been associated with PD onset and more than 90 independent risk-associated variants in genome-wide association studies have been reported ${ }^{36}$. Blood samples of the participants will be tested on common genetic variants and stored in a database for further epigenetic testing, including the examination of DNA methylation patterns ${ }^{37}$. Drawing of the blood samples will be performed only by researchers or research nurses who are certified to do this. From every participant two ethylenediamine tetra-acetic acid buffered (EDTA) tubes with a size of $4.5 \mathrm{ml}$ each and two PAXgene ${ }^{\circledR}$ tubes with a size of $2.5 \mathrm{ml}$ each will be collected. These blood samples will be transferred to the BioBank of Maastricht University Medical Centre, were they will be stored at $-80^{\circ} \mathrm{C}$. 


\section{MRI acquisition}

Participants will be scanned on a 7 T MRI scanner (Magnetom, Siemens, Erlangen, Germany) equipped with a Nova Medical 32-channel head coil. Dielectric pads will be applied to enhance the signal in the temporal brain regions ${ }^{38}$. In PD patients, the MRI will be carried out while they are in the $\mathrm{ON}$-medication state in order to reduce the possibility of motion artefacts due to tremor and to reflect clinical practice. During the scan session participants will also watch a movie to reduce movements. Cardiac and respiratory physiological signals will be measured synchronized with the scan start. A localizer sequence will be acquired for optimal planning. B0 and B1 mapping and shimming will be performed to correct for field inhomogeneities. The scan protocol consists of 1) a wholebrain MP2RAGE (Magnetization Prepared 2 Rapid Acquisition Gradient Echoes) scan with an acquisition time of 10:57 min, resulting in a T1-weighted image and a quantitative T1 map. This MP2RAGE is combined with a SA2RAGE (Saturation Prepared with 2 Rapid Gradient Echoes) scan of 2:40 min, to eliminate any B1-related biases from the results, which can reduce the accuracy of the measurements ${ }^{39}$, 2) A multi-echo GRE, $4.8 \mathrm{~cm}$ coverage, acquisition time 7:42 min, used to provide susceptibility $\left(\mathrm{T} 2{ }^{*}\right)$-weighted images and $\mathrm{T} 2^{*}$ maps. In addition, quantitative susceptibility maps can be reconstructed from the same acquisition. This scan is sensitive for measuring variations in iron concentration (Field of View (FoV) is shown in Fig. 1), 3) A magnetization transfer-weighted TFL (MTW TFL), 3 $\mathrm{cm}$ coverage, acquisition time 4:38 $\mathrm{min}$, which can be used to visualise myelination or nuclei containing neuromelanin such as the substantia nigra and locus coeruleus ${ }^{40}$ (FoV is shown in Fig. 2), 4) A whole-brain diffusion weighted scan along 66 random directions with an average b-value of $2000 \mathrm{~s} / \mathrm{mm} 2$ mixed with six B0-volumes and one additional BOvolume and five diffusion weighted volume recorded with opposite phase-encoding direction, acquisition time 9:48 $\mathrm{min}, 5)$ A whole brain resting-state fMRI scan with 280 volumes and an additional five volumes recorded with reverse phase-encoding direction. Acquisition time for the resting-state $\mathrm{fMRI}$ is $11: 12 \mathrm{~min}$. During the resting-state $\mathrm{fMRI}$ scan participants are instructed to focus on a crosshair projected on a screen while letting their mind wander and not to think about anything in particular. In total the scan protocol takes a little less than 60 min and details can be found in Table 2.

\section{Sample size calculation}

Previous longitudinal MRI studies in PD have included a variety of sample sizes, but most studies contain about 15 to 25 patients and HC ${ }^{41-43}$. One larger longitudinal 3 T MRI study, the ICICLE-PD, recruited 105 PD patients and 37 matched $\mathrm{HC}$ and followed their participants for 18 months ${ }^{44}$. So far, no longitudinal $7 \mathrm{~T}$ studies nor $7 \mathrm{~T}$ MRI studies combining multiple MRI sequences in PD patients have been published. However, several cross-sectional $7 \mathrm{~T}$ MRI studies have been performed, all consisting of relatively small patient groups varying from 13 to 36 PD patients ${ }^{45-49}$. 


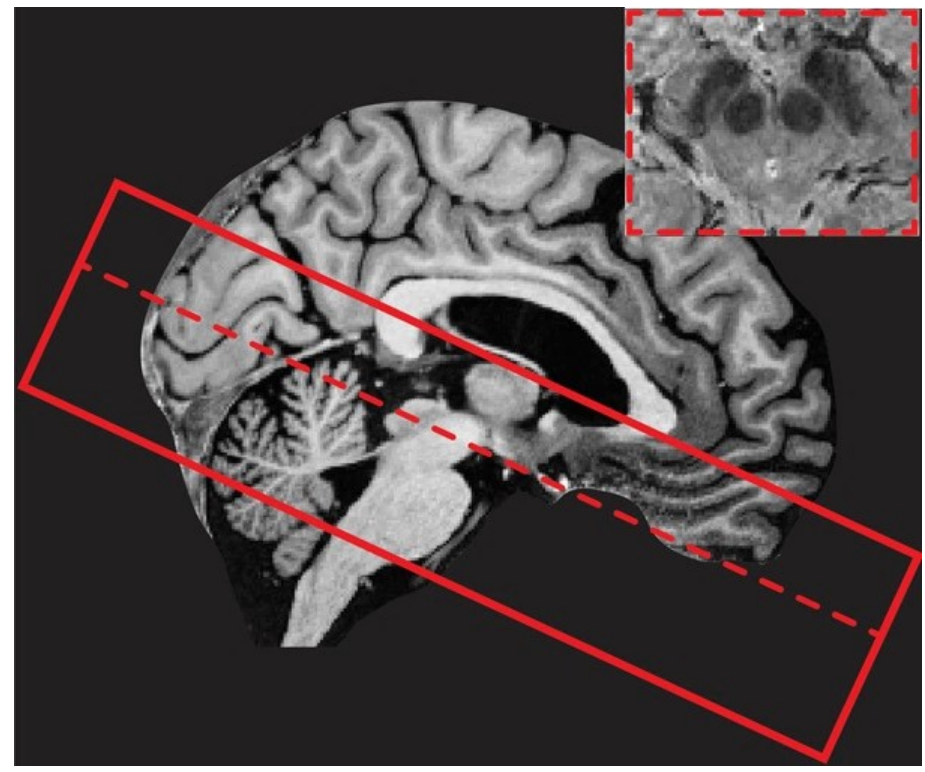

Figure 1. Visualisation of the multi-echo GRE field-of-view (FoV) with $4.8 \mathrm{~cm}$ coverage. The FoV is placed perpendicular to the brainstem. The inferior border of the FoV is positioned at the bottom of the 4th ventricle. Furthermore, an example of the multi-echo GRE transverse brain stem slice at the substantia nigra is displayed in the right corner.

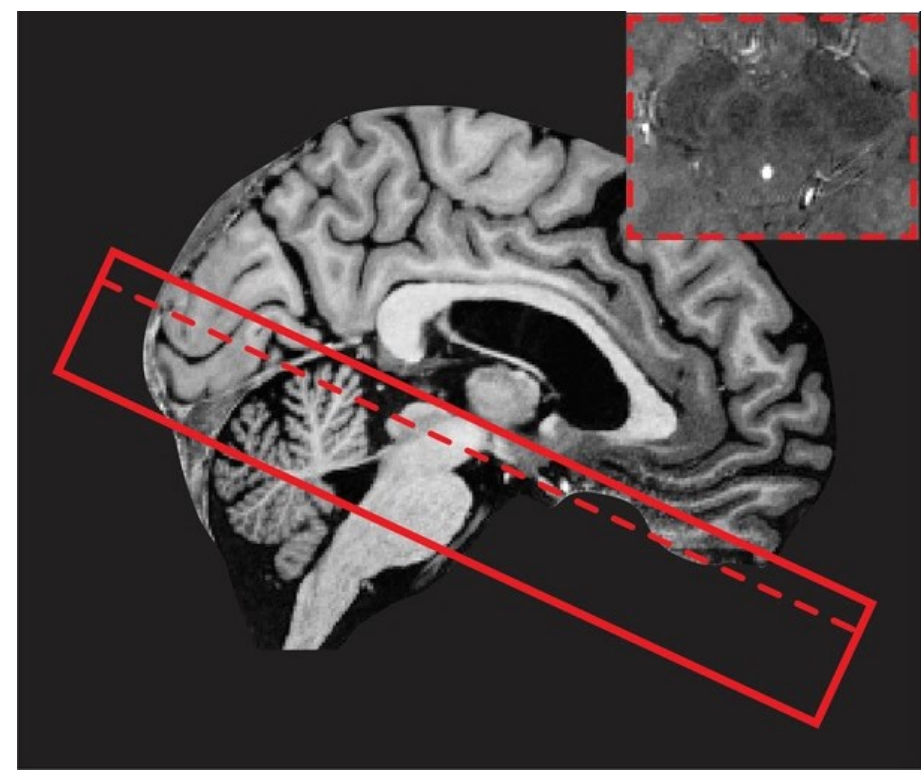

Figure 2. Visualisation of the magnetization transfer-weighted TFL (MTW TFL) field-of-view (FoV) with $3 \mathrm{~cm}$ coverage. The FoV is placed perpendicular to the brainstem. It covers the area between the upper part of the mesencephalon and the lateral recess of the 4th ventricle. Furthermore, an example of the MTW TFL transverse brain stem slice at the substantia nigra is displayed in the right corner. 
Table 2. Technical details of the MRI protocol used for the TRACK-PD study

\begin{tabular}{|c|c|c|c|c|c|c|c|c|c|}
\hline Weighting & Sequence & $\begin{array}{l}\text { TE } \\
\text { (ms) }\end{array}$ & $\begin{array}{l}\text { TR } \\
\text { (ms) }\end{array}$ & $\begin{array}{l}\text { TI } \\
\text { (ms) }\end{array}$ & $\begin{array}{l}\text { Flip angle } \\
\left({ }^{\circ}\right)\end{array}$ & $\begin{array}{l}\text { FoV } \\
(\mathrm{mm})\end{array}$ & $\begin{array}{l}\text { Resolution }\left(\mathrm{mm}^{3}\right) \\
(x-y-z)\end{array}$ & Slices & Orientation \\
\hline \multirow[t]{2}{*}{ T1 } & MP2RAGE & 2.51 & 5000 & 900,2750 & 5 and 3 & 208 & $0.65 \times 0.65 \times 0.65$ & 240 & Sagittal \\
\hline & SA2RAGE & 0.78 & 2400 & 58,1800 & 4 and 10 & 256 & $2.0 \times 2.0 \times 2.0$ & 88 & Sagittal \\
\hline T2* & GRE & $2.49,6.75,13.50,20.75$ & 33 & - & 12 & 204 & $0.5 \times 0.5 \times 0.5$ & 96 & Axial \\
\hline Neuromelanin & MTW TFL & 4.08 & 538 & - & 8 & 192 & $0.4 \times 0.4 \times 0.5$ & 60 & Axial \\
\hline Diffusion & EPI & 60.6 & 7000 & - & 90 & 192 & $1.5 \times 1.5 \times 1.5$ & 80 & Axial \\
\hline fMRI & BOLD & 18.6 & 2000 & - & 70 & 200 & $1.25 \times 1.25 \times 1.25$ & 92 & Axial \\
\hline
\end{tabular}

MP2RAGE Magnetization Prepared 2 Rapid Acquisition Gradient Echoes, SA2RAGE Saturation Prepared with 2 Rapid Gradient Echoes), GRE Gradient Echo, MTW TFL Magnetization transfer weighted TFL, EPI Echo Planar Imaging, BOLD Blood oxygen level dependent, TE Echo time, TR Repetition time, TI Inversion Time, FoV Field of view 
A sample size calculation was based on the effect size of a study that evaluated the dorsal nigral hyperintensity sign in PD and $\mathrm{HC}$ at T2* weighted $7 \mathrm{~T}$ images ${ }^{48}$. Our power analysis with a significance level of $\alpha=0.05$ and power of 0.80 was performed with G*Power (version 3.1.9.4 ${ }^{50}$. Based on this analysis a total sample size of 102 participants, 51 PD and $51 \mathrm{HC}$, is needed to detect a significant difference between these two groups. Due to an expected loss to follow-up of about $10 \%$ for $\mathrm{HC}$, we decided to include $60 \mathrm{HC}$ patients instead of 51. For our secondary objectives we will also perform subgroup analysis for PD patients. To assure a sufficient amount of PD patients for this subgroup analysis, we decided to double the amount of PD patients to 102. Furthermore, the drop-out rate in the PD group is expected to be higher compared to the HCs, due to an increase in disease burden over time. With an expected loss to follow-up of $20 \%$, the PD group should include 130 participants.

\section{Patients and public involvement}

This study has been designed in collaboration with the Dutch Parkinson society. This patient organisation has a scientific advisory board that comments on and contributes to research proposals from a patient's perspective. They will remain involved in the study during the inclusion and follow-up period. Throughout the study, we aim to stay in close contact with all participants. They will be kept informed about the study progress with newsletters and updates on the study website. Moreover, when problems or questions occur, participants can easily contact the study team by e-mail or phone. In this way we intend to minimise the loss to follow-up.

\section{Data collection and management}

The data collected in this study will be stored in a pseudonymized manner. All patient data will be linked to a unique participant number. Two separate databases are in place. Source data of the participants will be kept in a password protected, secure database, which is only accessible by the research team, the health inspector, the data monitor (of the CTCM (Clinical Trial Centre Maastricht))

and members of the medical ethics committee. A second database will be used to store all experimental data and participant numbers. The anonymous data will only be used for research purposes and publications or communications. The handling of personal data will comply with the EU General Data Protection Regulation (GDPR) and the Dutch Act on Implementation of the GDPR. The final study dataset can be accessed only by the research team. Datasets will be made available for other investigators on reasonable request. All study information will be stored for 15 years. Patients who do not give consent to store their information for 15 years, cannot be included in the study. 


\section{Data analysis}

In this study we will analyse both structural and functional MRI data. Raw MRI data will be converted to BIDS NIfTI format for further processing ${ }^{51,52}$. Functional and anatomical MRI images will be pre-processed using MATLAB (MathWorks, version R2018a), the FRMIB Software Library (FSL) ${ }^{53}$ and FreeSurfer (www.freesurfer.net) ${ }^{54}$. Further analysis will be carried out using FSL. All multi-echo GRE, neuromelanin, DWI and functional MRI images will be coregistered with anatomical T1 weighted structural images. Segmentation of the substantia nigra (SN) and locus coeruleus (LC) is manually performed. The subareas of the $\mathrm{SN}$, the pars reticulata and pars compacta ventralis and dorsalis, will also be differentiated. Volumes and signal-to-noise ratio of the SN and LC will be calculated on iron and neuromelanin sensitive images. Both quantitative and qualitative evaluation of the iron sensitive sequences will be performed. For DWI images, diffusion tensor imaging (DTI) will be applied. Furthermore, the fractional anisotropy (FA), radial diffusivity, axial diffusivity and mean diffusivity will be extracted in the SN ROIs. A wholebrain analysis using independent component analysis will be carried out for the functional MRI data. Subsequently, a graph theoretical method is performed to evaluate the topological properties in the whole brain. Finally, predefined ROls will be assessed. A specific interest exists for regions that are part of the default mode network and fronto-parietal network.

Careful visual inspection and quantification of movement will be assessed after each processing step. Both structural and functional MRI differences between HCs and PD patients will be investigated, as well as longitudinal MRI changes in the PD brain.

Clinical and demographic variables will be taken into account as covariates, including handedness, sex, age, disease progression, dopaminergic medication, genetic characteristics, and motor, neuropsychiatric, cognitive and autonomic scores. More specifically, since dopaminergic medication influences brain connectivity patterns both in a linear and non-linear way, the LEDD will be included as a covariate in all fMRI data analysis 55,56 . The numerical variables will be described as means, median, standard deviations and ranges. Categorical variables will be described as frequencies, percentiles, and percentages.

Demographic and disease related variables of the samples at baseline will be compared with Pearson's chi-squared test for categorical variables (non-parametric test) and with the student's t-test and one-way ANOVA for continuous variables. These variables will be included as covariates in the analyses where appropriate.

For our primary objective we will compare structural and functional MRI characteristics between PD patients and HC. This includes iron-sensitive, neuromelanin-sensitive, diffusion weighted and functional sequences. Structural and functional differences will be calculated by using a linear regression model for continuous variables and a logistic 80 
regression model for binary variables. Covariates will be included when needed. The false discovery rate (FDR) method for the correction of multiple comparisons will be applied where appropriate.

After performing the regression analyses for both structural and functional MRI data, we will use a receiver operating characteristics (ROC) method to compute the sensitivity and specificity of the differences found between PD and HC. Furthermore, internal validation will be carried out by using a bootstrap method. In this way we aim to detect the optimal cut-off values for structural and functional differences which distinguish PD patients from HC. Finally, the optimal combination of variables for disease prediction will be assessed by using a logistic regression model. By combining the most important variables, we aim to develop a reliable diagnostic tool with high sensitivity and specificity, that enables us to distinguish PD from HC.

For our secondary objectives, a data-driven cluster analysis will be performed based on the standardized scores of the different variables assessed in our study (motor, genetic, cognitive, autonomic, RBDSQ and neuropsychiatric variables). This enables us to identify different clinical subtypes of PD without a priori assumptions. Structural and functional MRI characteristics will be compared between these different subgroups of PD patients in the same way as described above. Also, longitudinal changes in clinical motor and non-motor symptoms and MRI characteristics will be compared between subgroups by applying a multivariate linear regression model to detect the degree of change for each variable between the PD subgroups.

Furthermore, the longitudinal clinical data (motor, genetic, cognitive, autonomic, RBDSQ and neuropsychiatric variables) of both follow-up moments will be correlated with the MRI characteristics at baseline by using a linear regression model. In this way it can be investigated if the disease course of an individual patient can be predicted based on early MRI-characteristics.

\section{Ethics, safety and dissemination}

The study will be conducted according to the principles of the Declaration of Helsinki (Fortaleza, Brazil, 2013) and in accordance with the Medical Research Involving Human Subjects Act (WMO). All unwanted and harmful outcomes spontaneously reported by the participants, that may or not be related to this study, will be recorded. In case of a serious adverse event, the Ethics committee and relevant authorities will be notified immediately.

This study was approved by the Institutional Review Committee (IRB) of the Maastricht University Medical Centre and written informed consent will be obtained from all participants prior to inclusion. Also, participants are given the choice to provide additional consent for the use of study data and biological specimens in ancillary studies. Before 
consenting, all participants will be extensively informed about the study. During the study patients have the right to withdraw from the study without explanation at any time.

Monitoring of the study will be performed at random moments by employees of CTCM. Those employees are trained and certified in monitoring studies and are not in any way involved in the study.

The results of this study will be shared with clinicians and researchers through scientific conferences and publications in peer-reviewed journals. During the progress of the study preliminary analysis and data validation will be performed, new relevant findings may be published during the study. Furthermore, a summary of the results will also be shared with patients on our web page in an easily comprehensible manner.

\section{Discussion}

The current error-rate for a reliable diagnosis of PD is unacceptable and at this moment it is unclear to what extend changes on MR imaging in PD patients are associated with the clinical deterioration over time. Ultrahigh field imaging has made significant progress in recent years and has a resolution that might replace the requirement for a histologically confirmed diagnosis of PD. This new longitudinal ultra-high field $7 \mathrm{~T} M R I$ study in a PD cohort in which relevant clinical metrics will be obtained for 4 years, will likely improve and change our diagnostic uncertainty in PD.

Several cross-sectional and cohort studies have indicated correlations between MRI characteristics and the clinical symptoms in PD. Most convincingly, a positive correlation between the iron content of the substantia nigra pars compacta and the progression of motor symptoms in PD was demonstrated in several studies 13,14,43,47. In addition, neuromelanin sensitive T1-weighted MRI sequences of the substantia nigra pars compacta may be useful for monitoring motor complications of PD ${ }^{57}$. Also, functional MRI studies demonstrate an altered functional connectivity in PD compared to $\mathrm{HC}$, which is most consistently found in the posterior part of the inferior parietal lobule ${ }^{58}$. Given these developments, the use of multiple MRI modalities and postprocessing techniques such as quantitative susceptibility mapping (QSM) at higher field strengths have the potential to increase diagnostic certainty in PD. However, this has not been tested in a large longitudinal cohort study yet. Furthermore, when high-field MRI research is able to detect whether clinical deterioration or certain PD subtypes are correlated with specific MRI changes, patients can be more accurately informed about their prognosis and treatment options can be adjusted to the individual patient.

In order to correlate MRI changes with clinical progression, monitoring PD symptoms in robust longitudinal clinimetric testing is mandatory ${ }^{19,59}$. Wearable sensors are increasingly used for this purpose ${ }^{60}$. They show promise in detecting tremor ${ }^{61}$, freezing of gait ${ }^{62}$, 
bradykinesia, and dyskinesia ${ }^{63,64}$. Whether wearables will eventually replace commonly used clinical scoring instruments, such as the MDS-UPDRS remains to be seen ${ }^{22}$. The Personalized Parkinson cohort study in The Netherlands is a parallel longitudinal study in which a wearable sensor will be worn for $24 \mathrm{~h}$ a day during a 2 year follow-up period ${ }^{19}$. A subgroup of these participants will also participate in the TRACK-PD study. In the TRACKPD study, wearables are used at baseline and during follow-up visits in addition to classical MDS scoring scales to measure symptom severity. Data from both studies can potentially be combined in the future, providing an even more complete pallet of information to identify patient subgroups in PD.

A potential limitation of this study is the ability of PD patients to lie still in the scanner for $1 \mathrm{~h}$ and the possibility of motion artefacts. However, previous studies have indicated that PD patients are capable and are willing to lie for $1 \mathrm{~h}$ in the scanner for obtaining high quality images ${ }^{65}$. Due to the longitudinal nature of this study there is also a risk of high dropout rates during the follow-up period. By including a significant number of participants, we intend to compensate for the expected loss to follow-up. Moreover, by informing them regularly about the study progress, we aim to stay in close contact with all participants and to reduce the amount of loss to follow-up.

\section{Conclusion}

This is the first longitudinal, observational, ultra-high field MRI study in PD patients. With the TRACK-PD study, an important contribution can be made for the improvement of the current diagnostic process in PD. Moreover, this study will be able to provide valuable information related to the different clinical phenotypes of PD and their correlating MRI characteristics. The long-term aim of this study is to better understand PD and develop new biomarkers for disease progression which may help future new therapy development. Eventually, this may lead to predictive models for individual PD patients and towards personalized medicine in the future. 


\section{References}

1. Al-Radaideh, A. M. \& Rababah, E. M. The role of magnetic resonance imaging in the diagnosis of Parkinson's disease: a review. Clin Imaging 40, 987-996 (2016).

2. Jankovic, J. Parkinson's disease: clinical features and diagnosis. J Neurol Neurosurg Psychiatry 79, 368376 (2008).

3. Postuma, R. B. et al. MDS clinical diagnostic criteria for Parkinson's disease. Mov Disord 30, 1591-1601 (2015).

4. Hughes, A. J., Daniel, S. E., Ben-Shlomo, Y. \& Lees, A. J. The accuracy of diagnosis of parkinsonian syndromes in a specialist movement disorder service. Brain 125, 861-870 (2002).

5. Fereshtehnejad, S.-M., Zeighami, Y., Dagher, A. \& Postuma, R. B. Clinical criteria for subtyping Parkinson's disease: biomarkers and longitudinal progression. Brain 140, 1959-1976 (2017).

6. Konno, T. et al. Comparison of clinical features among Parkinson's disease subtypes: A large retrospective study in a single center. J Neurol Sci 386, 39-45 (2018).

7. Iwaki, H. et al. Genetic risk of Parkinson disease and progression:: An analysis of 13 longitudinal cohorts. Neurol Genet 5, e348 (2019).

8. Thenganatt, M. A. \& Jankovic, J. Parkinson disease subtypes. JAMA Neurol 71, 499-504 (2014).

9. Sieber, B.-A. et al. Prioritized research recommendations from the National Institute of Neurological Disorders and Stroke Parkinson's Disease 2014 conference. Ann Neurol 76, 469-472 (2014).

10. Lehericy, S. et al. The role of high-field magnetic resonance imaging in parkinsonian disorders: Pushing the boundaries forward. Mov Disord 32, 510-525 (2017).

11. Bae, Y. J. et al. Loss of Nigral Hyperintensity on 3 Tesla MRI of Parkinsonism: Comparison With (123) IFP-CIT SPECT. Mov Disord 31, 684-692 (2016).

12. Castellanos, G. et al. Automated neuromelanin imaging as a diagnostic biomarker for Parkinson's disease. Mov Disord 30, 945-952 (2015).

13. Reiter, E. et al. Dorsolateral nigral hyperintensity on 3.0T susceptibility-weighted imaging in neurodegenerative Parkinsonism. Mov Disord 30, 1068-1076 (2015).

14. Schwarz, S. T. et al. The 'Swallow Tail' Appearance of the Healthy Nigrosome - A New Accurate Test of Parkinson's Disease: A Case-Control and Retrospective Cross-Sectional MRI Study at 3T. PLoS One 9, (2014)

15. Filippi, M., Elisabetta, S., Piramide, N. \& Agosta, F. Functional MRI in Idiopathic Parkinson's Disease. Int Rev Neurobiol 141, 439-467 (2018).

16. Wolters, A. F. et al. Resting-state fMRI in Parkinson's disease patients with cognitive impairment: A meta-analysis. Parkinsonism Relat Disord 62, 16-27 (2019).

17. Association, A. Diagnostic and statistical manual of mental disorders (DSM-5 $\left.{ }^{\circledR}\right)$. (2013).

18. Tomlinson, C. L. et al. Systematic review of levodopa dose equivalency reporting in Parkinson's disease. Mov Disord 25, 2649-2653 (2010).

19. Bloem, B. R. et al. The Personalized Parkinson Project: examining disease progression through broad biomarkers in early Parkinson's disease. BMC Neurol 19, 160 (2019).

20. Manniën, J. et al. The Parelsnoer Institute: A National Network of Standardized Clinical Biobanks in the Netherlands. Open Journal of Bioresources 4, 3 (2017).

21. Goetz, C. G. et al. Movement Disorder Society Task Force report on the Hoehn and Yahr staging scale: status and recommendations. Mov Disord 19, 1020-1028 (2004).

22. Goetz, C. G. et al. Movement Disorder Society-sponsored revision of the Unified Parkinson's Disease Rating Scale (MDS-UPDRS): scale presentation and clinimetric testing results. Mov Disord 23, 21292170 (2008).

23. Heijmans, M. et al. Monitoring Parkinson's disease symptoms during daily life: a feasibility study. NPJ Parkinsons Dis 5, 21 (2019). 
24. Visser, M., Marinus, J., Stiggelbout, A. M. \& Van Hilten, J. J. Assessment of autonomic dysfunction in Parkinson's disease: the SCOPA-AUT. Mov Disord 19, 1306-1312 (2004).

25. Palavra, N. C., Naismith, S. L. \& Lewis, S. J. G. Mild cognitive impairment in Parkinson's disease: a review of current concepts. Neurol Res Int 2013, 576091 (2013).

26. Gladsjo, J. A. et al. Norms for letter and category fluency: demographic corrections for age, education, and ethnicity. Assessment 6, 147-178 (1999).

27. Vakil, E. \& Blachstein, H. Rey Auditory-Verbal Learning Test: structure analysis. J Clin Psychol 49, 883890 (1993).

28. Benton, A. L., Varney, N. R. \& Hamsher, K. D. Visuospatial judgment. A clinical test. Arch Neurol 35, 364-367 (1978).

29. Smith, A. Symbol Digit Modalities Test (SDMT) manual. (1982).

30. Wechsler, D. Wechsler adult intelligence scale. (2008).

31. Visser, M., Leentjens, A. F. G., Marinus, J., Stiggelbout, A. M. \& van Hilten, J. J. Reliability and validity of the Beck depression inventory in patients with Parkinson's disease. Mov Disord 21, 668-672 (2006).

32. Leentjens, A. F. G. et al. The Parkinson Anxiety Scale (PAS): development and validation of a new anxiety scale. Mov Disord 29, 1035-1043 (2014).

33. Probst, C. C. et al. Validation of the questionnaire for impulsive-compulsive disorders in Parkinson's disease (QUIP) and the QUIP-rating scale in a German speaking sample. J Neurol 261, 936-942 (2014).

34. Luo, N. et al. Determination of the longitudinal validity and minimally important difference of the 8item Parkinson's Disease Questionnaire (PDQ-8). Mov Disord 24, 183-187 (2009).

35. Stiasny-Kolster, K. et al. The REM sleep behavior disorder screening questionnaire--a new diagnostic instrument. Mov Disord 22, 2386-2393 (2007).

36. Blauwendraat, C., Nalls, M. A. \& Singleton, A. B. The genetic architecture of Parkinson's disease. Lancet Neurol 19, 170-178 (2020).

37. Jakubowski, J. L. \& Labrie, V. Epigenetic Biomarkers for Parkinson's Disease: From Diagnostics to Therapeutics. J Parkinsons Dis 7, 1-12 (2017).

38. Teeuwisse, W. M., Brink, W. M. \& Webb, A. G. Quantitative assessment of the effects of highpermittivity pads in 7 Tesla MRI of the brain. Magn Reson Med 67, 1285-1293 (2012).

39. Eggenschwiler, F., Kober, T., Magill, A. W., Gruetter, R. \& Marques, J. P. SA2RAGE: a new sequence for fast B1+ -mapping. Magn Reson Med 67, 1609-1619 (2012).

40. Priovoulos, N. et al. High-resolution in vivo imaging of human locus coeruleus by magnetization transfer MRI at 3T and 7T. Neuroimage 168, 427-436 (2018).

41. Ofori, E. et al. Longitudinal changes in free-water within the substantia nigra of Parkinson's disease. Brain 138, 2322-2331 (2015).

42. Ulla, M. et al. Is R2* a new MRI biomarker for the progression of Parkinson's disease? A longitudinal follow-up. PLoS One 8, e57904 (2013).

43. Wieler, M., Gee, M. \& Martin, W. R. W. Longitudinal midbrain changes in early Parkinson's disease: iron content estimated from R2*/MRI. Parkinsonism Relat Disord 21, 179-183 (2015).

44. Mak, E. et al. Baseline and longitudinal grey matter changes in newly diagnosed Parkinson's disease: ICICLE-PD study. Brain 138, 2974-2986 (2015).

45. Alkemade, A. et al. Comparison of T2*-weighted and QSM contrasts in Parkinson's disease to visualize the STN with MRI. PLoS One 12, e0176130 (2017).

46. Cosottini, M. et al. MR imaging of the substantia nigra at $7 \mathrm{~T}$ enables diagnosis of Parkinson disease. Radiology 271, 831-838 (2014).

47. Kim, J.-M. et al. Loss of substantia nigra hyperintensity on 7 Tesla MRI of Parkinson's disease, multiple system atrophy, and progressive supranuclear palsy. Parkinsonism Relat Disord 26, 47-54 (2016).

48. Pyatigorskaya, N. et al. Comparative Study of MRI Biomarkers in the Substantia Nigra to Discriminate Idiopathic Parkinson Disease. AJNR Am J Neuroradiol 39, 1460-1467 (2018). 
49. Schmidt, M. A. et al. Ultra high-field SWI of the substantia nigra at 7T: reliability and consistency of the swallow-tail sign. BMC Neurol 17, 194 (2017).

50. Faul, F., Erdfelder, E., Lang, A.-G. \& Buchner, A. G*Power 3: a flexible statistical power analysis program for the social, behavioral, and biomedical sciences. Behav Res Methods 39, 175-191 (2007).

51. Gorgolewski, K. J. et al. BIDS apps: Improving ease of use, accessibility, and reproducibility of neuroimaging data analysis methods. PLoS Comput Biol 13, e1005209 (2017).

52. Li, X., Morgan, P. S., Ashburner, J., Smith, J. \& Rorden, C. The first step for neuroimaging data analysis: DICOM to NIfTI conversion. J Neurosci Methods 264, 47-56 (2016).

53. Jenkinson, M., Beckmann, C. F., Behrens, T. E. J., Woolrich, M. W. \& Smith, S. M. FSL. Neuroimage 62, 782-790 (2012).

54. Dale, A. M., Fischl, B. \& Sereno, M. I. Cortical surface-based analysis. I. Segmentation and surface reconstruction. Neuroimage 9, 179-194 (1999).

55. Cole, D. M. et al. Differential and distributed effects of dopamine neuromodulations on resting-state network connectivity. Neuroimage 78, 59-67 (2013).

56. Dang, L. C., O'Neil, J. P. \& Jagust, W. J. Dopamine supports coupling of attention-related networks. J Neurosci 32, 9582-9587 (2012).

57. Hatano, T. et al. Neuromelanin MRI is useful for monitoring motor complications in Parkinson's and PARK2 disease. J Neural Transm (Vienna) 124, 407-415 (2017).

58. Tahmasian, M. et al. Resting-state functional reorganization in Parkinson's disease: An activation likelihood estimation meta-analysis. Cortex 92, 119-138 (2017).

59. Parkinson Progression Marker Initiative. The Parkinson Progression Marker Initiative (PPMI). Prog Neurobiol 95, 629-635 (2011).

60. Sánchez-Ferro, Á. et al. New methods for the assessment of Parkinson's disease (2005 to 2015): A systematic review. Mov Disord 31, 1283-1292 (2016).

61. Delrobaei, M. et al. Towards remote monitoring of Parkinson's disease tremor using wearable motion capture systems. J Neurol Sci 384, 38-45 (2018).

62. Rodríguez-Martín, D. et al. Home detection of freezing of gait using support vector machines through a single waist-worn triaxial accelerometer. PLoS One 12, e0171764 (2017).

63. Delrobaei, M., Baktash, N., Gilmore, G., Mclsaac, K. \& Jog, M. Using Wearable Technology to Generate Objective Parkinson's Disease Dyskinesia Severity Score: Possibilities for Home Monitoring. IEEE Trans Neural Syst Rehabil Eng 25, 1853-1863 (2017).

64. Griffiths, R. I. et al. Automated assessment of bradykinesia and dyskinesia in Parkinson's disease. J Parkinsons Dis 2, 47-55 (2012).

65. Plantinga, B. R. et al. Individualized parcellation of the subthalamic nucleus in patients with Parkinson's disease with 7T MRI. Neuroimage 168, 403-411 (2018). 


\section{Chapter 6}

Comparison of olfactory tract diffusion measures between early Parkinson's disease patients and healthy controls using ultrahigh field MRI

M. Heijmans, A.F. Wolters, Y. Temel, M.L. Kuijf, S. Michielse Submitted. 


\begin{abstract}
Background

MRI is a valuable method to assist in the diagnostic work-up of Parkinson's Disease (PD). The olfactory tract has been proposed as a potential MRI biomarker target for discriminating PD patients from healthy controls. This study aims to further investigate whether diffusion measures of the olfactory tract differ between early PD patients and healthy controls.
\end{abstract}

\title{
Methods
}

In total, 62 PD patients and 27 healthy controls were evaluated and a whole brain 7T diffusion weighted image scan was acquired. PD patients were diagnosed up to three years before inclusion. Participant inclusion did not depend on the ability to smell and to detect odours. Manual seed regions of interest were drawn in the bilateral olfactory tract region. Next, tractography of the olfactory tract was performed using a deterministic streamlines algorithm based on spherical deconvolution. Diffusion measures (fractional anisotropy and mean- radial- and axial diffusivity) of the generated streamlines were calculated.

\section{Results}

Diffusion measures of the olfactory tract did not differ between PD patients and healthy controls. Axial diffusivity values were higher in PD patients with REM-sleep behaviour disorder compared to those without. Age positively correlated with mean and axial diffusivity measures of the olfactory tract in PD patients but not in healthy controls. PD symptom scores did not correlate with the diffusion measures of the olfactory tract.

\section{Conclusion}

This study showed that fiber tracking of the olfactory tract was feasible in both early PD and healthy controls using 7T diffusion weighted imaging data. However, 7T MRI diffusion measures of the olfactory tract are not useful as an early clinical biomarker for PD. Future work is needed to clarify the role of other olfactory tract measurements as a biomarker for PD and its different subgroups.

\section{Keywords}

Parkinson's disease; olfactory tract; diffusion weighted imaging; biomarker; ultra-high field imaging 


\section{Introduction}

Parkinson's disease (PD) is a neurodegenerative disorder, often recognized by clinicians when motor symptoms like bradykinesia, rigidity and tremor become apparent. In current clinical practice, the diagnosis PD is based on the assessment of symptoms and their course over time according to clinical criteria ${ }^{1}$. However, discriminating PD from other parkinsonian syndromes can be difficult as is illustrated by the error rate of $24 \%$ for a clinical diagnosis of PD, even in specialized movement disorders centres ${ }^{2}$. The diagnostic challenge is in part due to PD being a heterogeneous disease with variation in clinical course, response to treatment, genetic background and the influence of environmental factors. The gold standard for diagnosis remains a neuropathological confirmation of dopaminergic neuronal cell loss. Interestingly, recent advances in neuroimaging have led to the development of several new MRI technologies and to optimism for new useful noninvasive biomarkers for diagnosing PD.

MRI is a valuable method to assist in the diagnostic work-up of PD. Most prominently described in recent years as potential biomarkers to discriminate PD patients from healthy controls ( $\mathrm{HC}$ ) are the signal loss of the nigrosome- 1 area on iron sensitive images and reduced signal intensity of the substantia nigra on neuromelanin sensitive images ${ }^{3-6}$. However, limitations of previous studies that investigated these potential MRI biomarkers are small sample sizes, low imaging resolution and the inclusion of already advancedstaged PD patients in cohort studies. Moreover, most research utilized MRI scanners with a field strength below 7T, with 3T being commonly applied in studies. With the application of various 7T MRI sequences, like iron sensitive scans, neuromelanin sensitive scans and diffusion weighted imaging (DWI) scans, diagnostic confidence may improve due to increased spatial resolution and a higher signal to noise ratio as a result of ultra-high field imaging 5,7 .

DWI is a MRI technique which enables the measurement of the random Brownian motion of water molecules within the brain ${ }^{8}$. This movement can be quantified via Diffusion Tensor Imaging (DTI), which helps to better understand the underlying white matter neurobiology on the macroscopic level. Commonly used measures are the fractional anisotropy (FA), which quantifies the preferred diffusion direction of water molecules and fibre mixture, the mean diffusivity (MD), which represents the diffusion magnitude, the radial diffusivity $(R D)$, which is perpendicular to axons and reflects myelination, and the axial diffusivity (AD), which is parallel to axons and reflects organization ${ }^{9,10}$. DTI can be used for the reconstruction of fibre tracts, which allows to perform a virtual dissection of white matter on the voxel level.

The decreased ability to smell and to detect odours is one of the classical early symptoms of PD. The pathological mechanism is poorly understood but possibly involves neurodegeneration of the olfactory tract (OT) ${ }^{1}$. In fact, from a neuropathological point of 
view, the OT and olfactory bulb are often recognized as one of the first structures affected, according to the well accepted Braak stadia of PD pathology ${ }^{11}$. Previously, a meta-analysis highlighted that the OT could potentially serve as a biomarker for PD ${ }^{12}$. However, previous work studying diffusion measures of the OT or olfactory areas showed varying results. Based on DTI subregion analyses in PD patients and HC, lower FA was reported in the anterior olfactory regions of the PD patients without overlapping distributions between groups ${ }^{13}$. Furthermore, the MD of the OT was higher in PD patients compared to $\mathrm{HC}$ and correlated positively with OFF-medication motor scores ${ }^{14}$. In addition, AD was lower in the white matter adjacent to the olfactory sulcus, which can be attributed to potential axonal degeneration ${ }^{15}$. When comparing PD patients with $\mathrm{HC}$, it was shown that both FA and tract volume of the OT were decreased in PD ${ }^{16}$

In previous studies, the included PD groups had a significantly decreased ability to smell and to detect odours compared to the HC groups ${ }^{13-16}$. Not all PD patients experience hyposmia at the time of diagnosis. Therefore, the reported findings should be replicated in an unselected group of early PD patients, without taking into account their ability to smell and to detect odours. Only then it can be determined whether DWI measures of the OT may be able to serve as an early biomarker for PD.

This study aims to further investigate whether DWI measures of the OT differ between an unselected group of early PD patients and HC. This report is part of the TRACK-PD study, which is an observational ultra-high field imaging study aiming to provide more information on diagnosing PD and its subtypes ${ }^{7}$. We hypothesize that FA values in the OT will be decreased in PD patients compared to $\mathrm{HC}$, while MD values will be increased in PD patients. Additionally, DWI measures of the OT will be compared between HC, a less severe PD group and a more severe PD group (based on Hoehn and Yahr score), and between PD groups with and without rapid eye movement (REM) sleep behaviour disorder, since olfactory dysfunction is strongly associated with this ${ }^{17}$. Last, the association between age and diffusion measures of the OT and PD symptom scores and diffusion measures of the OT will be explored.

\section{Materials and Methods}

\section{Participants}

Data collection was performed between July 2019 and December 2020 as part of the TRACK-PD study. The inclusion procedure, in- and exclusion criteria, and the study procedure are described elsewhere ${ }^{7}$. In short, patients were diagnosed with PD maximally three years before the first testing day and were excluded when diagnosed with neurodegenerative diseases other than PD. Overall, participants were excluded when they scored $<24$ points on the Montreal Cognitive Assessment [ $\mathrm{MoCa}{ }^{18}$ ] or when they had any contraindications (claustrophobia or ferromagnetic implants) for the 7T MRI scan. During 90 
the testing day, patients took their prescribed PD medication. The TRACK-PD study was approved by the Institutional Review Committee (IRB) of the Maastricht University Medical Centre and written informed consent was obtained from all participants.

\section{Clinical assessments}

During the testing day motor functions were assessed by trained and certified investigators using the unified Parkinson's Disease rating scale (MDS-UPDRS) and the Hoehn an Yahr scale. The MDS-UPDRS consists of four parts, including a motor evaluation (MDS-UPDRSIII)

19. The Hoehn and Yahr scale is used to estimate the global disease stage of PD patients ${ }^{20}$. Autonomic functions were assessed using the Scales for Outcomes in Parkinson's Disease - Autonomic dysfunction questionnaire (SCOPA-AUT) ${ }^{21}$. Symptoms related to REM-sleep behaviour disorders were assessed using the REM-sleep behaviour disorder screening questionnaire (RBDSQ) ${ }^{22}$.

\section{MRI acquisition}

Participants were scanned in a 7T MRI scanner (Magnetom, Siemens, Erlangen, Germany) equipped with a Nova Medical 32-channel head coil. PD patients were scanned in the ONmedication state for practical reasons such as a lower chance of motion artifacts by OFFrelated tremors (if present). When possible, dielectric pads were applied to enhance the signal in especially the temporal brain regions ${ }^{23}$. During the scan session, participants had the possibility to watch a movie projected on a screen, which was visible via a mirror.

The MRI protocol of the TRACK-PD study was described elsewhere ${ }^{7}$. The current paper has a focus on the DWI scans. First a localizer sequence was acquired for optimal planning, and B0 and B1 mapping and shimming were used to correct for field inhomogeneities. A whole brain DWI scan was acquired using an echo-planar imaging (EPI) sequence, along 66 random directions with an average B-value of $2000 \mathrm{~s} / \mathrm{mm}^{2}$ mixed with six B0-volumes. One B0-volume and five DWI volumes were acquired with an opposite phase encoding direction. Technical details of the DWI scan included; TE $=60.6 \mathrm{~ms}, \mathrm{TR}=7000 \mathrm{~ms}$, flip angle $=90$ degrees, FOV $=192 \times 192 \mathrm{~mm}$, resolution $=1.5 \mathrm{~mm}$ isotropic, number of slices $=80$, orientation = interleaved axial, GeneRalized Autocalibrating Partial Parallel Acquisition (GRAPPA) ${ }^{24}$ acceleration factor $=3$, and acquisition time $=9: 48$ minutes.

\section{MRI preprocessing}

After conversion of the raw DICOM data to nifti format ${ }^{25}$, DWI scans were denoised and potential Gibbs ringing artifacts were removed using MRtrix version $3^{26-28}$. Following this, the DWI scans were corrected for susceptibility induced distortions using topup, eddy currents and subject motion 29-31, and b-matrix reorientation was performed using software from the FMRIB software library of FSL 5.0 (http://www.fmrib.ox.ac.uk/fsl). After this the generated mask was manually enlarged to make sure the area covering the OT was 
included. Data quality control was performed by visual inspection and by quantification of the mean displacement values per volume based on the eddy current correction routine ${ }^{32}$.

\section{OT tractography}

Seed regions of interest (ROI) were manually drawn by two raters ( $\mathrm{MH}$ and $\mathrm{AW}$ ) blinded to the clinical status of the participants. For each dataset, the average image of the unwarped and movement corrected BO volumes was viewed. Next, an empty mask was created and seed ROls were drawn according to the following guidelines; 1 ) For each participant, two axial slices of the average $\mathrm{BO}$ image were selected in which the OT was best visible (Figure 1A). 2) Within these slices, five conjoined squares of $2 \times 2$ voxels were drawn for both the left and right $\mathrm{OT}$, resulting in 40 voxels per slice and 80 voxels per participant (Figure $1 \mathrm{~B}$ ).

Tractography of the OT was performed using MRtrix3. First, the response function was estimated with the constrained spherical deconvolution algorithm ${ }^{33}$ and the fiber orientation distribution ${ }^{34}$ was estimated. Next, streamline tractography was performed utilizing the drawn seed ROI as seed and with the deterministic streamlines algorithm based on spherical deconvolution ${ }^{35}$ (angle threshold $=30$ degree, minimum track length $=$ $5 \mathrm{~mm}$, seeding direction = anterior-posterior). An example of the tractography results are shown in Figure $1 C$. Finally, tensors were fitted ${ }^{36}$ and metrics were calculated in MRTrix3 to generate $F A, M D, A D$ and $R D$ maps. The total number of tracts, mean $F A, M D, A D$ and $\mathrm{RD}$ values of the generated streamlines were calculated. The average diffusion measures of both raters were used for further analysis.

\section{Statistical analysis}

Statistical analysis was performed using JASP 0.14.1.0 and Rstudio 4.0.4. Demographic variables were compared between groups with Pearson's chi-squared test for categorical variables and the student's t-test for continuous variables. Non-parametric tests were used in case the assumption of normality was violated. Inter rater reliability of the two ROI raters was assessed using the Intraclass Correlation Coefficient (ICC) ${ }^{37}$. Any differences in diffusion measures of the OT between groups were tested utilizing ANCOVA (adjusting for possible demographic variables between groups). Correlations between diffusion measures of the OT and age and correlations between diffusion measures of the OT and PD symptom scores (MDS-UPDRSIII score, disease duration, SCOPA-AUT and RBDSQ) were assessed using Spearman correlation. Bonferroni corrections were applied to correct for multiple comparisons, leading to a significance level of 0.0125 for the correlation analyses. 


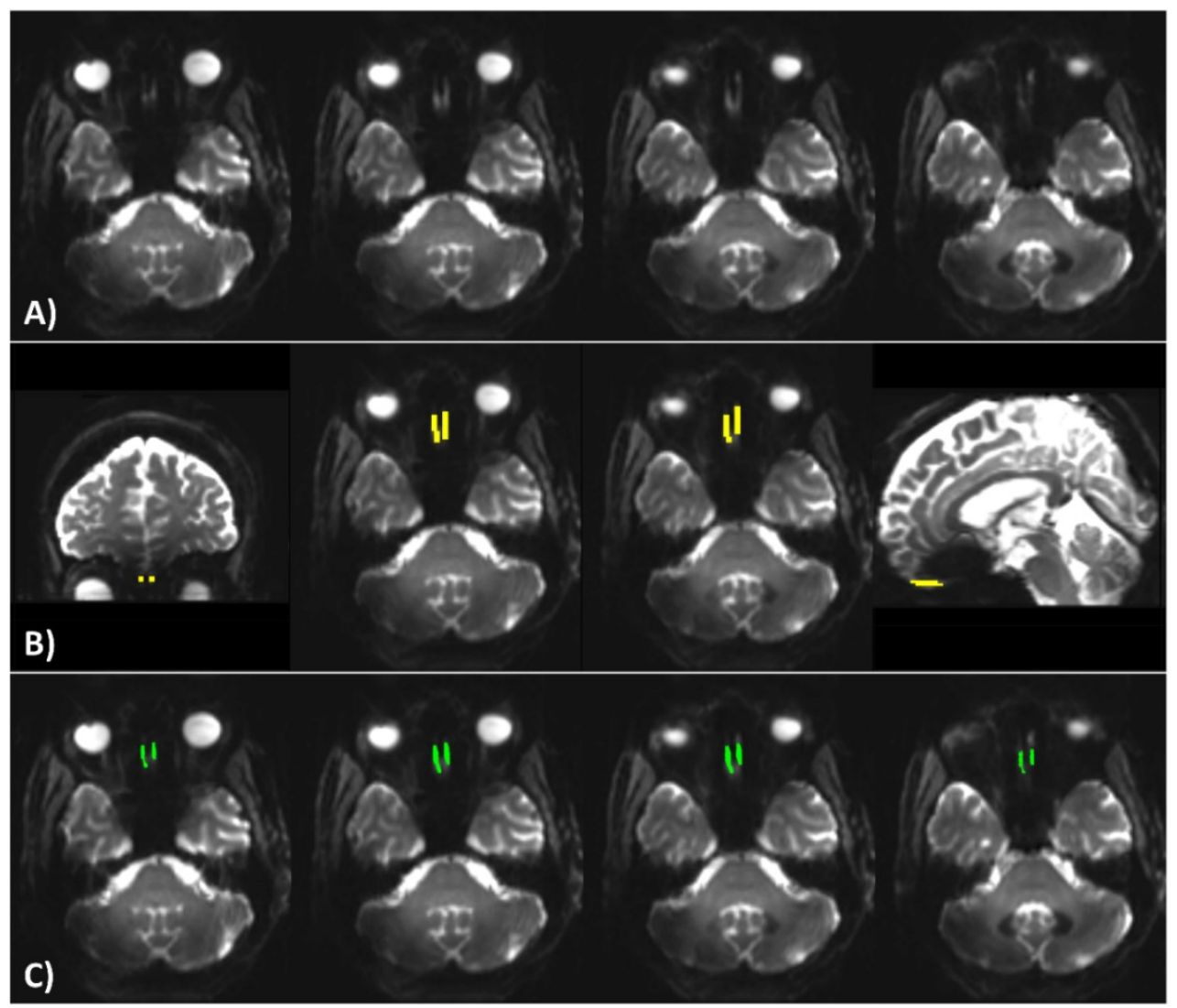

Figure 1. A) Four axial slices of the average corrected B0 volumes. The two middle images were selected as the ones having the best visible olfactory tract. B) Coronal, axial, and sagittal view of the seed ROI representing the olfactory tract area. C) The same four axial slices as shown in A, showing the olfactory tract tractography results. Images are in radiological orientation.

\section{Results}

\section{MRI quality control}

Data quality control resulted in the exclusion of six datasets (three PD and three $H C$ ), resulting in a total of $62 \mathrm{PD}$ and $27 \mathrm{HC}$ participants. Two datasets were excluded since they contained multiple volumes which had a mean displacement larger than the voxel size (1.5 $\mathrm{mm}$ ). Four datasets were excluded since the field of view did not cover the OT area.

\section{Demographics}

Participant characteristics are shown in Table 1. No significant differences were found for age and MoCa scores between the PD and HC groups ( $p=0.080$ and $p=0.698$ respectively). A significant association was found between sex and group $\left(\chi^{2}(1)=4.253, p=0.039\right)$, indicating a difference in sex distribution between the groups. Based on this finding, further 
analyses were corrected for sex. SCOPA-AUT and RBDSQ scores were higher for the PD patients compared to $\mathrm{HC}(\mathrm{p}<0.001$ and $\mathrm{p}=0.007$ respectively). The Hoehn and Yahr score showed that most patients were in an early stage of the disease.

Table 1. Participant characteristics

\begin{tabular}{|c|c|c|c|}
\hline & PD patients $(n=62)$ & Healthy controls $(n=27)$ & P value \\
\hline Male/female, $n$ & $44 / 18$ & $13 / 14$ & 0.039 \\
\hline Age, year & $62.6(9.3)$ & $58.9(8.9)$ & 0.080 \\
\hline MoCa & $27.8(1.6)$ & $27.7(1.5)$ & 0.698 \\
\hline SCOPA-AUT & $14.6(6.9)$ & $7.5(4.7)$ & $<0.001$ \\
\hline RBDSQ & $4.1(4.3)$ & $1.6(2.9)$ & 0.006 \\
\hline Time since PD diagnosis, months & $18.5(9.3)$ & & \\
\hline Levodopa Equivalent Daily Dose & $373.8(233.7)$ & & \\
\hline MDS-UPDRSIII & $18.5(7.2)$ & & \\
\hline Hoehn and Yahr stage, $n$ & $\begin{array}{l}\text { Stage 1: } 35 \\
\text { Stage 2: } 24 \\
\text { Stage 3: } 3 \\
\text { Stage 4: } 0\end{array}$ & & \\
\hline
\end{tabular}

\section{Inter rater reliability}

The diffusion measures extracted from the tracts of the two ROI raters showed a good (FA $I C C=0.87, \mathrm{RD} I C C=0.86, \mathrm{AD} I C C=0.83$ ) and excellent (MD ICC $=0.91)$ inter rater reliability. The ICC of the two ROI raters showed a moderate reliability when looking at the number of tracts of both raters (ICC $=0.57$ ). This number is dependent on the exact placement of the ROIs and therefore it can be expected that the reliability is neither good or excellent.

\section{Group comparison of diffusion measures of the OT}

The number of tracts did not significantly differ between the PD $($ mean $=410.9$, std $=118.1$ ) and the HC group (mean $=384.6$, std $=83.4$ ). Diffusion measures of the OT were compared between the PD and $\mathrm{HC}$ group while adjusting for the difference in sex distribution between groups. There was no significant difference of $F A, M D, R D$, and $A D$ values of the OT between PD and HC (Figure 2, Table 2).

Diffusion measures of the OT were compared between the HC group, PD patients with a Hoehn and Yahr score of $1(n=35)$ and PD patients with a Hoehn and Yahr score of 2 or higher $(n=27)$. There was no significant difference of $F A, M D, R D$ and $A D$ values between the three groups (Supplementary Figure 1, Supplementary Table 1). 

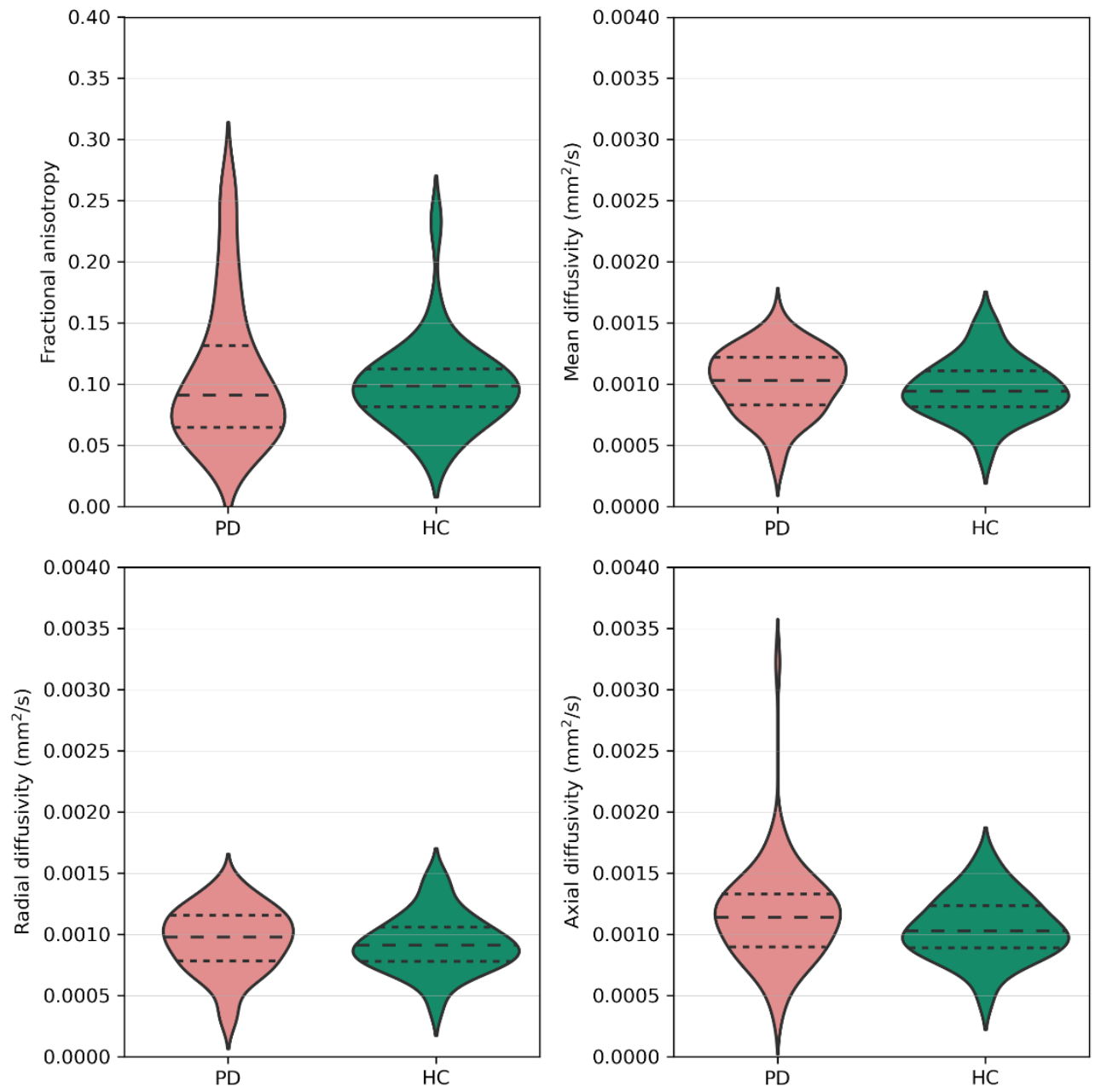

Figure 2. Fractional anisotropy, mean diffusivity, radial diffusivity and axial diffusivity values of the olfactory tract of Parkinson's disease patients (PD) and healthy controls (HC). Dashed lines represent the quartiles of the distribution.

Table 2. Diffusion measures of the olfactory tract

\begin{tabular}{llll} 
& PD patients $(\mathbf{n}=62)$ & Healthy controls $(\mathbf{n}=\mathbf{2 7})$ & P value \\
\hline FA & $0.108446(0.058977)$ & $0.099815(0.036433)$ & 0.741 \\
\hline MD $\left(\mathrm{mm}^{2} / \mathrm{s}\right)$ & $0.001019(0.000262)$ & $0.000966(0.000236)$ & 0.971 \\
$\mathbf{R D}\left(\mathrm{mm}^{2} / \mathrm{s}\right)$ & $0.000951(0.000262)$ & $0.000923(0.000230)$ & 0.644 \\
\hline AD $\left(\mathrm{mm}^{2} / \mathrm{s}\right)$ & $0.001154(0.000403)$ & $0.001051(0.000253)$ & 0.157
\end{tabular}

Data are presented as mean (std). $F A=$ fractional anisotropy, $M D=$ mean diffusivity, $R D=$ radial diffusivity, $A D=$ axial diffusivity 
Next, diffusion measures of the OT were compared between PD patients with (RBDSQ $>0$, $n=34$ ) and without (RBDSQ $=0, n=28$ ) REM sleep behaviour disorder. AD values were significantly higher $(p=0.0377)$ for the PD group with REM sleep behaviour disorder (mean $=0.001241$, std $=0.000448$ ) compared to the PD group without REM sleep behaviour disorder $($ mean $=0.001049$, std $=0.000317$ ) (Figure 3 ). There were no significant differences in FA, MD and RD values (Supplementary Figure 2, Supplementary Table 2).

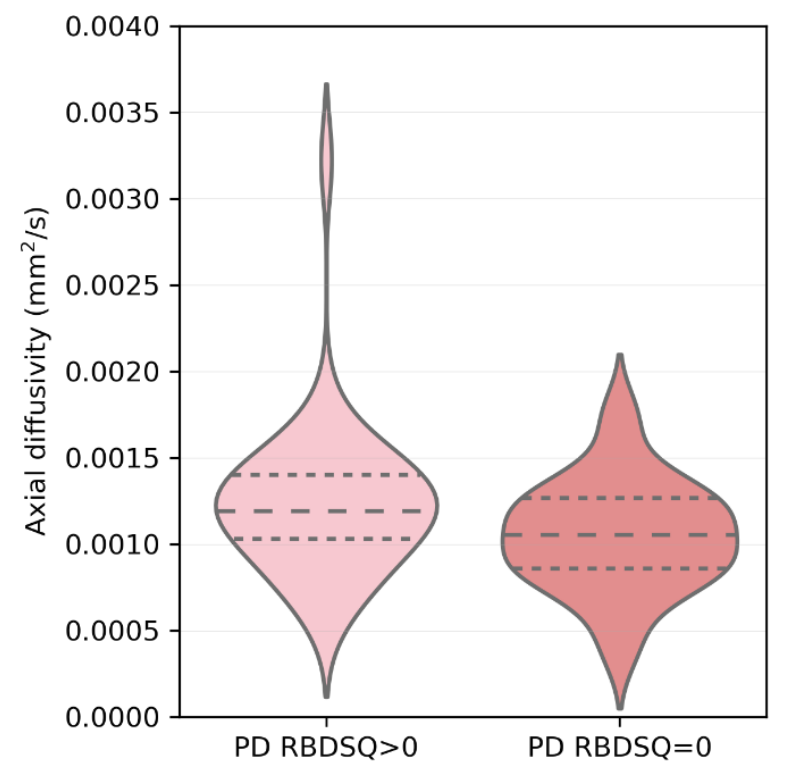

Figure 3. Axial diffusivity values of the olfactory tract of Parkinson's disease patients with REM sleep behaviour disorder (PD RBDSQ > 0) and Parkinson's disease patients without REM sleep behaviour disorder (PD RBDSQ $=0$ ). Dashed lines represent the quartiles of the distribution.

\section{Clinical correlations of diffusion measures of the OT}

For the total group, significant positive correlations were found between $M D$ and age ( $r=0.322, p=0.002)$ and $A D$ and age $(r=0.350, p=0.001)$, but not between $F A, R D$ and age. When $P D$ and $\mathrm{HC}$ groups were compared separately regarding the association of $M D$ and $A D$ with age, significant positive correlations were found between $M D(r=0.381, p=0.002)$ and $A D(r=0.405, p=0.001)$ with age for the PD group. In contrast, no significant correlations were found for the $\mathrm{HC}$ group, suggesting that $M D$ and $A D$ values in PD patients increase over time, while this is not the case in the HC group (Figure 4). In the PD group, MDSUPDRSIII (ON-medication), disease duration, SCOPA-AUT, and RBDSQ scores did not significantly correlate with FA, MD, RD and AD scores of the OT ( $p>0.0125)$. 

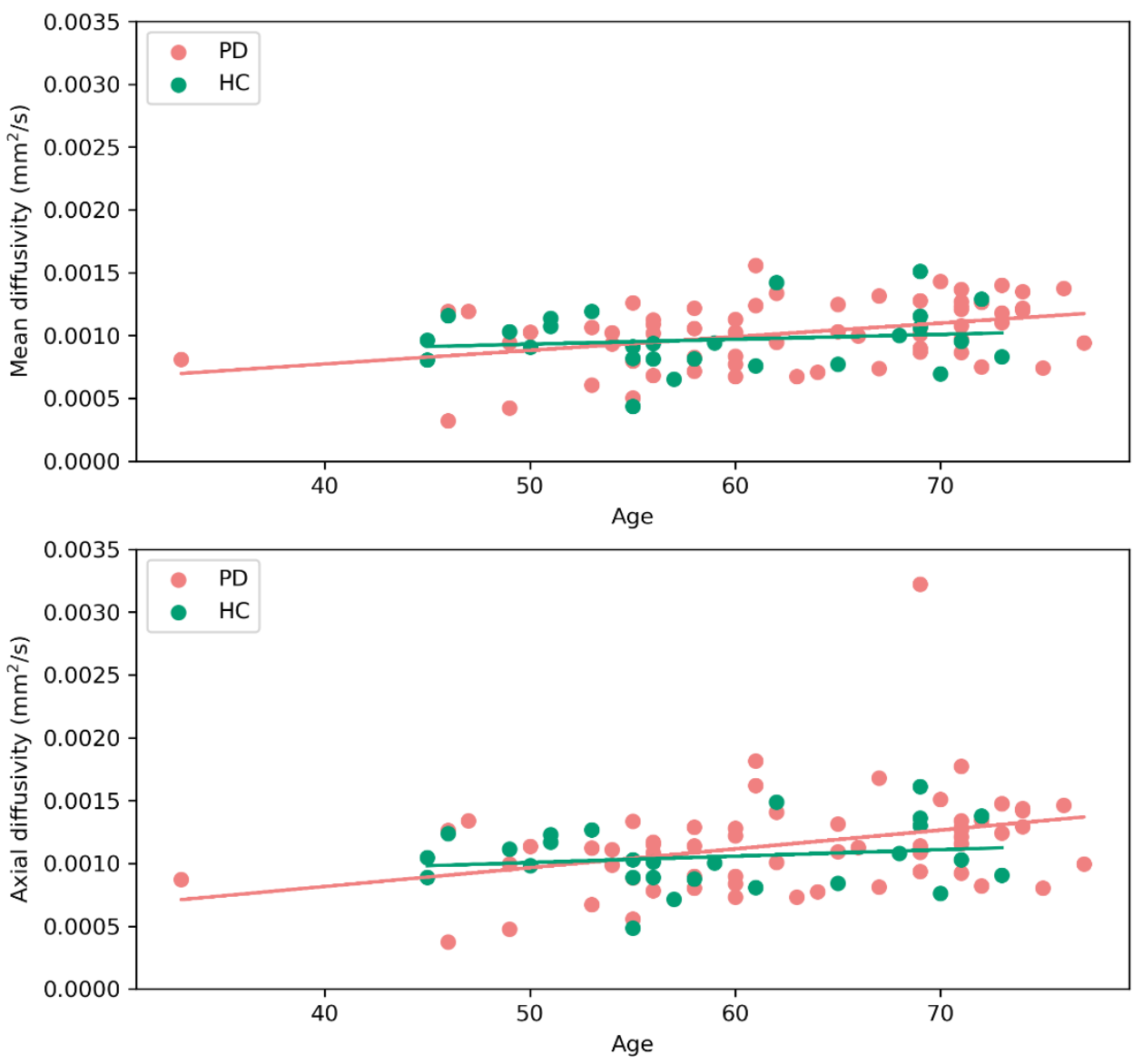

Figure 4. Correlation between mean diffusivity and age per group, and correlation between axial diffusivity and age per group. For only the Parkinson's disease (PD) group significant correlations were found between age and mean diffusivity $(r=0.381, p=0.002)$ and between age and axial diffusivity $(r=0.405, p=0.001)$.

\section{Discussion}

This is the first study which shows feasible and reliable fiber tracking of the OT in both early PD and HC using 7T DWI data. We showed that FA, MD, RD, and AD measures of the OT did not differ between early PD patients and HC. These findings are not in line with our hypothesis, in which we hypothesized that FA values of the OT would be decreased in PD, and MD values of the OT would be increased in PD compared to HC. Furthermore, diffusion measures of the OT were compared between $\mathrm{HC}$, a less severely and a more severely affected PD group, and between PD groups with and without REM sleep behaviour disorder. $A D$ values were higher for PD patients with REM sleep behaviour disorder compared to the PD group without these symptoms. Additionally, a significant positive correlation was found for age with MD and AD within the PD subgroup, but not within the 
HC group. No significant correlations between MDS-UPDRSIII (ON-medication state), disease duration, SCOPA-AUT, and RBDSQ scores and diffusion measures of the OT were found for the PD group.

Previous work studying diffusion measures of the OT or olfactory areas showed varying results. For instance, the MD in the OT region was higher in PD compared to $\mathrm{HC}$ and a positive correlation between MD and MDS-UPDRSIII motor score (OFF-medication) was found ${ }^{14}$. In addition, the FA of anterior olfactory regions and the OT was found to be decreased in PD patients ${ }^{13,16}$. FA decrease and MD increase are thought to be a result of neuronal and myelin damage, which lead to a depletion of restricting barriers to the motion of water molecules. Combined FA decreases and MD increases were observed in brain areas known to be affected by neurodegenerative processes in patients with for example Alzheimer's disease ${ }^{38}$. However, no study so far showed both a decrease in FA and an increase in MD in the OT of PD patients compared to controls.

The correlation found in this study between age and MD suggests that aging might play an important role in the DWI alternations found in the OT. Since the positive correlation of the MD with age was found only within our PD subgroup, but not within our age-matched $\mathrm{HC}$ group, it could be suggested that aging is accelerated within the PD brain compared to the $\mathrm{HC}$ brain. Another recent tractography study however showed a negative correlation between FA values of the OT and age for only the HC group and not for the hyposmic PD group, suggesting that hyposmic PD patients show a more constant and already early decreased FA in the OT, while the FA of the HC group decreases with age ${ }^{16}$. This would infer that for FA in the OT, differences should already be present when comparing groups, hyposmic PD with HC, of a relatively low mean age. Future work comparing PD and HC groups of varying ages should be performed to replicate findings and to unravel how the correlations between FA and MD of the OT and age should be interpreted.

The positive correlation between age and $A D$ values for only the PD group is interesting. Based on previous work ${ }^{15}$, it is expected that $A D$ values would decrease with age, and especially in the PD group, since $A D$ values can be attributed to axonal degeneration. The higher $A D$ values in the PD group with REM sleep behaviour disorder compared to the PD group without is also remarkable. One would expect that this difference would be the other way around, since olfactory and sleep disturbances often go together as pre-clinical markers of $P D^{39}$. AD outcomes should however be interpreted carefully, since $A D$ changes are hard to interpret regarding the underlying tissue structure ${ }^{40}$. Misinterpretations of results can especially take place if the signal-to-noise ratio is low, if crossing fibers are present, or if pathology causes a decrease in anisotropy ${ }^{41,42}$. Results should also be interpreted carefully since it is expected that differences in $A D$ and RD values (AD being parallel to axons, and RD being perpendicular to axons) should be in the opposite direction 
${ }^{40}$, but this was not the case for our results (Table 1, Supplementary Table 1, Supplementary Table 2).

Our methods most resemble the recent study by Nigro et al., 2020, which also used OT tractography of DWI images of a recently diagnosed PD group and a HC group. PD and HC groups were comparable to the groups in our study regarding age and disease duration. The main differences between the study performed by Nigro et al., 2020 and our study is the difference in MRI field strength (3T versus $7 T$ ) and the assessment of smell capabilities. Their PD group had a decreased ability to smell and to detect odours compared to their HC group. The fact that we did not find any differences in diffusion measures of the OT between groups may be caused by our unselected group of PD patients, or by the higher field strength used in this study. Although the use of 7T DWI may result in more precise targeting of the OT, DWI is one of the techniques for which the actual gain of using $7 \mathrm{~T}$ is not obvious ${ }^{43}$, especially since the OT is anatomically close to air-filled sinuses which make this region more prone to susceptibility artifacts.

Other studies also included PD patients with significantly decreased measures of odour discrimination, identification and threshold compared to the included HC group ${ }^{14}$. Therefore, the included groups were indistinguishable along two axes; PD compared to HC and hyposmic compared to normosmic. In the current study, the ability to smell and to detect odours was not taken into account. We believe that in order to be useful as a biomarker for early PD in the general population, diffusion measures of the OT should be able to distinguish PD from $\mathrm{HC}$ regardless of the ability to smell. Also, this study used a large sample size ( $n=62$ for PD, $n=27$ for $\mathrm{HC}$ ) and only early PD patients were included (average disease duration < 19 months). In addition, extensive quality control of the diffusion data was performed and we were able to reliably trace the OT for all participants.

In conclusion, this study showed that fiber tracking of the OT was feasible in both early PD and HC using 7T DWI data. No significant differences were found in DWI measures between PD patients and HC. Results do suggest that PD patients show accelerated OT degeneration compared to $\mathrm{HC}$. However, in order to serve as a clinically relevant biomarker, the diffusion measures of the OT should differ between early PD patients and HC, without taking into account their ability to smell. We therefore conclude, based on the presented results, that 7T diffusion measures of the OT are not useful as an early clinical biomarker for PD. Future work is needed to clarify the role of other OT measurements as a biomarker for PD and its different subgroups. 


\section{Supplementary materials}
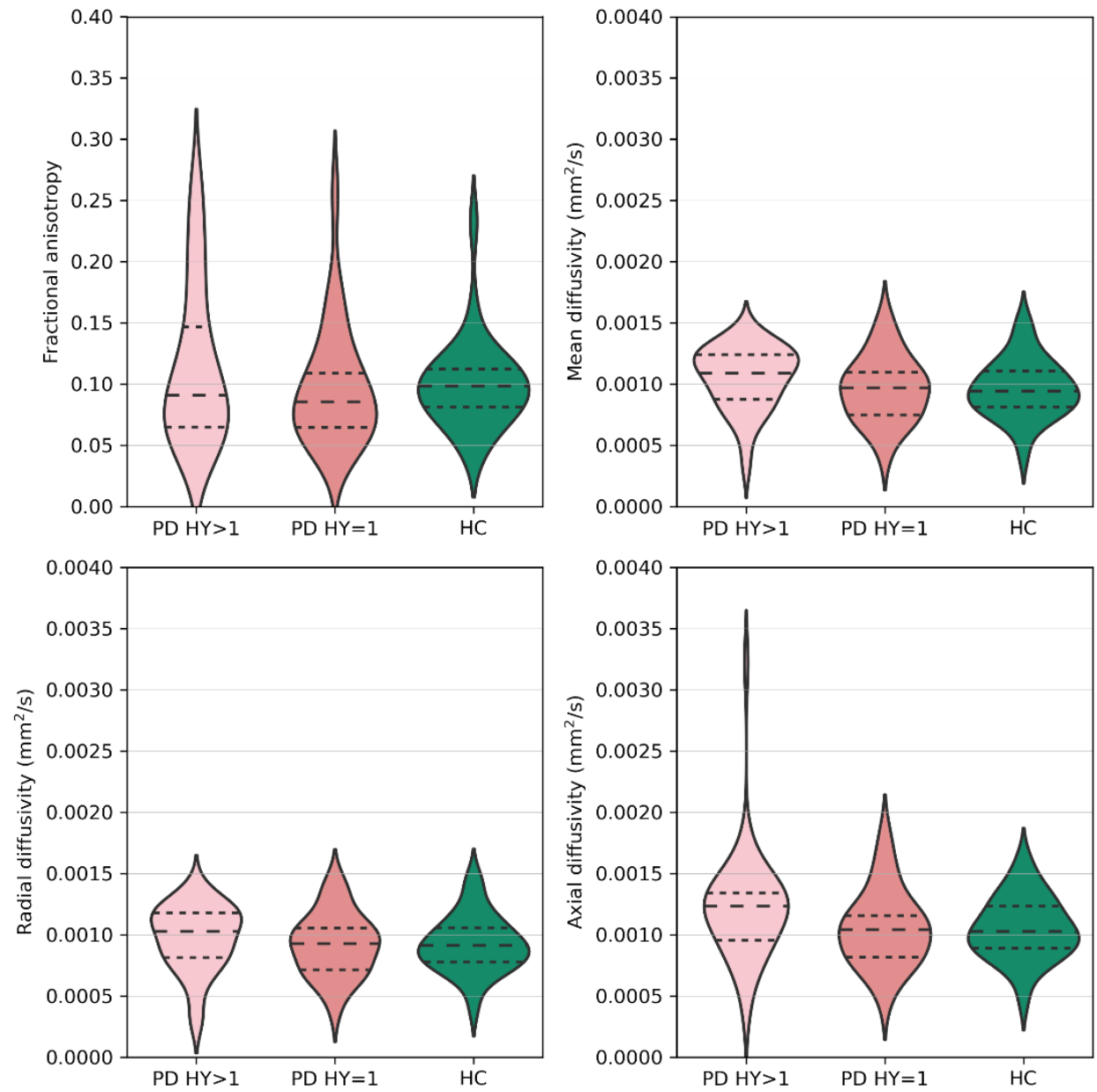

Supplementary Figure 1. Fractional anisotropy, mean diffusivity, radial diffusivity and axial diffusivity values of the olfactory tract of Parkinson's disease patients with Hoehn and Yahr scores > 1 (HY234), Parkinson's disease patients with Hoehn and Yahr scores $=1$ (HY1) and healthy controls (HC). Dashed lines represent the quartiles of the distribution. 

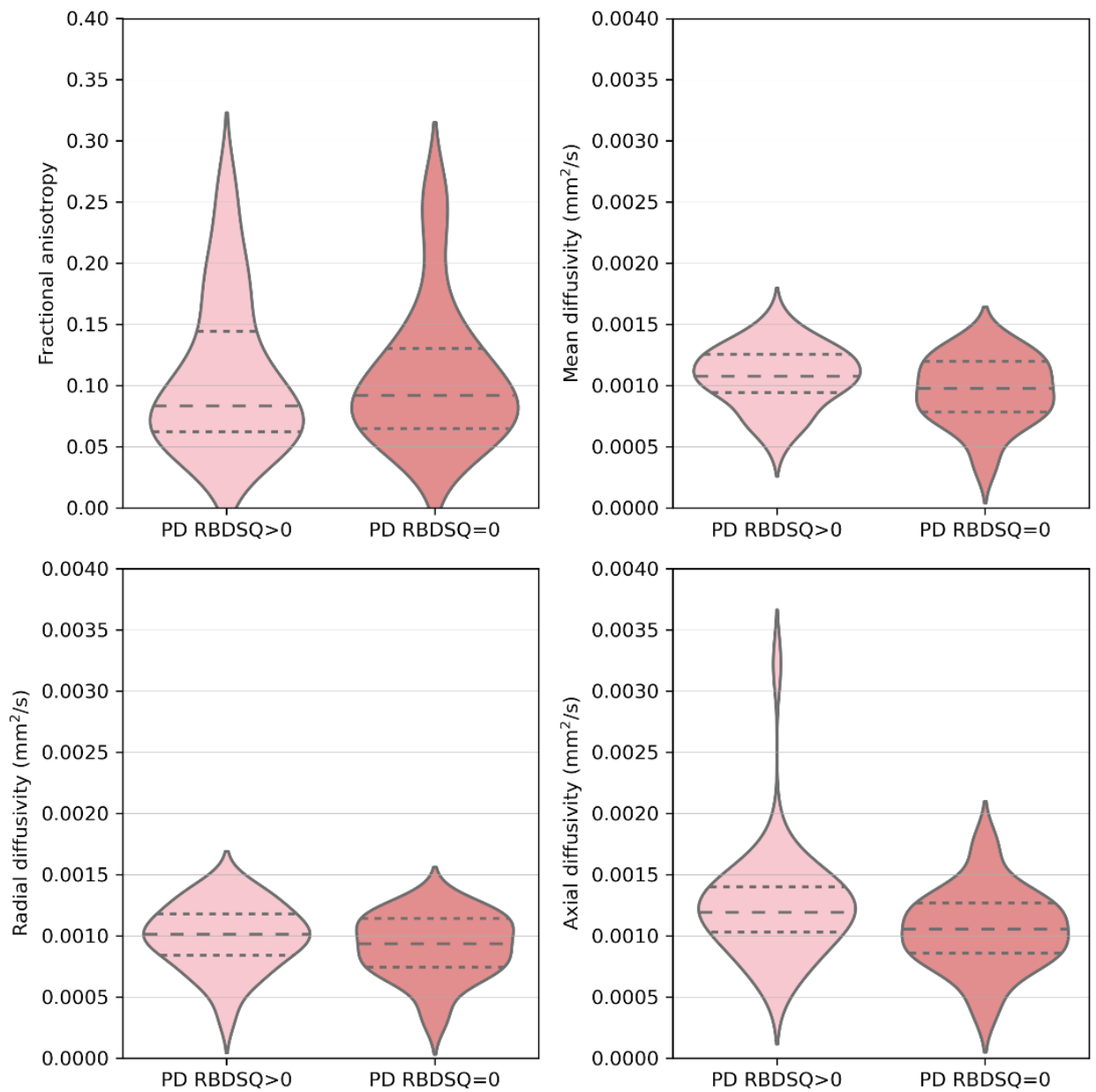

Supplementary Figure 2. Fractional anisotropy, mean diffusivity, radial diffusivity and axial diffusivity values of the olfactory tract of Parkinson's disease patients with REM sleep behaviour disorder (YES) and Parkinson's disease patients without REM sleep behaviour disorder (NO). Dashed lines represent the quartiles of the distribution. 
Supplementary Table 1. Diffusion measures of the olfactory tract of Parkinson's disease patients with Hoehn and Yahr scores > 1, Parkinson's disease patients with Hoehn and Yahr scores $=1$ and healthy controls.

\begin{tabular}{|c|c|c|c|c|}
\hline & $\begin{array}{l}\text { PD Hoehn\&Yahr }>1 \\
(n=38)\end{array}$ & $\begin{array}{l}\text { PD Hoehn\&Yahr = } 1 \\
(\mathrm{n}=24)\end{array}$ & $\begin{array}{l}\text { Healthy controls } \\
(n=27)\end{array}$ & $P$ value \\
\hline FA & $0.114946(0.064510)$ & $0.098155(0.048476)$ & $0.099815(0.036433)$ & 0.6117 \\
\hline $\mathrm{MD}\left(\mathrm{mm}^{2} / \mathrm{s}\right)$ & 0.00 & 0.00 & 6) & 15 \\
\hline $\mathrm{RD}\left(\mathrm{mm}^{2} / \mathrm{s}\right)$ & $0.000970(0.000268)$ & $0.000921(0.000255)$ & $0.000923(0.000230)$ & 0.6790 \\
\hline $\mathrm{AD}\left(\mathrm{mm}^{2} / \mathrm{s}\right)$ & $0.001217(0.000443)$ & $0.001054(0.000312)$ & $0.001051(0.000253)$ & 0.0521 \\
\hline
\end{tabular}

Data are presented as mean (std). $F A=$ fractional anisotropy, $M D=$ mean diffusivity, $R D=$ radial diffusivity, $A D=$ axial diffusivity

Supplementary Table 2. Diffusion measures of the olfactory tract of Parkinson's disease patients with and without REM sleep behaviour disorder.

\begin{tabular}{lll}
$\begin{array}{l}\text { PD with REM sleep } \\
\text { behaviour disorder RBDSQ }>\mathbf{1} \\
(\mathbf{n}=\mathbf{3 4})\end{array}$ & $\begin{array}{l}\text { PD without REM sleep } \\
\text { behaviour disorder RBDSQ }=\mathbf{0} \\
(\mathbf{n}=\mathbf{2 8})\end{array}$ & P value \\
\hline $0.108579(0.061423)$ & $0.108285(0.056982)$ & 0.7933 \\
$0.001072(0.000246)$ & $0.000955(0.000270)$ & 0.0681 \\
$0.000987(0.000266)$ & $0.000908(0.000255)$ & 0.2509 \\
$0.001241(0.000448)$ & $0.001049(0.000317)$ & 0.0377
\end{tabular}

Data are presented as mean (std). $F A=$ fractional anisotropy, $M D=$ mean diffusivity, $R D=$ radial diffusivity, $A D=$ axial diffusivity 


\section{References}

1. Postuma, R. B. et al. MDS clinical diagnostic criteria for Parkinson's disease. Mov. Disord. Off. J. Mov. Disord. Soc. 30, 1591-1601 (2015).

2. Hughes, A. J., Daniel, S. E., Ben-Shlomo, Y. \& Lees, A. J. The accuracy of diagnosis of parkinsonian syndromes in a specialist movement disorder service. Brain J. Neurol. 125, 861-870 (2002).

3. Isaias, I. U. et al. Neuromelanin Imaging and Dopaminergic Loss in Parkinson's Disease. Front. Aging Neurosci. 8, 196 (2016).

4. Lehericy, S. et al. The role of high-field magnetic resonance imaging in parkinsonian disorders: Pushing the boundaries forward. Mov. Disord. Off. J. Mov. Disord. Soc. 32, 510-525 (2017).

5. Prange, S., Metereau, E. \& Thobois, S. Structural Imaging in Parkinson's Disease: New Developments. Curr. Neurol. Neurosci. Rep. 19, 50 (2019).

6. Schwarz, S. T. et al. The 'Swallow Tail' Appearance of the Healthy Nigrosome - A New Accurate Test of Parkinson's Disease: A Case-Control and Retrospective Cross-Sectional MRI Study at 3T. PLoS ONE 9, (2014).

7. Wolters, A. F. et al. The TRACK-PD study: protocol of a longitudinal ultra-high field imaging study in Parkinson's disease. BMC Neurol. 20, (2020).

8. Basser, P. J., Mattiello, J. \& LeBihan, D. MR diffusion tensor spectroscopy and imaging. Biophys. J. 66, 259-267 (1994).

9. Alexander, A. L., Lee, J. E., Lazar, M. \& Field, A. S. Diffusion tensor imaging of the brain. Neurother. J. Am. Soc. Exp. Neurother. 4, 316-329 (2007).

10. Mori, S. \& Zhang, J. Principles of diffusion tensor imaging and its applications to basic neuroscience research. Neuron 51, 527-539 (2006).

11. Braak, H., Ghebremedhin, E., Rüb, U., Bratzke, H. \& Del Tredici, K. Stages in the development of Parkinson's disease-related pathology. Cell Tissue Res. 318, 121-134 (2004).

12. Atkinson-Clement, C., Pinto, S., Eusebio, A. \& Coulon, O. Diffusion tensor imaging in Parkinson's disease: Review and meta-analysis. Neurolmage Clin. 16, 98-110 (2017).

13. Rolheiser, T. M. et al. Diffusion tensor imaging and olfactory identification testing in early-stage Parkinson's disease. J. Neurol. 258, 1254-1260 (2011).

14. Scherfler, C. et al. Correlation of dopaminergic terminal dysfunction and microstructural abnormalities of the basal ganglia and the olfactory tract in Parkinson's disease. Brain J. Neurol. 136, 3028-3037 (2013).

15. Georgiopoulos, C. et al. Olfactory Impairment in Parkinson's Disease Studied with Diffusion Tensor and Magnetization Transfer Imaging. J. Park. Dis. 7, 301-311 (2017).

16. Nigro, P. et al. Changes of olfactory tract in Parkinson's disease: a DTI tractography study. Neuroradiology (2020) doi:10.1007/s00234-020-02551-4.

17. Stiasny-Kolster, K. et al. Combination of 'idiopathic' REM sleep behaviour disorder and olfactory dysfunction as possible indicator for $\alpha$-synucleinopathy demonstrated by dopamine transporter FPCIT-SPECT. Brain 128, 126-137 (2005).

18. Nasreddine, Z. S. et al. The Montreal Cognitive Assessment, MoCA: a brief screening tool for mild cognitive impairment. J. Am. Geriatr. Soc. 53, 695-699 (2005).

19. Goetz, C. G. et al. Movement Disorder Society-sponsored revision of the Unified Parkinson's Disease Rating Scale (MDS-UPDRS): scale presentation and clinimetric testing results. Mov. Disord. Off. J. Mov. Disord. Soc. 23, 2129-2170 (2008).

20. Goetz, C. G. et al. Movement Disorder Society Task Force report on the Hoehn and Yahr staging scale: status and recommendations. Mov. Disord. Off. J. Mov. Disord. Soc. 19, 1020-1028 (2004).

21. Visser, M., Marinus, J., Stiggelbout, A. M. \& Van Hilten, J. J. Assessment of autonomic dysfunction in Parkinson's disease: the SCOPA-AUT. Mov. Disord. Off. J. Mov. Disord. Soc. 19, 1306-1312 (2004).

22. Stiasny-Kolster, K. et al. The REM sleep behavior disorder screening questionnaire--a new diagnostic instrument. Mov. Disord. Off. J. Mov. Disord. Soc. 22, 2386-2393 (2007).

23. Teeuwisse, W. M., Brink, W. M. \& Webb, A. G. Quantitative assessment of the effects of highpermittivity pads in 7 Tesla MRI of the brain. Magn. Reson. Med. 67, 1285-1293 (2012). 
24. Griswold, M. A. et al. Generalized autocalibrating partially parallel acquisitions (GRAPPA). Magn. Reson. Med. 47, 1202-1210 (2002).

25. Li, X., Morgan, P. S., Ashburner, J., Smith, J. \& Rorden, C. The first step for neuroimaging data analysis: DICOM to NIfTI conversion. J. Neurosci. Methods 264, 47-56 (2016).

26. Kellner, E., Dhital, B., Kiselev, V. G. \& Reisert, M. Gibbs-ringing artifact removal based on local subvoxel-shifts. Magn. Reson. Med. 76, 1574-1581 (2016).

27. Veraart, J., Fieremans, E. \& Novikov, D. S. Diffusion MRI noise mapping using random matrix theory. Magn. Reson. Med. 76, 1582-1593 (2016).

28. Veraart, J. et al. Denoising of diffusion MRI using random matrix theory. Neurolmage 142, 394-406 (2016).

29. Andersson, J. L. R., Skare, S. \& Ashburner, J. How to correct susceptibility distortions in spin-echo echo-planar images: application to diffusion tensor imaging. 19 (2003).

30. Andersson, J. L. R. \& Sotiropoulos, S. N. An integrated approach to correction for off-resonance effects and subject movement in diffusion MR imaging. Neurolmage 125, 1063-1078 (2016).

31. Smith et al. Advances in functional and structural MR image analysis and implementation as FSL. Neuroimage 23 Suppl 1, S208-19 (2004).

32. Bastiani, M. et al. Automated quality control for within and between studies diffusion MRI data using a non-parametric framework for movement and distortion correction. Neurolmage 184, 801-812 (2019).

33. Tournier, J.-D., Calamante, F. \& Connelly, A. Determination of the appropriate b value and number of gradient directions for high-angular-resolution diffusion-weighted imaging. NMR Biomed. 26, 17751786 (2013).

34. Tournier, J.-D., Calamante, F. \& Connelly, A. Robust determination of the fibre orientation distribution in diffusion MRI: non-negativity constrained super-resolved spherical deconvolution. Neurolmage 35, 1459-1472 (2007).

35. Tournier, J.-D., Calamante, F. \& Connelly, A. MRtrix: Diffusion tractography in crossing fiber regions. Int. J. Imaging Syst. Technol. 22, 53-66 (2012).

36. Basser, P. J., Mattiello, J. \& LeBihan, D. Estimation of the effective self-diffusion tensor from the NMR spin echo. J. Magn. Reson. B 103, 247-254 (1994).

37. Bartko, J. J. The Intraclass Correlation Coefficient as a Measure of Reliability. Psychol. Rep. 19, 3-11 (1966).

38. Stebbins, G. T. \& Murphy, C. M. Diffusion tensor imaging in Alzheimer's disease and mild cognitive impairment. Behav. Neurol. 21, 39-49 (2009).

39. Miller, D. B. \& O'Callaghan, J. P. Biomarkers of Parkinson's disease: present and future. Metabolism. 64, S40-46 (2015).

40. Wheeler-Kingshott, C. A. M. \& Cercignani, M. About "axial" and "radial" diffusivities. Magn. Reson. Med. 61, 1255-1260 (2009).

41. Wheeler-Kingshott, C. A. M., Ciccarelli, O., Schneider, T., Alexander, D. C. \& Cercignani, M. A new approach to structural integrity assessment based on axial and radial diffusivities. Funct. Neurol. 27, 85-90 (2012)

42. Winklewski, P. J. et al. Understanding the Physiopathology Behind Axial and Radial Diffusivity Changes-What Do We Know? Front. Neurol. 9, (2018).

43. Polders, D. L. et al. Signal to noise ratio and uncertainty in diffusion tensor imaging at 1.5, 3.0, and 7.0 Tesla. J. Magn. Reson. Imaging 33, 1456-1463 (2011). 


\section{Chapter 7}

\section{Variability in subthalamic nucleus targeting}

for deep brain stimulation with 3 and 7 Tesla magnetic resonance imaging

B. R. Isaacs*, M. Heijmans*, M.L. Kuijf, P.L. Kubben, L. Ackermans, Y. Temel, M.C. Keuken,

B. U. Forstmann

*shared first authors

Revised version published. Neuroimage: Clinical 2021; 32 


\begin{abstract}
Deep brain stimulation (DBS) of the subthalamic nucleus (STN) is an effective surgical treatment for Parkinson's disease (PD). Side-effects may, however, be induced when the DBS lead is placed suboptimally. Currently, lower field magnetic resonance imaging (MRI) at 1.5 or 3 Tesla (T) is used for targeting. Ultra-high-field MRI (7T and above) can obtain superior anatomical information and might therefore be better suited for targeting. This study aims to test whether optimized 7T imaging protocols result in less variable targeting of the STN for DBS compared to clinically utilized 3T images. Three DBS-experienced neurosurgeons determined the optimal STN DBS target site on three repetitions of 3T-T2, 7T-T2* 7T-R2* and 7T-QSM images for five PD patients. The distance in millimetres between the three repetitive coordinates was used as an index of targeting variability and was compared between field strength, MRI contrast and repetition with a Bayesian ANOVA. Further, the target coordinates were registered to $\mathrm{MNI}$ space, and anatomical coordinates were compared between field strength, MRI contrast and repetition using a Bayesian ANOVA. The results indicate that the neurosurgeons are stable in selecting the DBS target site across MRI field strength, MRI contrast and repetitions. The analysis of the coordinates in $\mathrm{MNI}$ space however revealed that the actual selected location of the electrode is seemingly more ventral when using the 3T scan compared to the 7T scans.
\end{abstract}

\title{
Keywords
}

Deep brain stimulation; field strength; magnetic resonance imaging; subthalamic nucleus; targeting 


\section{Introduction}

Since its introduction in the 1990s, deep brain stimulation (DBS) of the subthalamic nucleus (STN) has proven to be an effective surgical treatment for advanced Parkinson's disease (PD) ${ }^{1,2}$. STN DBS for PD is especially efficacious in treating otherwise refractory tremor, motor fluctuations and dyskinesias ${ }^{3,4}$. However, in spite of these positive outcomes, STN DBS has the potential to induce a number of side-effects including behavioral changes, cognitive impairments and speech, balance or gait problems ${ }^{5-8}$. These side-effects may be a product of suboptimal placement of the DBS lead ${ }^{9-11}$. Here we focus on the first of many procedural steps that can contribute to such suboptimal placement; stereotactic planning of the electrode site ${ }^{12}$.

Targeting the STN can either be done using a constant coordinate relative to a given anatomical landmark or by visualizing the STN per individual and determining the target per patient. These two approaches are respectively called indirect and direct targeting, where direct targeting typically results in a better patient outcome as individual anatomical variability is taken into account ${ }^{13}$. Common clinical practices for direct targeting of the STN for DBS is achieved using T2-weighted (-w) magnetic resonance imaging (MRI) ${ }^{14,15}$. T2-W MRI is sensitive to iron content, and the STN is rich in iron, which causes it to appear hypointense compared to the surrounding grey matter structures ${ }^{16,17}$. Additionally or alternatively, some centers incorporate intraoperative microelectrode recordings (MER) for target verification, while others rely exclusively on indirect targeting approaches with MER and standardized coordinate systems ${ }^{18}$. Notably, most centers perform the DBS surgery in awake settings with local anaesthesia, thereby enabling the clinician to assess stimulation related side-effects during test stimulation and adjustment of the final electrode targeting. STN surgeries are increasingly being performed under general anaesthesia and therefore the precision of the stereotactic planning with MRI is becoming even more important.

Direct targeting for DBS traditionally relies on lower field MRI (1.5 and 3T MRI) which are prone to low contrast and signal to noise ratios (CNR and SNR, respectively), and result in images that lack sharp and clear borders of small deep brain structures ${ }^{19,20}$. Ultra-high-field MRI systems (7T and above) can obtain submillimeter anatomical information with increased contrast ${ }^{21,22}$. Whether the benefits of ultra-high-field MRI result in better targeting for DBS remains unclear ${ }^{23-28}$.

In addition to higher field strengths, quantitative imaging methods may contribute to the visualisation of DBS targets as they convey microstructural properties of the area of interest. For example, while T2* contrasts visualize the STN as a hypointense structure, they can provide additional quantitative maps that provide information in relation to iron content and load ${ }^{29-31}$. Further, effective transverse relaxation rate, or $\mathrm{R} 2{ }^{*}$, maps 
$(\mathrm{R} 2 *=1 / \mathrm{T} 2 *)$ derived from $\mathrm{T} 2 *$ contrasts are even more sensitive to iron load and visualize the STN as a hyperintense structure ${ }^{32}$. T2* contrasts can be processed into Quantitative Susceptibility Maps (QSM) which are also sensitive to iron. However, contrary to $2^{*}$ and R2* based modalities, QSM accounts for local susceptibility inhomogeneities by incorporating both magnitude and phase image information as well as incorporating methods to remove background fields such as a dipole convolution ${ }^{33,34}$. This has led some groups to suggest that QSM is the superior contrast for imaging subcortical structures that are high in iron content ${ }^{27,35}$.

Once the STN is visualized the question still remains where to place the electrode. While the exact optimal site of stimulation within the STN varies per patient ${ }^{36,37}$, the general consensus is that DBS is most effective in treating PD when the lead is placed in the dorsolateral (sensorimotor) portion of the nucleus ${ }^{25,38-40}$. When targeting towards the ventral (limbic) portion of the STN, cognitive and psychiatric side-effects are more likely to occur ${ }^{41}$. Or in the words of lead-DBS core-developer Andreas Horn 'Millimetres matter' when it comes to DBS ${ }^{42}$. The precision of electrode target selection is therefore considered to be one of the first of many important factors that determine DBS outcome but the reproducibility, to the best of our knowledge, has not been formally investigated.

This study aims to test whether optimized $7 \mathrm{~T}$ imaging protocols including $\mathrm{T} 2 *, \mathrm{R} 2 *$ and QSM contrasts result in less variable targeting for STN DBS than clinically utilized 3T T2 scans. Three neurosurgeons targeted, what they considered the optimal STN DBS site, on 3 repetitions of $3 \mathrm{~T}-\mathrm{T} 2,7 \mathrm{~T}-\mathrm{T} 2 *$, 7T-R2* and 7T-QSM images for five PD patients ${ }^{1}$. A low degree of variability across repetitions would indicate that the MR image allows for a consensus view as to the optimal target location, whereas a high degree of variability would indicate that the image lacks the required visibility to reach a unanimous agreement. We do not focus on the performance of the individual neurosurgeons, but we specifically focus on the amount of variability in the targeted coordinates of the various MR image modalities. We hypothesize that the test-retest reliability of STN targeting will be higher for the optimized 7T contrasts than for the clinically utilized 3T images. Further, we hypothesize that the test-retest reliability of STN targeting on 7T-QSM contrasts will be higher compared to $7 \mathrm{~T}-\mathrm{T} 2 *$ or R2* as previous literature has suggested that QSM is superior in imaging the STN at 7T. A second aim is to test whether different MRI contrasts can result in different target locations as each MRI contrast contains complimentary anatomical information ${ }^{43-45}$.

\section{Materials and Methods}

\section{Participants}

A total of five PD patients participated in the study $(M=4 ; F=1)$ with a mean age of 62.2 years ( $S D=7.9$ years) and a mean number of 8.4 years since the official diagnoses $(S D=3.6$ 108 
years). PD patients were recruited as candidates for DBS surgery at the Neurology department within the Maastricht University Medical Centre (The Netherlands). The study was approved by the local Medical Ethical Committee at the Maastricht University Medical Centre (NL60342.068.17/METC172010). All data was collected and is held in accordance with the EU General Data Protection Regulation (GDPR) and the Dutch Act on Implementation of the GDPR, good clinical practice and relevant data protection laws. PD patients had no diagnosed neurological comorbidities and provided written informed consent prior to the scanning.

\section{Data acquisition}

\section{Tesla MRI}

Each PD patient underwent a preoperative clinical 3T scan as part of the standard clinical practice with a Phillips Ingenia scanner using a 32-channel head coil at the Maastricht University Medical Center. The 3T data that was obtained consisted of the standard clinical sequences used for DBS planning at the Maastricht University Medical Center. A whole brain 3D turbo field echo (TFE) T1-w scan was obtained with $1 \mathrm{~mm}$ isotropic voxel sizes, with the following parameters: Repetition Time $(T R)=8.1 \mathrm{~ms}$, Echo Time $(T E)=3.7 \mathrm{~ms}$, Inversion Recovery $(\mathrm{IR})$ delay $=776 \mathrm{~ms}$, Flip Angle $(\mathrm{FA})=8^{\circ}$, Bandwidths $(\mathrm{BW})=191.5 \mathrm{~Hz} / \mathrm{px}$, Echo Spacing $(E S)=13.6 \mathrm{~ms}$, TFE factor $=183$, transverse orientation acquisition in the anterior-posterior direction, with SENSE factor of 1.4 and total acquisition time (TA) of 05:51mins. A whole brain T2-w scan was obtained with spin echo sequence with $0.45 \times 0.45$ $x 2 \mathrm{~mm}$ voxel sizes, with the following parameters: 65 slices, $\mathrm{TR}=8264 \mathrm{~ms}$, TE $80 \mathrm{~ms}, \mathrm{FA}=$ $90^{\circ}, \mathrm{BW}=193.6 \mathrm{~Hz} / \mathrm{px}$, TFE factor $=15$, transverse orientation acquisition in the anteriorposterior direction, with SENSE factor of 1.5 and TA of $06: 20 \mathrm{mins}$.

\section{Tesla MRI}

In addition to the standard clinical 3T acquisition, a 7T scan was acquired with a Siemens Magnetom scanner using a 32-channel head coil at the Scannexus Centre for Neuroimaging in Maastricht. Whole brain T1-w 3D images were obtained with an adapted version of the multi echo MP2RAGE (magnetization-prepared rapid gradient echo multi-echo) sequence 46,47 with $0.8 \mathrm{~mm}$ isotropic voxel sizes and the following parameters: 208 slices, $\mathrm{TR}=$ $6000 \mathrm{~ms}, \mathrm{TE}_{1,2}=[2.74 \mathrm{~ms}, 8.71 \mathrm{~ms}]$, Inversion Time $(\mathrm{TI})_{1,2}=[750 \mathrm{~ms}, 29000 \mathrm{~ms}], \mathrm{FA}_{1,2}=\left[4^{\circ}\right.$, $\left.6^{\circ}\right], \mathrm{BW}_{1,2}=[350 \mathrm{~Hz} / \mathrm{Px}, 150 \mathrm{~Hz} / \mathrm{Px}], \mathrm{ES}=13.6 \mathrm{~ms}$, interleaved and single shot multi slice mode and interleaved, sagittal orientation acquisition in the anterior-posterior direction, phase partial Fourier 6/8, parallel acquisition with GRAPPA and acceleration factor of 3 and TA of 10:56mins. Where possible, dielectric pads were placed between the side of the participants head and the receiver coil to reduce $B_{1}$ inhomogeneity artefacts. The $T 2 *-W$ 3D scan was acquired with a partial volume GRE (gradient echo) ASPIRE (multi-channel phase data from multi-echo acquisitions) sequence covering the subcortex with $0.5 \mathrm{~mm}$ 
isotropic voxel sizes and the following parameters: 90 slices, $16.7 \%$ slice oversampling, TR $=33 \mathrm{~ms}, \mathrm{TE}_{1-4}=[2.49 \mathrm{~ms}, 6.75 \mathrm{~ms}, 13.50 \mathrm{~ms}, 20.75 \mathrm{~ms}], \mathrm{FA}=12^{\circ}, \mathrm{BW}{ }_{1-4}=[300 \mathrm{~Hz} / \mathrm{px}$, $300 \mathrm{~Hz} / \mathrm{px}, 200 \mathrm{~Hz} / \mathrm{px}, 100 \mathrm{~Hz} / \mathrm{px}$ ], interleaved multi slice mode, sagittal orientation acquisition in the anterior-posterior direction, slice partial Fourier $7 / 8$, parallel acquisition with GRAPPA and acceleration factor of 2 and TA 07.42 mins ${ }^{48}$.

\section{Calculation of Quantitative MRI Maps}

All quantitative maps were created in native space. First, skull information was removed using the Brain Extraction Tool as implemented in FSL 5.0 ${ }^{49,50}$. The 3T T2-w MRI sequence did not allow the calculation of quantitative maps due to the acquisition parameters. The maps for 7T MRI scans were created using the following procedure: T2*-maps were computed by least-squares fitting of the exponential signal decay over the four echoes of magnitude image from the GRE ASPIRE sequence ${ }^{51}$. R2* maps were then calculated by taking the reciprocal of the T2* map. For QSM, phase maps of the fourth echo were preprocessed using iHARPERELLA (integrated phase unwrapping and background phase removal using the Laplacian) and used to calculate QSM with LSQR (sparse linear equation and least-squares method) ${ }^{52-54}$.

\section{Targeting the STN}

Identification of the STN was conducted by a total of three neurosurgeons with a mean experience of 13.7 years (SD = 5.7 years) in STN DBS planning and surgery. Each neurosurgeon targeted separate left and right STNs per participant on the following image modalities: 3T-T2, 7T-T2*, 7T-R2* and 7T-QSM. All scans used to target the STN were in native acquisition space. The targeting procedure was repeated three times for every image and was assigned a novel identifier, so the neurosurgeons were unaware of the identification of each patient and repetition. The targeting procedure of the STN is shown in Figure 1. Order of presentation of the images was fixed and the same for all three neurosurgeons. There were no images of the same participant following each other. Images were automatically loaded and presented in FSLeyes with pre-set intensity levels using an in-house Bash script. The masks were marked with the anonymized patient identifier, hemisphere and initial of the targeting neurosurgeon. The neurosurgeons then identified the coordinate in which they would place the DBS electrode, and a screenshot of this coordinate was saved. A total of 120 STN targets were obtained per neurosurgeon, and targeting was achieved in multiple blocks over a period of approximately 3 months.

As the neurosurgeons were more used to planning on $3 \mathrm{~T}$, instructions and examples were provided to explain the $7 \mathrm{~T}$ images with the following: $\mathrm{i}$. 'T2* images provide an indirect measure of iron content. Iron rich regions like the STN show a higher magnetic field perturbation compared to adjacent regions with lower iron content. The STN appears as a hypointense structure'. li. 'R2* maps offer a direct measure of magnetism. The STN appears 110 
as a hyperintense structure'. lii. 'QSM (quantitative susceptibility maps) are post processed images based on the fourth echo of the $\mathrm{T} 2^{*}$ sequence, and invert the image contrast, also allowing for a direct measure of magnetism per voxel. The STN appears as a hyperintense structure'. The neurosurgeons were asked to define the position where they would place the electrode tip without taking the corresponding trajectory into account. An example of the intended electrode tip location for a patient by a single neurosurgeon is given in Figure 2.

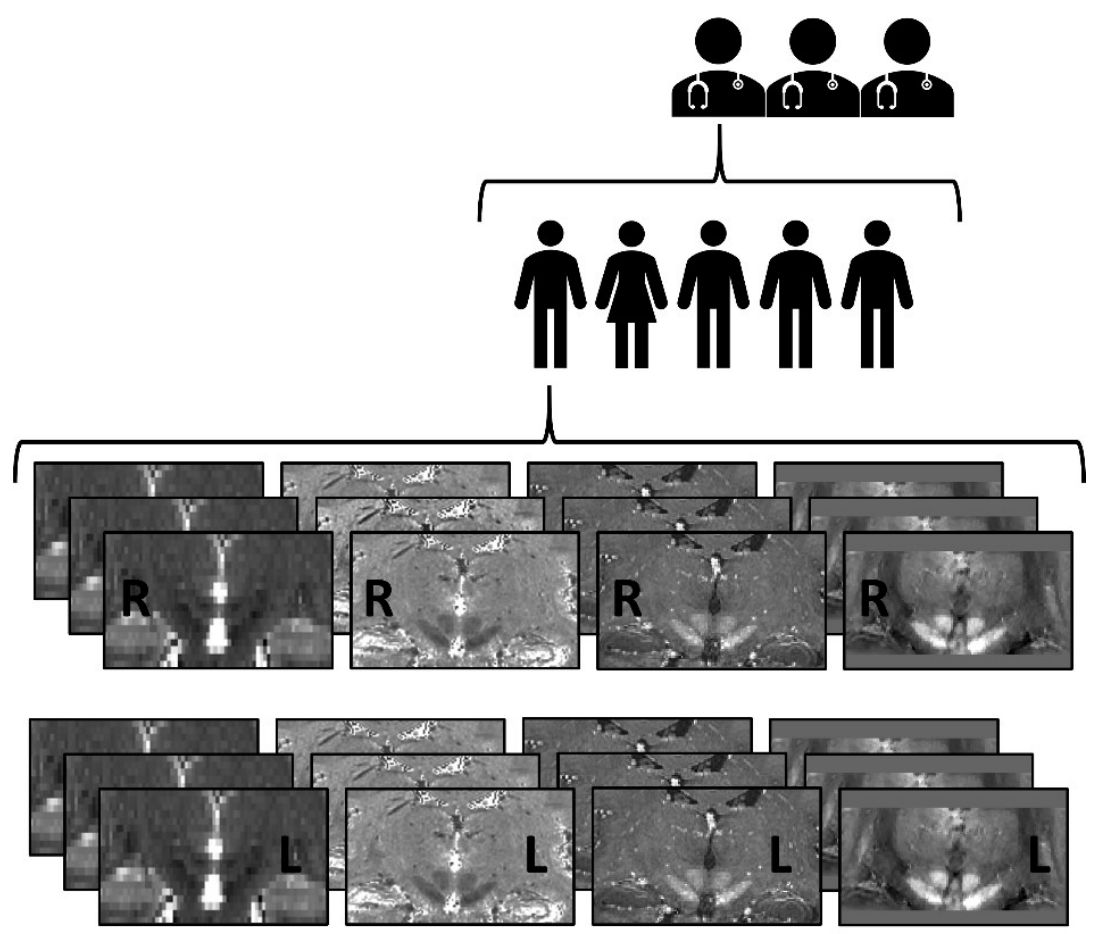

Figure 1. Targeting procedure of the subthalamic nucleus (STN). Identification was conducted by three neurosurgeons. Each neurosurgeon targeted the STN for five deep brain stimulation (DBS) candidates suffering from Parkinson's Disease (PD), on four image modalities, with three repetitions, for both left and right STN. The image modalities included (from left to right) a 3T-T2 weighted MRI, 7T-T2* map, 7T-R2* map and 7T Quantitative Susceptibility Map (QSM). This resulted in a total of 120 STN targets per neurosurgeon, with 24 targets per patient.

\section{Euclidean Distance}

The Euclidean distance (from here onwards called distance) between the repetitive coordinates was used as an index of variability, where smaller distances indicate better test-retest reliability ${ }^{55}$. The distance was calculated between the first and second repetition, second and third repetition, and first and third repetition. This resulted in three 
distance pairs per hemisphere for each contrast, subject, and neurosurgeon, or 90 distance pairs in total per MRI contrast. Due to technical errors six target coordinates were not saved correctly and are therefore missing from the dataset (resp. two 3T coordinates and four 7T coordinates), resulting in 348 distance pairs in total. To account for differences in voxel geometry between the $3 \mathrm{~T}$ and $7 \mathrm{~T}$ contrasts, the voxel coordinate of the target was transformed to millimetres by multiplying the $x$ and $y$ voxel coordinate values with, respectively, 0.44921875 or 0.53125 for the $3 T$ and $7 T$ coordinates and the $z$ voxel coordinate values with, respectively, 2.0 or 0.5300006 . This ensured that a direct comparison between the $3 \mathrm{~T}$ and $7 \mathrm{~T}$ derived distances was possible.

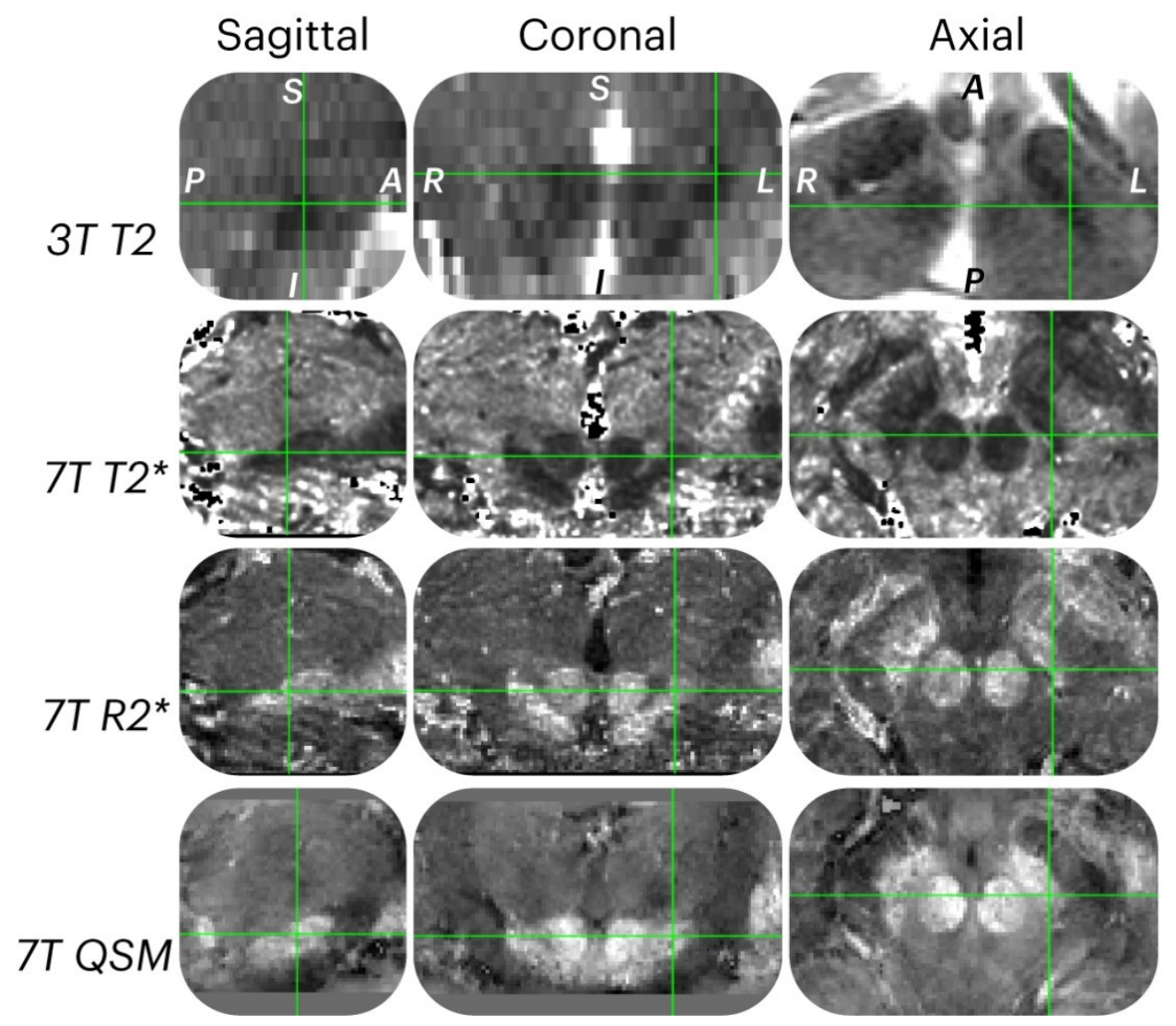

Figure 2. MRI image modalities. An example of each MRI image modality used for targeting for a single patient, including from top to bottom, a 3T-T2 weighted MRI, 7T-T2* map, 7T-R2* map and 7T Quantitative Susceptibility Map (QSM). The image shows a zoomed section of the subthalamic nucleus (STN) in the sagittal, coronal and axial planes. The STN is highlighted by the green intersection. 


\section{Target coordinates in standard stereotactic MNI-space}

To be able to compare the location of the target coordinate across subjects and MRI contrasts it was necessary to estimate the $3 \mathrm{~T}$ and $7 \mathrm{~T}$ slab transformations to standard MNI space. All individual scans were skull stripped using BET as implemented in FSL 5.0. The 3T $\mathrm{T} 2-\mathrm{w}$ and the average of the four $7 \mathrm{~T} \mathrm{~T} 2 *$ volumes were registered to the 7T whole brain T1w scan using a rigid transformation 'DenseRigid' as implemented in ANTsPy. The 7T whole brain T1-w scan was registered to the icbm_avg_152_t1_tal_nlin_symmetric_VI 1mm isotropic MNI template using the Symmetric normalization as implement in ANTsPy. This is a combination of affine and deformable transformations using mutual information as the optimization metric. All registration steps were visually inspected and were considered to be reasonable. Using fslmaths a NifTi file was created for every single target coordinate in native space. The different transformation matrices were then combined with the deformation field and applied to the respective target coordinates using a bSpline interpolation. Finally, the X, Y and Z MNI coordinates of the Center of Gravity (COG) were extracted for every single target coordinate and used for further analyses. To reduce the number of statistical tests, a principal component analysis (PCA) was computed on the resulting $X, Y$, and $Z$ COG coordinates and in line with our previous work ${ }^{56}$. As we had no a-priori hypothesis regarding effects of lateralization and targeting precision, the negative $X$ coordinates (corresponding to the left hemisphere) were converted to positive values before the PCA was calculated. The resulting first principal component corresponds to a new latent variable which captures the maximal amount of variance in the $X, Y$, and $Z$ coordinates across the different target locations.

\section{Manual parcellation of the STN}

The STN was manually parcellated by two independent anatomical experts (BRI and MCK) and verified by a third independent rater $(\mathrm{MH})$, per patient, for both 3T and 7T images. The left and right hemispheres were parcellated separately. The 7T parcellations were achieved by overlaying the 7T-T2*, 7T-R2* and 7T-QSM contrasts together, to create a single 7T parcellation based on the three image modalities. Parcellations were achieved in native space and were created to assess whether any differences in test-retest or MNI location could be explained by differences in STN visibility. This was quantified by calculating the Dice coefficient. The conjunct mask only includes the voxels in the STN that were included by both raters 57 .

\section{Data analysis}

\section{Statistical methods}

All statistical analyses were conducted using ANOVAs within a Bayesian framework using the JASP software package $\left(\mathrm{V} \cdot 0.14 .1 ;{ }^{58}\right)$. The ANOVAs used a uniform prior model 
probability, and the assumption of normality were visualized using a Q-Q plot of the residuals. For both the test-retest reliability and the spatial location analyses patient ID and neurosurgeon ID were included as nuisance variables. For the Dice coefficient and volumetric analysis, the patient ID was included as a nuisance variable. The implementation of the Bayesian ANOVA in JASP relies on the R package BayesFactor (V.0.9.10-2; $\left.{ }^{59,60}\right)$. The resulting Bayes Factors (BF) are interpreted in light of assumptions proposed by ${ }^{61}$ and adapted by ${ }^{62}$. Note that the analyses regarding the test-retest reliability, Dice coefficient and volume are based on values calculated in native space whereas the spatial location analysis is based on values in MNI space.

\section{Outlier analysis}

Outliers were identified with the $1.5 x \mathrm{IQR}$ rule whereby any data point $1.5 *$ IQR above the third quartile or below the first quartile was rejected from further analysis and was done per MRI contrast or field strength. For the distance pairs, 14 data points were identified as outliers across the MRI contrasts. The final sample for the test-retest ANOVA was 84 pairs for the 3T-T2 contrast, 77 pairs for the 7T-T2* contrast, 86 pairs for the 7T-R2* contrast and 87 pairs for the 7T-QSM contrast. For the coordinates in MNI space, there were two 7T-T2* coordinates that were identified as outliers. There was a single 3T Dice coefficient value and a single $7 \mathrm{~T}$ conjunction volume that were identified as outliers.

\section{Open science}

All target coordinates and STN parcellation masks are made available (DOI 10.17605/OSF.IO/DW2FR). In addition, an annotated Python notebook that was used to pre-process all the data and all resulting JASP files used to conduct the statistical analysis are provided.

\section{Results}

\section{Test-retest reliability of the target coordinates}

On average the neurosurgeons deviated $1.35 \mathrm{~mm}(\mathrm{SD}=0.78)$ between sessions. In Table 1 the mean distances between the three targeting sessions are provided per MRI field strength and contrast whereas in Figure 3 the distance between the pairs are visualized per hemisphere and MRI contrast.

\section{Model comparison}

To test whether MRI field strength, MRI contrast or planning session had an influence on the distance between target locations a Bayesian ANOVA was conducted. The primary output from the ANOVA is presented in Table 2, which shows the amount of support that the data offer for each model under consideration. The left-most column lists all models at 
hand: 18 alternative models and a single null model. The models are ordered by their predictive performance relative to the best model; this is indicated in the $\mathrm{BF}_{10}$ column, which shows the Bayes factor relative to the best model which, in this case is the Null model. For example, the data are 5.88 times more likely under the Null model than under the second-best model where MRI field strength (Tesla) is included as a predictor. This means that there is substantial evidence that there is no effect of field strength, MRI contrast or planning session on the test-retest reliability of the STN targeting.

Table 1. Targeting distance between the repetition pairs over MRI field strengths and contrasts.

\begin{tabular}{|c|c|c|c|c|c|c|c|}
\hline \multirow[b]{2}{*}{ MRI field strength } & \multirow[b]{2}{*}{ MRI Contrast } & \multirow[b]{2}{*}{ Repetition pairs } & \multirow[b]{2}{*}{ Mean (mm) } & \multirow[b]{2}{*}{$\mathrm{SD}(\mathrm{mm})$} & \multirow[b]{2}{*}{$\mathbf{N}$} & \multicolumn{2}{|c|}{ 95\% Credible Interval } \\
\hline & & & & & & Lower & Upper \\
\hline \multirow[t]{3}{*}{$3 T$} & $\mathrm{~T} 2$ & $1-2$ & 1.197 & 0.780 & 28 & 0.895 & 1.499 \\
\hline & & $1-3$ & 1.554 & 0.934 & 29 & 1.198 & 1.909 \\
\hline & & $2-3$ & 1.433 & 0.940 & 27 & 1.061 & 1.804 \\
\hline \multirow[t]{9}{*}{$7 \mathrm{~T}$} & $\mathrm{~T} 2 *$ & $1-2$ & 1.560 & 0.902 & 26 & 1.196 & 1.924 \\
\hline & & $1-3$ & 1.178 & 0.661 & 24 & 0.899 & 1.457 \\
\hline & & $2-3$ & 1.339 & 0.814 & 27 & 1.017 & 1.661 \\
\hline & $\mathrm{R} 2 *$ & $1-2$ & 1.170 & 0.715 & 28 & 0.893 & 1.447 \\
\hline & & $1-3$ & 1.608 & 0.605 & 28 & 1.373 & 1.842 \\
\hline & & $2-3$ & 1.370 & 0.623 & 30 & 1.138 & 1.603 \\
\hline & QSM & $1-2$ & 1.266 & 0.987 & 30 & 0.898 & 1.635 \\
\hline & & $1-3$ & 1.061 & 0.623 & 28 & 0.819 & 1.302 \\
\hline & & $2-3$ & 1.467 & 0.734 & 29 & 1.188 & 1.746 \\
\hline
\end{tabular}

Note. The mean distance between sessions in millimetres and calculated over surgeon, patient and hemisphere.

\section{Spatial location of targets in MNI space}

While we can conclude that the neurosurgeons are stable in selecting the electrode target over planning sessions it is unknown whether the neurosurgeons select similar targets across MRI field strengths and MRI contrasts. For that the individual electrode target locations were registered to MNI space and visualized in Figure 4.

\section{Model comparison}

To test whether MRI field strength, MRI contrast or session had an influence on the DBS electrode location in MNI space a Bayesian ANOVA was conducted. The primary output from the ANOVA is presented in Table 3, which shows the amount of support that the data offer for each model under consideration. There is anecdotal evidence that the data is 1.74 times more likely under the model where MRI field strength is included than under the second-best model where MRI field strength and MRI contrast are included. There is however conclusive evidence that the data is more likely under the model including MRI field strength than under the Null model. 
Table 2. Model Comparison test-retest reliability of the target coordinates.

\begin{tabular}{|c|c|c|c|c|c|}
\hline Models & $\mathrm{P}(\mathrm{M})$ & $\mathrm{P}(\mathrm{M} \mid$ data $)$ & $\mathrm{BF}_{\mathrm{M}}$ & $\mathrm{BF}_{10}$ & error \% \\
\hline Null model (incl. PatientNr, Surgeon) & 0.053 & 0.781 & 64.011 & 1.000 & \\
\hline $\mathrm{T}$ & 0.053 & 0.133 & 2.751 & 0.170 & 4.842 \\
\hline $\mathrm{RP}$ & 0.053 & 0.046 & 0.876 & 0.059 & 3.877 \\
\hline $\mathrm{C}$ & 0.053 & 0.019 & 0.355 & 0.025 & 3.393 \\
\hline$T+R P$ & 0.053 & 0.007 & 0.133 & 0.009 & 1.875 \\
\hline $\mathrm{T}+\mathrm{C}$ & 0.053 & 0.007 & 0.124 & 0.009 & 2.249 \\
\hline $\mathrm{T}+\mathrm{C}+\mathrm{T} * \mathrm{~K} \mathrm{C}$ & 0.053 & 0.003 & 0.046 & 0.003 & 7.390 \\
\hline$T+R P+T * R P$ & 0.053 & 0.002 & 0.029 & 0.002 & 4.812 \\
\hline$C+R P$ & 0.053 & 0.001 & 0.019 & 0.001 & 2.113 \\
\hline$C+R P+C * R P$ & 0.053 & $6.776 e-4$ & 0.012 & $8.681 e-4$ & 1.771 \\
\hline$T+C+R P$ & 0.053 & $3.902 e-4$ & 0.007 & $5.000 e-4$ & 2.594 \\
\hline$T+C+R P+C * R P$ & 0.053 & $2.620 \mathrm{e}-4$ & 0.005 & $3.357 e-4$ & 3.145 \\
\hline $\mathrm{T}+\mathrm{C}+\mathrm{RP}+\mathrm{T} * \mathrm{~K} \mathrm{C}$ & 0.053 & $1.329 \mathrm{e}-4$ & 0.002 & $1.703 e-4$ & 2.846 \\
\hline $\mathrm{T}+\mathrm{C}+\mathrm{RP}+\mathrm{T} * \mathrm{C}+\mathrm{C} * \mathrm{RP}$ & 0.053 & $9.083 e-5$ & 0.002 & $1.164 \mathrm{e}-4$ & 2.988 \\
\hline$T+C+R P+T * R P$ & 0.053 & $7.860 e-5$ & 0.001 & $1.007 e-4$ & 3.188 \\
\hline$T+C+R P+T * R P+C * R P$ & 0.053 & $5.930 e-5$ & 0.001 & $7.597 e-5$ & 2.295 \\
\hline$T+C+R P+T * C+T * R P$ & 0.053 & $2.653 e-5$ & $4.775 e-4$ & $3.398 e-5$ & 2.433 \\
\hline$T+C+R P+T * C+T * R P+C * R P$ & 0.053 & $2.356 e-5$ & $4.241 e-4$ & $3.019 e-5$ & 6.678 \\
\hline$T+C+R P+T * C+T * R P+C * R P+R * C * R P$ & 0.053 & $6.359 e-6$ & $1.145 e-4$ & $8.147 e-6$ & 2.739 \\
\hline
\end{tabular}

Note. All models include PatientNr, Surgeon.T: MRI field strength (Tesla); C: MRI Contrast; RP: Repetition pair; $\mathrm{P}(\mathrm{M})$ : Prior model probability; $\mathrm{P}\left(\mathrm{M} \mid\right.$ data): posterior model probability; $\mathrm{BF}_{\mathrm{M}}$ : the change from prior odds to posterior odds; $\mathrm{BF}_{10}$ : the Bayes factor relative to the best model; error $\%$ : indicates the precision of the numerical approximation and it is thought that in many situations an error percentage below $20.0 \%$ is acceptable ${ }^{63}$. 
The distance between target coordinates $\sim$ planningsession and MRI contrasts
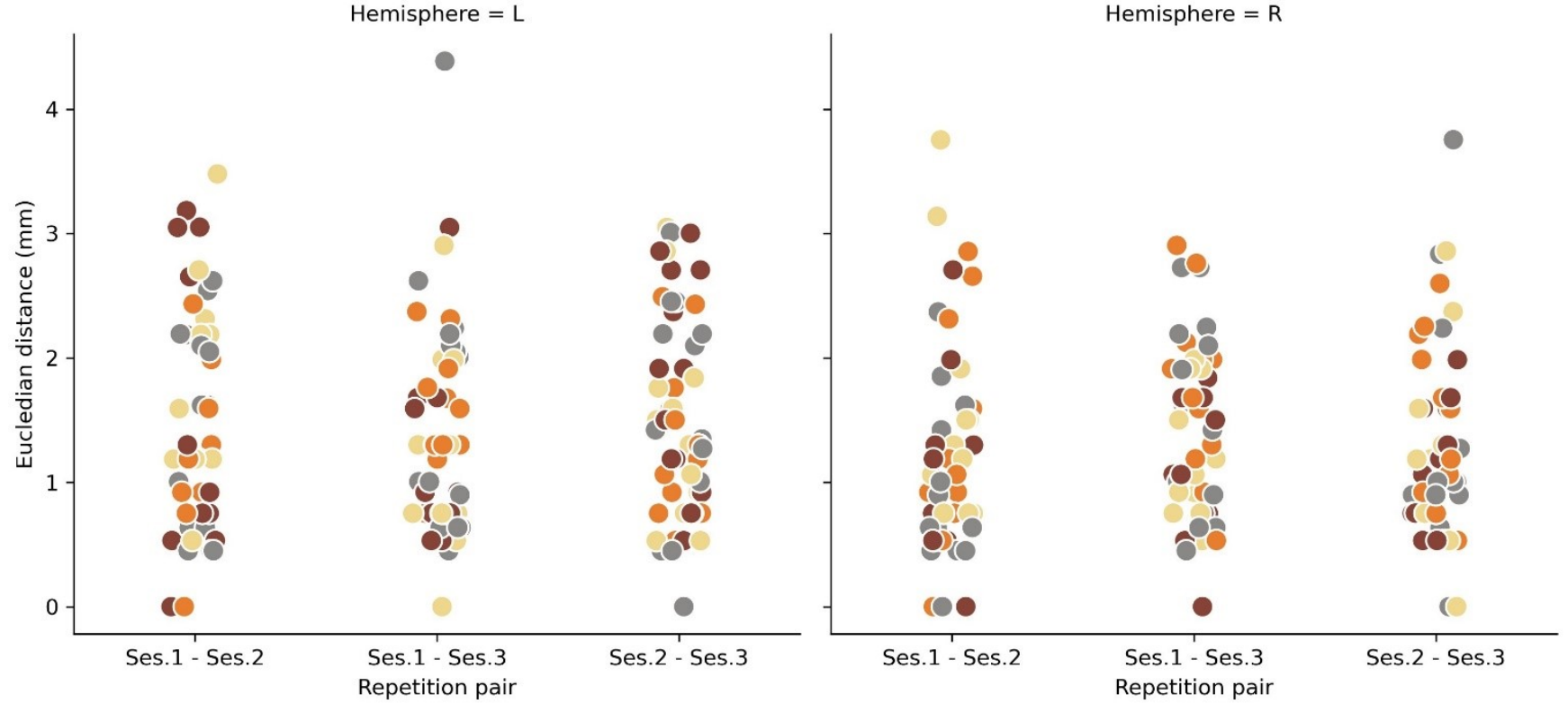

Contrast

- T2 (3T)

T2s (7T)

R2s (7T)

QSM (7T)

Figure 3. The Euclidean distance between the target coordinates over sessions and MRI contrasts. Note that we visualize the coordinates per hemisphere but as we had no apriori hypothesis on lateralization, hemisphere was not included as a factor in the statistical testing. 

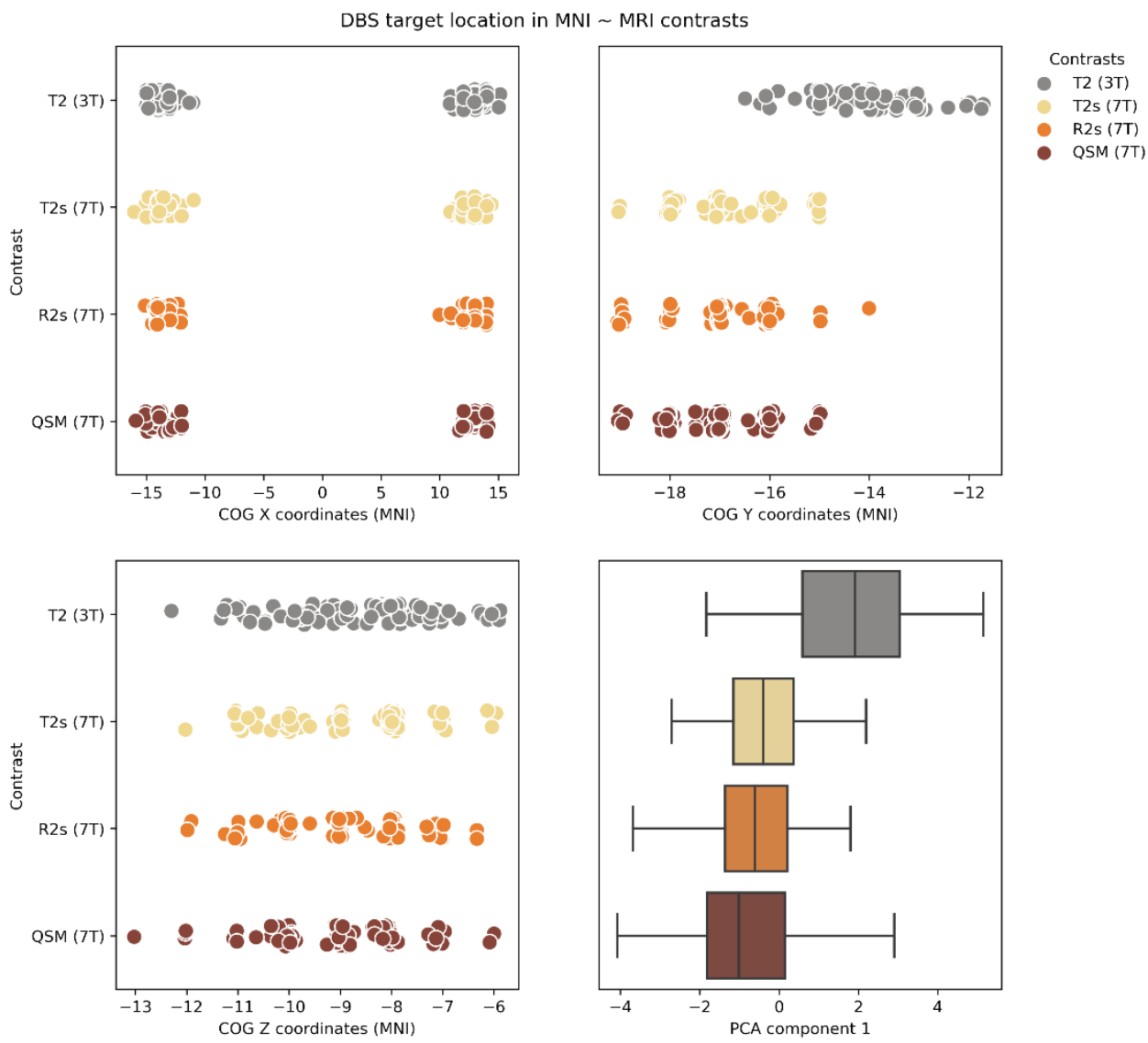

Figure 4. The intended DBS electrode location in MNI space over MRI contrasts. The left upper panel shows the $X$ MNI coordinates of the planned electrode's Centre of Gravity (COG) per MRI contrast, patient, surgeon and planning session registered from native to $\mathrm{MNI}$ space. Note that we visualize the $\mathrm{X}$ coordinates per hemisphere but as we had no a-priori hypothesis on lateralization, hemisphere was not included as a factor in the statistical testing. The right upper panel shows the Y MNI coordinates. The left lower panel shows the Z MNI coordinates. The right lower panel shows the boxplot of first PCA component per MRI contrast which were used for the statistical testing. 
Table 3. Model comparison of the spatial location of the DBS electrode targets.

\begin{tabular}{|c|c|c|c|c|c|}
\hline Models & $\mathrm{P}(\mathrm{M})$ & $\mathrm{P}(\mathrm{M} \mid$ data $)$ & $\mathrm{BF}_{\mathrm{M}}$ & $\mathrm{BF}_{10}$ & error \% \\
\hline $\bar{T}$ & 0.053 & 0.478 & 16.501 & 1.000 & \\
\hline$T+C$ & 0.053 & 0.274 & 6.804 & 0.574 & 5.736 \\
\hline$T+C+T * C$ & 0.053 & 0.130 & 2.682 & 0.271 & 4.329 \\
\hline C & 0.053 & 0.044 & 0.823 & 0.091 & 2.914 \\
\hline$T+R$ & 0.053 & 0.035 & 0.645 & 0.072 & 3.366 \\
\hline$T+C+R$ & 0.053 & 0.022 & 0.400 & 0.045 & 8.444 \\
\hline $\mathrm{T}+\mathrm{C}+\mathrm{R}+\mathrm{T} * \mathrm{C}$ & 0.053 & 0.009 & 0.170 & 0.020 & 4.263 \\
\hline$C+R$ & 0.053 & 0.003 & 0.059 & 0.007 & 3.861 \\
\hline$T+R+T * R$ & 0.053 & 0.002 & 0.045 & 0.005 & 3.162 \\
\hline$T+C+R+T * R$ & 0.053 & 0.001 & 0.026 & 0.003 & 4.968 \\
\hline$T+C+R+T * C+T * R$ & 0.053 & $7.006 \mathrm{e}-4$ & 0.013 & 0.001 & 6.743 \\
\hline $\mathrm{T}+\mathrm{C}+\mathrm{R}+\mathrm{C} * \mathrm{R}$ & 0.053 & $1.817 \mathrm{e}-4$ & 0.003 & $3.798 \mathrm{e}-4$ & 3.657 \\
\hline$T+C+R+T * C+C * R$ & 0.053 & $1.009 \mathrm{e}-4$ & 0.002 & $2.111 \mathrm{e}-4$ & 7.923 \\
\hline$T+C+R+T * R+C * R$ & 0.053 & $3.938 \mathrm{e}-5$ & $7.089 e-4$ & $8.234 \mathrm{e}-5$ & 21.787 \\
\hline$C+R+C * R$ & 0.053 & $3.166 e-5$ & $5.700 \mathrm{e}-4$ & $6.620 e-5$ & 3.088 \\
\hline$T+C+R+T * C+T * R+C * R$ & 0.053 & $1.859 \mathrm{e}-5$ & $3.346 \mathrm{e}-4$ & $3.886 e-5$ & 21.086 \\
\hline $\mathrm{T}+\mathrm{C}+\mathrm{R}+\mathrm{T} * \mathrm{C}+\mathrm{T} * \mathrm{R}+\mathrm{C} * \mathrm{R}+\mathrm{T} * \mathrm{C} * \mathrm{R}$ & 0.053 & $2.288 \mathrm{e}-6$ & $4.118 \mathrm{e}-5$ & $4.784 \mathrm{e}-6$ & 4.272 \\
\hline Null model (incl. PatientNr, Surgeon) & 0.053 & $1.506 e-41$ & $2.711 e-40$ & $3.149 e-41$ & 2.182 \\
\hline $\mathrm{R}$ & 0.053 & $6.767 e-43$ & $1.218 \mathrm{e}-41$ & $1.415 e-42$ & 2.807 \\
\hline
\end{tabular}

Note. All models include PatientNr, Surgeon.T: MRI field strength (Tesla); C: MRI Contrast; R: Repetition; P(M): Prior model probability; $\mathrm{P}\left(\mathrm{M} \mid\right.$ data): posterior model probability; $\mathrm{BF}_{\mathrm{M}}$ : the change from prior odds to posterior odds; $\mathrm{BF}_{10}$ : the Bayes factor relative to the best model; error \%: indicates the precision of the numerical approximation and it is thought that in many situations an error percentage below $20.0 \%$ is acceptable ${ }^{63}$.

As the amount of evidence to prefer the winning model over the second-best model was anecdotal, an analysis of effects was conducted (the results are given in Table 4). The BFincl indicates that the data is 7.24 times more likely under the models that include MRI field strength than models without this predictor. Whereas the BFincl indicates that the data is 2.98 times more likely under models that do not include MRI contrast as a predictor (1/0.336). This means that the target of the DBS electrode as quantified by the first component of the PCA differs between 3T and 7T MRI scans, where based on Figure 4, this difference seems to be mainly along the $\mathrm{Y}$-axis or in dorsal-ventral direction.

Table 4. Analysis of Effects - spatial location of the DBS electrode targets.

\begin{tabular}{lllrrr}
\hline Effects & $\mathbf{P}$ (incl) & $\mathbf{P}$ (excl) & $\mathbf{P}$ (incl|data) & $\mathbf{P}$ (excl|data) & $\mathbf{B F}_{\text {incl }}$ \\
\hline $\mathrm{T}$ & 0.737 & 0.263 & 0.953 & 0.047 & 7.236 \\
$\mathrm{C}$ & 0.737 & 0.263 & 0.485 & 0.515 & 0.336 \\
$\mathrm{R}$ & 0.737 & 0.263 & 0.074 & 0.926 & 0.029 \\
$\mathrm{~T} * \mathrm{C}$ & 0.316 & 0.684 & 0.140 & 0.860 & 0.352 \\
$\mathrm{~T} * \mathrm{R}$ & 0.316 & 0.684 & 0.005 & 0.995 & 0.010 \\
$\mathrm{C} * \mathrm{R}$ & 0.316 & 0.684 & $3.745 \mathrm{e}-4$ & 1.000 & $8.117 \mathrm{e}-4$ \\
$\mathrm{~T} * \mathrm{C} * \mathrm{R}$ & 0.053 & 0.947 & $2.288 \mathrm{e}-6$ & 1.000 & $4.118 \mathrm{e}-5$ \\
\hline
\end{tabular}

Note. T: MRI field strength (Tesla); C: MRI Contrast; R: Repetition; P(incl): prior inclusion probability; P(excl): prior exclusion probability; $\mathrm{P}(\mathrm{Incl} \mid$ data): posterior inclusion probability; $\mathrm{P}(\mathrm{excl} \mid$ data): posterior exclusion probability; BFincl: the inclusion Bayes factor. 


\section{Visibility of the STN}

To test whether the observed differences in MNI space might be explained by differences in STN visibility, the STN was parcellated by two raters and the Dice coefficient was calculated to quantify the interrater reliability. The mean Dice coefficient was 0.70 ( $S D=0.05)$ for $3 T$ and 0.61 (SD=0.09) for the 7T based masks. The Dice coefficient indicated moderate to substantial agreement between the two raters and were similar to our previous work ${ }^{56,64}$. The data was 4.95 (1/0.202) times more likely under the model with MRI field strength as a predictor compared to the Null model. In addition, we tested whether there were any differences in volume as quantified by the conjunction masks. The mean conjunction volume was $20.70 \mathrm{~mm}$ (SD=7.16) for the 3T and $15.09 \mathrm{~mm}$ (SD=2.47) for the 7T based masks. Note that the conjunction masks are considered extremely conservative volumetric estimates as only voxels that both raters agree on are included. The volumetric data was 2.93 (1/0.341) times more likely under the model with MRI field strength as a predictor than under the Null model.

\section{Discussion}

Direct targeting of the STN for DBS is shown to result in improved clinical outcome and has resulted in surgical centres to prefer it over an indirect targeting approach ${ }^{13,41,65}$. However, the feasibility and accuracy of direct targeting is dependent on the quality of the MRI image ${ }^{26,41}$. The current study assessed whether theoretical benefits of 7T MRI translate into more reliable targeting of the STN for DBS. We did so by comparing neurosurgical targets across field strength, image modality and across repetition using a test-retest approach. Target accuracy was assessed by calculating the distance in millimetres between the repetitive target locations. We hypothesized that optimized 7T image modalities would result in less variable target locations. Further, and in line with previous literature, we hypothesized that 7T-QSM images would result in the least variability in targeting compared to any other 7T images due to its superior ability in visualizing the STN.

The results, however, indicated that there was substantial evidence that the test-retest reliability of neurosurgeons is not influenced by MRI field strength, contrast or targeting session. This indicates that the neurosurgeons selected the same target site within a given MRI contrast across sessions. It can therefore be argued that variability based on direct targeting methods probably is not a factor on itself in suboptimal placement of the DBS lead, since the same target site would have been selected if targeting was performed repetitively.

Next to the variability in stereotactic planning, the exact anatomical location of the electrode target may potentially on itself be a factor in suboptimal placement of the DBS lead. The general consensus is that the effectiveness of DBS depends on the portion of the STN in which the DBS lead is placed, with the dorsolateral portion of the STN being most 
effective in treating PD ${ }^{25,38-40}$. A second question that was addressed is whether the neurosurgeons select the similar target sites between MRI contrasts and field strengths, considering that different contrasts and resolutions might convey different anatomical information $43,44,66$. While the neurosurgeons were stable in selecting the electrode location, the location itself seemed to differ between field strengths whereby the selected electrode location appeared (mainly) more ventral when using a 3T MRI image versus a 7T MRI image. This shift in location is unlikely to have occurred due to a difference in STN visibility as both $3 \mathrm{~T}$ and $7 \mathrm{~T}$ resulted in moderate to substantial interrater agreement. As such it does not seem that neurosurgeons are hampered by reduced visibility of the STN but it might be the case that they use different image features, such as landmarks, to determine the electrode location. Our results are in line with the recent work by ${ }^{24}$ where it was shown that the intended DBS electrode sites were more posterior and inferior to the midcommissural point when using 1.5T and 3T compared to 7T MRI images (but see ${ }^{67}$ ).

Whether the electrode is placed more ventral has clear clinical relevance as previous work has indicated that more ventral stimulation seems to be associated with reduced cognitive outcome ${ }^{8,41,68}$. For example, it was shown that stimulation of specifically the ventral STN led to an impaired performance on the Go-No-Go task, which requires higher cognitive functions ${ }^{69}$. Our results showed that the selected electrode location using a 3T MRI image is more ventral compared to using a 7T MRI image. Future work should focus on whether this theoretical difference in STN targeting based on MRI strength actually leads to less cognitive and psychiatric side-effects. It should further be studied what differences in imaging features causes the difference in electrode location when targeting on 7T versus 3T MRI images.

There are a number of limitations to the present study. The number of patients that were included in the study was limited, but we feel that this is a minor issue as the main metric of interest was the test-retest reliability within a patient and that direct pre-operative planning approaches always employ individualized targeting ${ }^{27}$. Another limitation is that the selection of MRI contrasts included a standard clinical 3T protocol and an optimized 7T protocol, adapted for anatomical changes with both age and disease. We did not however include either a 7T-T2 or an optimized/quantitative 3T-T2* based sequence which would have allowed for a direct comparison between field strengths while directly accounting for difference in MRI contrasts mechanism. As such, it remains challenging to disentangle the contributions of MRI contrast and MRI field strength in the difference in MNI target location. We attempted to quantify the different factors by conducting an analysis of effects where the results indicated that the data is 6.62 times more likely to occur in models that include MRI field strength than not, and that the data is 2.93 times more likely to occur under models that did not include MRI contrast as a predictor. Together with the findings reported by ${ }^{24}$ we would tentatively interpret our results as evidence in favour of an effect 
of MRI field strength on the intended electrode position and not so much due to a difference in MRI contrast mechanisms. A final limitation that complicates the interpretability of the results in standard MNI space are the potential biases in $\mathrm{MNI}$ registrations for the $3 \mathrm{~T}$ data compared to the $7 \mathrm{~T}$ scans due to the difference in voxel geometry and volume ${ }^{70,71}$.

In light of these limitations, the present study provides substantial evidence that regardless of the MRI field strength and MRI contrast, neurosurgeons are stable in selecting the intended DBS electrode location. In addition, we conclude that the intended electrode location differs between MRI field strengths, where the 3T scans resulted in a more ventral location. Future research should focus on what image features drive the neurosurgeons to select a slightly different location across the images. 


\section{References}

1. Benabid, A. L., Chabardes, S., Mitrofanis, J. \& Pollak, P. Deep brain stimulation of the subthalamic nucleus for the treatment of Parkinson's disease. Lancet Neurol 8, 67-81 (2009).

2. Limousin, P. et al. Effect of parkinsonian signs and symptoms of bilateral subthalamic nucleus stimulation. Lancet 345, 91-95 (1995).

3. Deuschl, G. et al. A randomized trial of deep-brain stimulation for Parkinson's disease. N Engl J Med 355, 896-908 (2006).

4. Limousin, P. \& Foltynie, T. Long-term outcomes of deep brain stimulation in Parkinson disease. Nat Rev Neurol 15, 234-242 (2019).

5. Frank, M. J., Samanta, J., Moustafa, A. A. \& Sherman, S. J. Hold your horses: impulsivity, deep brain stimulation, and medication in parkinsonism. Science 318, 1309-1312 (2007).

6. Parsons, T. D., Rogers, S. A., Braaten, A. J., Woods, S. P. \& Tröster, A. I. Cognitive sequelae of subthalamic nucleus deep brain stimulation in Parkinson's disease: a meta-analysis. The Lancet Neurology 5, 578-588 (2006).

7. Temel, Y. et al. Behavioural changes after bilateral subthalamic stimulation in advanced Parkinson disease: a systematic review. Parkinsonism Relat Disord 12, 265-272 (2006).

8. Zarzycki, M. Z. \& Domitrz, I. Stimulation-induced side effects after deep brain stimulation - a systematic review. Acta Neuropsychiatrica 32, 57-64 (2020).

9. Gilmore, G., Lee, D. H., Parrent, A. \& Jog, M. The current state of postoperative imaging in the presence of deep brain stimulation electrodes: Imaging in the Presence of DBS Electrodes. Mov Disord. 32, 833838 (2017).

10. Kloc, M., Kosutzka, Z., Steno, J. \& Valkovic, P. Prevalent placement error of deep brain stimulation electrode in movement disorders (technical considerations). Bratisl Lek Listy 118, 647-653 (2017).

11. Petry-Schmelzer, J. N. et al. Non-motor outcomes depend on location of neurostimulation in Parkinson's disease. Brain 142, 3592-3604 (2019).

12. Giller, C. A. \& Jenkins, P. Some technical nuances for deep brain stimulator implantation. Interdisciplinary Neurosurgery 2, 29-39 (2015).

13. Lahtinen, M. J. et al. A comparison of indirect and direct targeted STN DBS in the treatment of Parkinson's disease-surgical method and clinical outcome over 15-year timespan. Acta Neurochir (Wien) 162, 1067-1076 (2020).

14. Bus, S. et al. Borders of STN determined by MRI versus the electrophysiological STN. A comparison using intraoperative CT. Acta Neurochir (Wien) 160, 373-383 (2018).

15. Verhagen, R. et al. Comparative study of microelectrode recording-based STN location and MRI-based STN location in low to ultra-high field (7.0 T) T2-weighted MRI images. J Neural Eng 13, 066009 (2016).

16. Deistung, A. et al. Toward in vivo histology: A comparison of quantitative susceptibility mapping (QSM) with magnitude-, phase-, and R2*-imaging at ultra-high magnetic field strength. Neurolmage 65, 299314 (2013).

17. Hollander, G. de et al. A gradual increase of iron toward the medial-inferior tip of the subthalamic nucleus. Human Brain Mapping 35, 4440-4449 (2014).

18. Habets, J., Isaacs, B., Vinke, S. \& Kubben, P. Controversies in Deep Brain Stimulation Surgery: MicroElectrode Recordings. in Evidence for Neurosurgery: Effective Procedures and Treatment (eds. Bartels, R. H. M. A., Rovers, M. M. \& Westert, G. P.) 97-109 (Springer International Publishing, 2019). doi:10.1007/978-3-030-16323-5_8.

19. Forstmann, B. U., Isaacs, B. R. \& Temel, Y. Ultra High Field MRI-Guided Deep Brain Stimulation. Trends Biotechnol 35, 904-907 (2017).

20. Isaacs, B. R. et al. Methodological Considerations for Neuroimaging in Deep Brain Stimulation of the Subthalamic Nucleus in Parkinson's Disease Patients. J Clin Med 9, (2020). 
21. Inglese, M., Fleysher, L., Oesingmann, N. \& Petracca, M. Clinical applications of ultra-high field magnetic resonance imaging in multiple sclerosis. Expert Rev Neurother 18, 221-230 (2018).

22. Keuken, M. C., Isaacs, B. R., Trampel, R., van der Zwaag, W. \& Forstmann, B. U. Visualizing the Human Subcortex Using Ultra-high Field Magnetic Resonance Imaging. Brain Topogr 31, 513-545 (2018).

23. Bot, M. et al. Deep brain stimulation for Parkinson's disease: defining the optimal location within the subthalamic nucleus. J Neurol Neurosurg Psychiatry 89, 493-498 (2018).

24. Bot, M. et al. Defining the Dorsal STN Border Using 7.0-T MRI: A Comparison to Microelectrode Recordings and Lower Field Strength MRI. Stereotact Funct Neurosurg 97, 153-159 (2019).

25. Duchin, Y. et al. Patient-specific anatomical model for deep brain stimulation based on 7 Tesla MRI. PLoS One 13, e0201469 (2018).

26. Hartmann, C. J., Fliegen, S., Groiss, S. J., Wojtecki, L. \& Schnitzler, A. An update on best practice of deep brain stimulation in Parkinson's disease. Ther Adv Neurol Disord 12, 1756286419838096 (2019).

27. Isaacs, B. R. et al. 3 versus 7 Tesla magnetic resonance imaging for parcellations of subcortical brain structures in clinical settings. PLOS ONE 15, e0236208 (2020).

28. Springer, E. et al. Comparison of Routine Brain Imaging at 3 T and 7 T. Invest Radiol 51, 469-482 (2016).

29. Chavhan, G. B., Babyn, P. S., Thomas, B., Shroff, M. M. \& Haacke, E. M. Principles, techniques, and applications of T2*-based MR imaging and its special applications. Radiographics 29, 1433-1449 (2009).

30. Elolf, E. et al. Improved visibility of the subthalamic nucleus on high-resolution stereotactic MR imaging by added susceptibility $(\mathrm{T} 2 *$ ) contrast using multiple gradient echoes. AJNR Am J Neuroradiol 28, 10931094 (2007).

31. Plantinga, B. B. A clear view on Parkinson's disease : 7T MRI investigation of the basal ganglia and development of patient-specific deep brain stimulation. in (2016).

32. Ulla, M. et al. Is R2* a new MRI biomarker for the progression of Parkinson's disease? A longitudinal follow-up. PLoS One 8, e57904 (2013).

33. Schäfer, A. et al. Direct visualization of the subthalamic nucleus and its iron distribution using highresolution susceptibility mapping. Human Brain Mapping 33, 2831-2842 (2012).

34. Zhou, D., Liu, T., Spincemaille, P. \& Wang, Y. Background field removal by solving the Laplacian boundary value problem. NMR Biomed 27, 312-319 (2014).

35. Alkemade, A. et al. Comparison of T2*-weighted and QSM contrasts in Parkinson's disease to visualize the STN with MRI. PLoS One 12, e0176130 (2017).

36. Horn, A. et al. Connectivity Predicts deep brain stimulation outcome in Parkinson disease. Ann Neurol 82, 67-78 (2017).

37. Vanegas-Arroyave, N. et al. Tractography patterns of subthalamic nucleus deep brain stimulation. Brain 139, 1200-1210 (2016).

38. Hamel, W. et al. Targeting of the Subthalamic Nucleus for Deep Brain Stimulation: A Survey Among Parkinson Disease Specialists. World Neurosurg 99, 41-46 (2017).

39. Starr, P. A. Placement of deep brain stimulators into the subthalamic nucleus or Globus pallidus internus: technical approach. Stereotact Funct Neurosurg 79, 118-145 (2002).

40. Welter, M.-L. et al. Optimal target localization for subthalamic stimulation in patients with Parkinson disease. Neurology 82, 1352-1361 (2014).

41. Machado, A. et al. Deep brain stimulation for Parkinson's disease: Surgical technique and perioperative management. Movement Disorders 21, 12 (2006).

42. Horn, A. et al. Lead-DBS v2: Towards a comprehensive pipeline for deep brain stimulation imaging. Neurolmage 184, 293-316 (2019).

43. Bazin, P.-L., Alkemade, A., Mulder, M. J., Henry, A. G. \& Forstmann, B. U. Multi-contrast anatomical subcortical structures parcellation. eLife 9, e59430 (2020).

44. Visser, E. et al. Automatic segmentation of the striatum and globus pallidus using MIST: Multimodal Image Segmentation Tool. Neurolmage 125, 479-497 (2016). 
45. Visser, E., Keuken, M. C., Forstmann, B. U. \& Jenkinson, M. Automated segmentation of the substantia nigra, subthalamic nucleus and red nucleus in 7T data at young and old age. Neurolmage 139, 324-336 (2016).

46. Caan, M. W. A. et al. MP2RAGEME: T1, T2 *, and QSM mapping in one sequence at 7 tesla. Hum Brain Mapp 40, 1786-1798 (2018).

47. Marques, J. P. et al. MP2RAGE, a self bias-field corrected sequence for improved segmentation and T1mapping at high field. Neuroimage 49, 1271-1281 (2010).

48. Eckstein, K. et al. Computationally Efficient Combination of Multi-channel Phase Data From Multi-echo Acquisitions (ASPIRE). Magn Reson Med 79, 2996-3006 (2018).

49. Jenkinson, M., Beckmann, C. F., Behrens, T. E. J., Woolrich, M. W. \& Smith, S. M. FSL. Neuroimage 62, 782-790 (2012).

50. Smith, S. M. Fast robust automated brain extraction. Hum Brain Mapp 17, 143-155 (2002).

51. Whittall, K. P. et al. In vivo measurement of T2 distributions and water contents in normal human brain. Magn Reson Med 37, 34-43 (1997).

52. Li, W., Avram, A. V., Wu, B., Xiao, X. \& Liu, C. Integrated Laplacian-based phase unwrapping and background phase removal for quantitative susceptibility mapping. NMR Biomed 27, 219-227 (2014).

53. Li, W. et al. A method for estimating and removing streaking artifacts in quantitative susceptibility mapping. Neuroimage 108, 111-122 (2015).

54. van Bergen, J. M. G. et al. Quantitative susceptibility mapping suggests altered brain iron in premanifest Huntington's disease. AJNR Am J Neuroradiol 37, 789-796 (2016).

55. Liberti, L., Lavor, C., Maculan, N. \& Mucherino, A. Euclidean Distance Geometry and Applications. SIAM Rev. 56, 3-69 (2014).

56. Keuken, M. C. et al. Effects of aging on $T_{1}, T_{2}{ }^{*}$, and QSM MRI values in the subcortex. Brain Struct Funct 222, 2487-2505 (2017).

57. Dice, L. R. Measures of the Amount of Ecologic Association Between Species. Ecology 26, 297-302 (1945).

58. JASP Team. JASP (Version 0.14.1). (2020).

59. Morey, R. D. \& Rouder, J. N. BayesFactor (Version 0.9.10-2). (2015).

60. Rouder, J. N., Morey, R. D., Speckman, P. L. \& Province, J. M. Default Bayes factors for ANOVA designs. Journal of Mathematical Psychology 56, 356-374 (2012).

61. Jeffreys, S. H. The Theory of Probability. (Oxford University Press, 1998).

62. Wetzels, R. et al. Statistical Evidence in Experimental Psychology: An Empirical Comparison Using 855 t Tests. Perspect Psychol Sci 6, 291-298 (2011).

63. Bergh, D. van den et al. A Tutorial on Conducting and Interpreting a Bayesian ANOVA in JASP. LAnnee psychologique Vol. 120, 73-96 (2020).

64. Landis, J. R. \& Koch, G. G. The Measurement of Observer Agreement for Categorical Data. Biometrics 33, 159-174 (1977).

65. Tonge, M., Kocabicak, E., Ackermans, L., Kuijf, M. \& Temel, Y. Final Electrode Position in Subthalamic Nucleus Deep Brain Stimulation Surgery: A Comparison of Indirect and Direct Targeting Methods. Turk Neurosurg 26, 900-903 (2016).

66. McRobbie, D. W. MRI from picture to proton. (Cambridge University Press, 2006).

67. van Laar, P. J. et al. Surgical Accuracy of 3-Tesla Versus 7-Tesla Magnetic Resonance Imaging in Deep Brain Stimulation for Parkinson Disease. World Neurosurgery 93, 410-412 (2016).

68. McNeely, M. et al. Effects of deep brain stimulation of dorsal versus ventral subthalamic nucleus regions on gait and balance in Parkinson disease. J Neurol Neurosurg Psychiatry 82, 1250-1255 (2011).

69. Hershey, T. et al. Mapping Go-No-Go performance within the subthalamic nucleus region. Brain 133, 3625-3634 (2010).

70. Mulder, M. J., Keuken, M. C., Bazin, P.-L., Alkemade, A. \& Forstmann, B. U. Size and shape matter: The impact of voxel geometry on the identification of small nuclei. PLoS One 14, (2019). 
71. Zhao, C., Carass, A., Jog, A. \& Prince, J. L. Effects of Spatial Resolution on Image Registration. Proc SPIE Int Soc Opt Eng 9784, (2016). 


\section{Chapter 8}

\section{General discussion}



Parkinson's disease (PD) not only has a huge impact on the patient and his or her loved ones, but also has a significant effect on society. This neurodegenerative disease is a common condition, with a rapidly increasing incidence and prevalence in the past twenty years ${ }^{1}$. The aim of this thesis was to develop and to work further on methods for PD treatment and for the diagnostic work-up of PD. More specifically, the aim was to 1) find out which role wearable sensors can play in tracking and treating PD and 2) find out what role MRI can play in tracking and treating PD. In this final chapter, the findings of this thesis will be summarized and discussed, and future perspectives on patient research and clinical relevance will be sketched.

\section{Wearable sensors can play an important role in adaptive deep brain stimulation}

Chapter 2 provides a narrative review on adaptive deep brain stimulation (aDBS) for PD. The idea behind aDBS is that the system automatically adapts its stimulation parameters based on the clinical state of the patient. Stimulation will be provided when PD symptom expression is (severely) present, while less or no stimulation will be provided when symptom expression is negligible. Chapter $\mathbf{2}$ focuses on the use of wearable sensors as input signal for aDBS. Important progress has been made on the use of wearable sensors in predicting and detecting PD symptoms and the first results of studies showing wearables-based aDBS have been published ${ }^{2-4}$. Malekmohammadi et al., (2016) were the first ones evaluating the safety, tolerability, and feasibility of a fully portable aDBS system in five tremor-dominant PD patients. The power of resting tremor was measured by a wearable sensor and stimulation was turned on when tremor power exceeded a pre-set threshold. The system showed to be safe, tolerable, effective, and efficient. The study by Malekmohammadi et al., (2016) and other studies regarding wearables-based aDBS were however all based on tremor detection, while no other PD symptoms were implemented in the aDBS system. While tremor-dominant PD patients may benefit from wearablesbased aDBS, the applicability for rigid-akinetic PD patients remains unclear. To be suitable for rigid-akinetic PD patients as well, improvement should be made in the algorithms for monitoring PD symptoms other than tremor. Further concerns of wearables-based aDBS are the difficulty to distinguish voluntary movements from PD symptoms, the need for patients to wear sensors almost chronically, and signal processing and wireless data transmission may limit the battery life of both wearables and pulse generators. Ideally, one would have a system with one or more aesthetically attractive and small wearable sensors, which process and transmit data wirelessly to the pulse generator, which in turn adapts the stimulation parameters. With the current developments, this pulse generator might even be a rechargeable one ${ }^{5}$.

Although multiple studies have focused on aDBS, there are still various unsolved issues. It might be that various input signals, like wearable sensors and local field potentials, should 
be combined to be able to serve as valuable input signal for aDBS. A recent study for example paired measured field potentials with data from wearable sensors to decode PD states of inadequate or excessive movement, which were later used for aDBS ${ }^{6}$. This study showed, that combining data of various input signals can be beneficial for the development of a valuable input signal for aDBS. For tremor-dominant patients, clinical trials with longer follow-up periods are needed to prove whether wearables-based aDBS systems are superior to continuous DBS systems regarding for example battery life and stimulation induced side-effects. Future work could also focus on the stimulation parameter modulation in aDBS. Most studies modulate the amplitude, but the potential of other parameter adaptations, like frequency and phase, could also be studied.

Another aspect in which aDBS systems have potential is to incorporate the subjective experience of motor symptoms and the assessment of non-motor symptoms. Non-motor symptoms are important for the quality-of-life scores and in predicting overall DBS outcomes ${ }^{7}$. Stimulation at a level which totally suppresses tremor might for example lead to unwanted speech disturbances. Mobile Health applications are considered as a valuable tool which can be integrated into PD care and which collect information about non-motor PD symptoms and side effects. Not only can mobile Health applications be used for regular PD patient care, they can also be used as input for manual DBS setting adjustments ${ }^{8}$, and even hold promise as additional input signal for aDBS.

\section{Multimodal monitoring systems are feasible to use for Parkinson's disease patients}

Multimodal monitoring systems combine mobile Health applications with wearable sensors. Such systems have the potential to eventually serve as input for aDBS, but also hold promise to be used as a general home monitoring system for PD patient care. Nowadays, the disease course is charted by a clinician during visits to the outpatient clinic. This is associated with limitations; 1 ) The assessment only permits a snapshot of the clinical situation, whereas symptoms generally fluctuate and might include recall bias, since patients have to recall their clinical situation of the past months. 2) The assessment has a subjective character. 3) PD patients might perform better than on average during in-clinic assessments for example because patients can better express themselves when they are in their ON-phase. 4) The assessment is time-consuming both for the patient and clinician during a busy regular outpatient clinic. Because of these reasons multimodal monitoring systems, providing more frequent and objective ratings of symptoms and the disease course, are essential to obtain a better treatment for PD patients.

In Chapter $\mathbf{3}$ the feasibility study of a newly developed multimodal PD monitoring system is described. This system combines wearable sensors measuring acceleration and rotational acceleration with an experience sampling method (ESM) application. This 
combination was often suggested in PD ${ }^{9,10}$, but has never been tested before. The feasibility of the new monitoring system was therefore studied in PD patients during their daily life. Twenty PD patients participated in this study. During a period of two consecutive weeks, participants had to wear three wearable sensors (two wrist sensors and a chest sensor) and had to complete questionnaires at seven semi-random moments per day on their mobile phone. The questionnaires contained questions about motor and non-motor PD symptoms as well as side-effects. Wearable sensors collected objective movement data, and the questionnaires collected subjective scores.

To get an idea of the feasibility of the monitoring system the following measures were evaluated; 1) How much time the sensors were worn by the participants. 2) How much of the ESM questionnaires were completed. 3) How were the evaluation scores of the two week usage of the system. In short, the results showed that the participants wore the wearable sensors during $94 \%$ of the instructed timeframe and even beyond. Furthermore, questionnaire completion rates were high $(79,1 \%)$ and participants evaluated the monitoring system positively. The high percentage of wearing time, in combination with the high completion rates and positive evaluation of the monitoring system proved the system to be feasible. For longer use the monitoring system may be too intense and wearing comfort of the wearables needs to be optimized. Based on the results of this feasibility study, it can be expected that a monitoring system which has less frequent questionnaires, or less (only patient specific) questions per questionnaire, and which has more comfortable wearable sensors can be easily used by PD patients for over a month. This offers huge potential for tracking the PD course. PD patients could for example use the monitoring system at home during the month preceding their visit to the outpatient clinic, to offer the clinician a valid inside in their PD course.

\section{Multimodal monitoring systems: a new combination of data}

Chapter 3 also provides a proposition for data processing of the newly developed monitoring system. It should be noted that the combination of wearable sensor data with ESM data was completely new, so therefore a data processing proposition was provided. In addition, first results were shown, since it was unknown if the objective wearable sensor data could be combined with, and eventually predict, the subjective ESM scores. In short, it was proposed that a fifteen minute window of sensor data prior to a completed questionnaire will be extracted. This timeframe can be divided into windows of a certain length, from which different features in the time and spectral domain will be extracted. These features, like for example amplitude of the signal or power of a certain frequency, can then be related to the corresponding answers of the ESM questionnaire. See Figure 5 of Chapter $\mathbf{3}$ for an overview of the proposed data processing steps. 
In this thesis, two analyses are described which investigated whether ESM answers can be employed as a ground truth for the sensor data. In Chapter 3 it was evaluated whether OFF moments (as indicated by one participant with severe ON/OFF fluctuations) can be predicted based on features calculated from the sensor data 15 minutes prior to the completed questionnaire. This yielded a reliable detection of subjectively registered OFF moments based on sensor data using a logistic regression classifier, with the area under the curve being 0.73. In Chapter 4 it was evaluated whether the subjective tremor score (as indicated by one tremor-dominant participant with fluctuations in tremor severity) can be predicted from the sensor data. In addition, it was evaluated what window length was most beneficial for feature extraction and further analysis. Standard features from the literature were used for this analysis ${ }^{11-13}$. The results showed that the correlation coefficients between the original and predicted tremor scores were significantly above chance level for all window lengths except for the 120 second long windows. The highest correlation scores $(r=0.43)$ were obtained for a 15 minute long window. It could therefore be concluded that for the analyses of this individual patient, longer window lengths seemed to result in a better prediction quality. This study showed that we can predict the subjective tremor score given by this patient, based on parameters extracted from wearable sensor data 15 minutes prior to the completed questionnaire.

\section{Future perspectives of using wearable sensors to track and treat PD}

The capability and willingness of PD patients using home monitoring systems will increase as a result of the overall increase in smartwatch and smartphone usage. The study presented in Chapter 3 provides evidence that the current PD population is ready to use home monitoring systems like the one presented. Also, the development of algorithms able to calculate PD symptom scores will become more accurate and quicker when research continues, and therefore the validation of wearable sensor data will be more convincing. This certainly shows potential of home monitoring systems to serve in both PD monitoring for general treatment and eventually for aDBS.

One of the pitfalls in this field of research is that studies are performed, while not being shared publicly afterwards. Research groups for example are using algorithms which are not openly available to other research groups, or are using wearable sensors with built-in algorithms ${ }^{14}$. By sharing algorithms (open science), research will become more reproducible, and duplication can be avoided. In addition to the algorithms, the availability of data can also save work all over the world. The strength of our feasibility study is that the data presented in Chapter $\mathbf{3}$ and Chapter $\mathbf{4}$ is openly available to other research groups 15 , so it can be used for example for the development of new algorithms without having to perform the intense data collection again. 
In sum, there are still challenges to overcome before the translation from research to the clinic can be made. It is however a matter of time before PD home monitoring is a standard option in PD care. All factors needed are currently in development; 1). Increased willingness of PD patients to wear and/or use smartwatches/smartphones. 2). Development of smaller/more attractive wearable sensors with increased power. 3). Development and validation of more accurate algorithms.

\section{MRI as indispensable study method for PD}

PD is a neurodegenerative disease, resulting in abnormalities in the brain which occur according to the well accepted Braak stadia of PD pathology ${ }^{16}$. Because of this brain pathology occurring in PD, MRI has become an indispensable study method often used in PD research over the past decades. Unfortunately, most of the time small groups are studied at only one moment in time using lower field MRI. To better understand PD, we designed the first longitudinal, observational, ultra-high field imaging (7T) study in early PD patients; the TRACK-PD study. Chapter 5 provides the detailed protocol of this study. In short, the TRACK-PD study aims to: 1) Identify distinctive MRI characteristics in PD which differ from healthy controls and to create a diagnostic tool based on these characteristics. 2) To correlate MRI characteristics to clinical phenotype, genetics and progression of symptoms. 3) To detect MRI characteristics for disease progression which could eventually be valuable for the evaluation of new therapies. The TRACK-PD study aims to include a cohort of 130 early PD patients (diagnosed with PD less than 3 years ago) and 60 healthy controls. The study consists of three testing days for all participants, one at baseline, one after two years, and one after four years. During each testing day structural 7T MRI scans are made, consisting of a T1, T2*, neuromelanin and diffusion weighted scan. In addition a resting-state functional MRI scan is made. Besides the MRI, an assessment of motor, cognitive, neuropsychiatric and autonomic symptoms is performed using wearable sensors, validated questionnaires and rating scales. At baseline a blood sample is collected.

\section{T olfactory tract diffusion measures cannot track PD}

The decreased ability to smell and to detect odours is one of the classical early symptoms of PD. The pathological mechanism is poorly understood but possibly involves neurodegeneration of the olfactory tract (OT) ${ }^{17}$. In fact, from a neuropathological point of view, the OT and olfactory bulb are often recognized as one of the first structures affected, according to the Braak stadia of PD pathology ${ }^{16}$. Previously, a meta-analysis highlighted that the OT could potentially serve as a biomarker for PD ${ }^{18}$. However, previous work studying diffusion measures of the OT or olfactory areas showed varying results. In Chapter 6 it was therefore evaluated whether previously identified differences in diffusion measures of the OT between PD patients and healthy controls could be replicated. This 
project used a subset of data of the TRACK-PD study (collected in 2019 and 2020), resulting in data of 62 early PD patients and 27 healthy controls.

A whole brain diffusion weighted scan was acquired of each participant. These scans were pre-processed and manual seed regions of interest were drawn in the OT region by two researchers. Next, tractography of the OT was performed and mean diffusion measures (fractional anisotropy, mean diffusivity, radial diffusivity, and axial diffusivity) of the generated OT fiber tracts were calculated.

Results showed no differences in diffusion measures of the OT between early PD patients and healthy controls. Further, results did not show any significant correlations for the PD group between MDS-UPDRSIII (ON medication), disease duration, SCOPA-AUT, and RBDSQ scores and diffusion measures of the OT. The results of this study did not support our hypothesis that diffusion measures of the OT differ between PD patients and healthy controls. Compared to previous work which also used OT tractography of diffusion weighted images ${ }^{19}$, there are two main differences. One is the difference in MRI field strength used (3T versus 7T) and one is the assessment of smell capabilities. Our use of 7T MRI data may result in more precise targeting of the OT, however the actual gain of using 7T over 3T is not that clear for diffusion weighted scans ${ }^{20}$, especially since the OT is close to air filled sinuses, which make this region more prone to artifacts. Further, the PD group of previous work ${ }^{19}$ had a decreased ability to smell and to detect odours compared to their healthy control group. Using an unselected group of PD patients (not selected based on smell level), may also contribute to the fact that we did not find any differences in diffusion measures of the OT between groups.

In conclusion, this study showed that fiber tracking of the OT using ultra-high field 7T MRI data was feasible for both the early PD patients and healthy controls. Further, we concluded that diffusion measures of the OT unfortunately were not useful as early clinical biomarker for PD. Based on the OT being one of the first structures affected in PD, it still has potential to serve as an early clinical biomarker region for PD. Future work is needed to unravel whether other MRI measurements of the OT differ between early PD patients and healthy controls. In addition, it could be studied whether diffusion measures of other affected brain structures, or a combination of these, can serve as a biomarker for PD.

\section{MRI field strength does affect the spatial location of a deep brain stimulation target}

Ultra-high-field MRI systems (7T and above) can obtain submillimeter anatomical information with increased contrast ${ }^{21,22}$. As described in the previous paragraph, this can be used for the search to diagnostic biomarkers for PD. Alternatively, the increased submillimeter anatomical information can also be used in anatomical targeting for deep brain stimulation (DBS) in PD. In Chapter $\mathbf{7}$ it was evaluated whether theoretical benefits of ultra-high field 7T MRI translate in to more accurate targeting of the subthalamic nucleus 
for DBS in PD. Three DBS-experienced neurosurgeons targeted the optimal subthalamic nucleus site for DBS in PD. This was done on the left and right side of three repetitions of 3T-T2, 7T-T2*, 7T-R2* and 7T-QSM images for five PD patients, resulting in a total of 120 targets per neurosurgeon.

To assess the target accuracy both across and within subject space, the distance between the three repetitive coordinates was used as index of variability of targeting. Distances were compared between field strength, image type and repetition. In addition, images and coordinates were registered to MNI space (a standardized anatomical MRI dataset), and the anatomical coordinates were compared between field strength, image type and repetition.

Results indicated that the test-retest reliability of the target coordinates were not affected by field strength, image type or repetition. Neurosurgeons are therefore stable in selecting the DBS target site across field strength, image type and targeting sessions. Analysis of the coordinates in $\mathrm{MNI}$ space however revealed that field strength does affect the spatial location of the DBS electrode targets. More specifically, the actual selected location of the electrode is seemingly more ventral when using the $3 \mathrm{~T}$ scan compared to the $7 \mathrm{~T}$ scans. This is interesting, since previous work has indicated that more ventral stimulation of the subthalamic nucleus seems to be associated with reduced cognitive outcomes ${ }^{23-25}$. It can therefore be hypothetically argued that using 7T MRI images may lead to less cognitive and psychiatric side-effects because of selecting a less ventral electrode location. It is unclear what causes these differences in electrode location when targeting on 7T versus 3T clinical images and what effect this different location has on DBS efficacy in PD, which could be an interesting topic for further research.

\section{Future perspectives of using MRI to track and treat PD}

The studies described in Chapter 6 and Chapter 7 both elaborate on the potential use of ultra-high field MRI to track and treat PD. Chapter 6 provides a project focussing on tracking PD by studying one of the many proposed biomarkers for PD. Diffusion measures of the OT did not show any differences between the PD group and healthy controls. Since a difference between groups is required to serve as a valuable biomarker, it was concluded that 7T diffusion measures of the OT are unusable as biomarker for PD. Other studies previously focussed on comparing diffusion measures of the substantia nigra between PD patients and healthy controls, but also for the substantia nigra results heavily differed between studies ${ }^{26,27}$.

Overall there are quite some groups studying potential imaging biomarkers, but to my knowledge, none of them found a biomarker which is able to distinguish PD patients from healthy controls and which is consistently replicated by other groups as well. One of the pitfalls here is that so-called 'negative outcomes', like the ones presented in Chapter 6, 
sometimes are hard to publish or are not even tried to be published since they are not considered interesting enough. Work can however be saved by also publishing these 'negative outcomes'. By using more precise 7T images we and/or other research groups may be able to find steady differences between PD patients and healthy controls which can be used as biomarker. As highlighted by a recent study, these differences might for example be found in neuromelanin and iron concentrations in the nigrostriatal system in PD ${ }^{28}$.

Eventually, multiple of these biomarkers may have to be combined to reach a high enough specificity and sensitivity level. Machine learning may play an important role in future work, since models using data of recently diagnosed PD patients can be trained in recognizing PD, which will eventually be able to predict whether someone has PD or not based on multiple imaging features. Such machine learning models however require lots of training data, for which data bases from groups should be combined.

In Chapter 7 we show an important difference in the targeted anatomical location for subthalamic nucleus DBS when targeting on clinical 3T images versus optimized 7T images. Future research should focus on what imaging features drive the neurosurgeons to select a more ventral location when using clinical $3 T$ images, and a less ventral location when using optimized 7T images. It should be studied if the difference in the electrode location is also present when targeting on exactly the same MRI contrasts on both 3T and 7T, since in this study clinically used 3T images (T2) were used while optimized 7T images (T2*, R2*, QSM) were used. Also, future work should focus on whether targeting on 7T images eventually leads to less (cognitive) side-effects compared to targeting on $3 T$ images. The use of ultra-high field imaging in DBS targeting will develop fast over the upcoming years. Of course, it should first be scientifically proven that using 7T MRI images for DBS targeting is leading to a more efficient DBS treatment with less side-effects. After this, 7T MRI images should be used for DBS targeting in patients being suitable for the 7T MRI scanner. Recently, a specific 7T MRI scanner was approved for clinical use (Siemens Magnetom Terra 7T) and this will also speed up research, since research results can in that case be directly translated from science to the clinic, since exactly the same imaging protocols can be used.

\section{Conclusion}

This thesis has provided a deeper insight into the role wearable sensors and MRI can play in tracking and treating PD. It was shown that wearable sensors are definitely feasible to use in the general PD population, and an important step towards wearable sensor validation has been made by combining objective sensor data with subjective questionnaire data. Research in this field has to continue to bridge the gap between the use of home monitoring systems for research purposes towards the clinic. Further, one of the many potential biomarkers for PD (diffusion measures of the olfactory tract) could not track PD. The search for potential biomarkers will continue, since there are many other 
interesting brain structures, like the locus coeruleus, and also many interesting measures, like neuromelanin levels. Finally, this thesis has shown the potential improvement 7T imaging can offer in treating PD using DBS, and has shown that future research should look further into this. To conclude, the deeper insight provided by this thesis gives guidance for future projects using wearable sensors and/or MRI to track and treat PD. 


\section{References}

1. Dorsey, E. R., Sherer, T., Okun, M. S. \& Bloem, B. R. The Emerging Evidence of the Parkinson Pandemic. J. Park. Dis. 8, S3-S8 (2018).

2. Graupe, D. et al. Who May Benefit From On-Demand Control of Deep Brain Stimulation? Noninvasive Evaluation of Parkinson Patients. Neuromodulation J. Int. Neuromodulation Soc. 21, 611-616 (2018).

3. Herron, J. A. et al. Chronic electrocorticography for sensing movement intention and closed-loop deep brain stimulation with wearable sensors in an essential tremor patient. J. Neurosurg. 127, 580-587 (2017).

4. Malekmohammadi, M. et al. Kinematic Adaptive Deep Brain Stimulation for Resting Tremor in Parkinson's Disease. Mov. Disord. Off. J. Mov. Disord. Soc. 31, 426-428 (2016).

5. Jakobs, M., Kloß, M., Unterberg, A. \& Kiening, K. Rechargeable Internal Pulse Generators as Initial Neurostimulators for Deep Brain Stimulation in Patients With Movement Disorders. Neuromodulation J. Int. Neuromodulation Soc. 21, 604-610 (2018).

6. Gilron, R. et al. Long-term wireless streaming of neural recordings for circuit discovery and adaptive stimulation in individuals with Parkinson's disease. Nat. Biotechnol. 1-8 (2021) doi:10.1038/s41587021-00897-5.

7. Dafsari, H. S. et al. Short-term quality of life after subthalamic stimulation depends on non-motor symptoms in Parkinson's disease. Brain Stimulat. 11, 867-874 (2018).

8. Li, D. et al. Remotely Programmed Deep Brain Stimulation of the Bilateral Subthalamic Nucleus for the Treatment of Primary Parkinson Disease: A Randomized Controlled Trial Investigating the Safety and Efficacy of a Novel Deep Brain Stimulation System. Stereotact. Funct. Neurosurg. 95, 174-182 (2017).

9. van der Velden, R. M. J., Mulders, A. E. P., Drukker, M., Kuijf, M. L. \& Leentjens, A. F. G. Network analysis of symptoms in a Parkinson patient using experience sampling data: An $n=1$ study. Mov. Disord. Off. J. Mov. Disord. Soc. 33, 1938-1944 (2018).

10. Vizcarra, J. A. et al. The Parkinson's disease e-diary: Developing a clinical and research tool for the digital age. Mov. Disord. Off. J. Mov. Disord. Soc. 34, 676-681 (2019).

11. Hoff, J. I., Wagemans, E. A. \& van Hilten, B. J. Ambulatory objective assessment of tremor in Parkinson's disease. Clin. Neuropharmacol. 24, 280-283 (2001).

12. Patel, S. et al. Monitoring motor fluctuations in patients with Parkinson's disease using wearable sensors. IEEE Trans. Inf. Technol. Biomed. Publ. IEEE Eng. Med. Biol. Soc. 13, 864-873 (2009).

13. Salarian, A. et al. Quantification of tremor and bradykinesia in Parkinson's disease using a novel ambulatory monitoring system. IEEE Trans. Biomed. Eng. 54, 313-322 (2007).

14. Griffiths, R. I. et al. Automated assessment of bradykinesia and dyskinesia in Parkinson's disease. J. Park. Dis. 2, 47-55 (2012).

15. Habets, J. G. V. et al. A Long-Term, Real-Life Parkinson Monitoring Database Combining Unscripted Objective and Subjective Recordings. Data 6, 22 (2021).

16. Braak, H., Ghebremedhin, E., Rüb, U., Bratzke, H. \& Del Tredici, K. Stages in the development of Parkinson's disease-related pathology. Cell Tissue Res. 318, 121-134 (2004).

17. Postuma, R. B. et al. MDS clinical diagnostic criteria for Parkinson's disease. Mov. Disord. Off. J. Mov. Disord. Soc. 30, 1591-1601 (2015).

18. Atkinson-Clement, C., Pinto, S., Eusebio, A. \& Coulon, O. Diffusion tensor imaging in Parkinson's disease: Review and meta-analysis. Neurolmage Clin. 16, 98-110 (2017).

19. Nigro, P. et al. Changes of olfactory tract in Parkinson's disease: a DTI tractography study. Neuroradiology (2020) doi:10.1007/s00234-020-02551-4.

20. Polders, D. L. et al. Signal to noise ratio and uncertainty in diffusion tensor imaging at 1.5, 3.0, and 7.0 Tesla. J. Magn. Reson. Imaging 33, 1456-1463 (2011).

21. Inglese, M., Fleysher, L., Oesingmann, N. \& Petracca, M. Clinical applications of ultra-high field magnetic resonance imaging in multiple sclerosis. Expert Rev. Neurother. 18, 221-230 (2018).

22. Keuken, M. C., Isaacs, B. R., Trampel, R., van der Zwaag, W. \& Forstmann, B. U. Visualizing the Human Subcortex Using Ultra-high Field Magnetic Resonance Imaging. Brain Topogr. 31, 513-545 (2018).

23. Hershey, T. et al. Mapping Go-No-Go performance within the subthalamic nucleus region. Brain 133, 3625-3634 (2010).

24. McNeely, M. et al. Effects of deep brain stimulation of dorsal versus ventral subthalamic nucleus regions on gait and balance in Parkinson disease. J. Neurol. Neurosurg. Psychiatry 82, 1250-1255 (2011). 
25. Zarzycki, M. Z. \& Domitrz, I. Stimulation-induced side effects after deep brain stimulation - a systematic review. Acta Neuropsychiatr. 32, 57-64 (2020).

26. Schwarz, S. T. et al. Diffusion tensor imaging of nigral degeneration in Parkinson's disease: A region-ofinterest and voxel-based study at $3 \mathrm{~T}$ and systematic review with meta-analysis. Neurolmage Clin. 3, 481-488 (2013).

27. Vaillancourt, D. E. et al. High-resolution diffusion tensor imaging in the substantia nigra of de novo Parkinson disease. Neurology 72, 1378-1384 (2009).

28. Biondetti, E. et al. The spatiotemporal changes in dopamine, neuromelanin and iron characterizing Parkinson's disease. Brain J. Neurol. (2021) doi:10.1093/brain/awab191. 



\section{Addendum}

\section{Impact paragraph}





\section{Contribution to science}

The projects and results described in this thesis contribute to science and society in several ways. First of all, in Chapter 2, we gave an overview of the current available literature about adaptive deep brain stimulation for Parkinson's disease (PD). From the moment this overview was available online, it has been read and has been cited multiple times. We therefore conclude that there indeed was a need for a clear overview about the existing literature and the remaining challenges regarding adaptive deep brain stimulation in PD.

In Chapter 3, the feasibility of the presented home monitoring system is described. For the first time the experience sampling method (questionnaires) and wearable sensors were combined and its feasibility was tested. It is not obvious that such a new system is feasible to use, and that is why this project and the corresponding publication are so important. We have shown to the scientific community that the willingness of PD patients to use such a home monitoring system is huge and that they also are capable of using it. The collected data was made publicly available as described in Chapter 8 and can therefore be used by other research groups all over the world. This contribution may speed up the development of PD symptom algorithms. Chapter $\mathbf{3}$ and Chapter $\mathbf{4}$ both describe a data analysis example and the corresponding results.

In Chapter 5, the protocol of the TRACK-PD study is described. This publication is online and is openly accessible. By publishing this protocol, other groups know what data will be collected for this project, and might get new research ideas or might be interested in collaborations. Chapter $\mathbf{6}$ uses a subset of the data of the project described in Chapter $\mathbf{5}$ and does show a 'negative finding'. This means that we did not find the expected difference between PD patients and healthy controls. Nonetheless, it is important to also publish these results, since this can keep other research groups away from doing identical analyses and encourage them to use different analyzing methods or look into different brain structures. Chapter 6 also gives recommendations for future studies, which might be inspiring for other groups.

The results described in Chapter $\mathbf{7}$ are important for the scientific community since moving towards the use of higher field strength images for deep brain stimulation targeting may result in targeting a different anatomical location within a structure itself. In Chapter $\mathbf{7}$ we describe these results and we do mention that future studies should focus on what this anatomical target difference means. May be the difference means that with a higher field strength the position in the structure can be targeted more precisely, which results in less side-effects for the PD patient when receiving deep brain stimulation. We therefore encourage the scientific community to further look into this, as it might improve future deep brain stimulation treatments. 


\section{Contribution to society}

This thesis has provided a deeper insight into the role wearable sensors and MRI can play in tracking and treating PD. It was shown that wearable sensors are feasible to use in the general PD population, and an important step towards wearable sensor validation has been made. For PD patients, valid home monitoring would mean that PD symptoms can be tracked more accurately over time, which may result in an improved adjustment of PD medication and/or deep brain stimulation settings. Also, home monitoring can give PD patients the possibility for remote care, since their PD symptoms can be tracked from a distance. This is particularly beneficial for PD patients with a reduced mobility and for patients with poor access to nearby medical facilities in other regions of the world. Moreover, the feasibility of home monitoring as shown for PD patients also encourages this approach for other diseases with reduced mobility. In addition, a reduction of hospital visits enabled by home monitoring will have a beneficial economical effect for society. The results of this thesis show progress regarding the diagnostic work-up for PD. As mentioned in the introduction, the diagnostic work-up of PD can be an endless process, which often entails a long period of insecurity for the patient and his or her loved ones. If a MRI biomarker is found for PD, this insecurity will be much less. In addition, the invasive and time consuming DaT-SCAN may become unnecessary and less visits to the outpatient clinic and second opinion visits elsewhere might be necessary. This not only is more comfortable for the PD patient, it again also may eventually have a beneficial economical effect. Finally, this thesis has provided new insights into the use of 7T imaging for deep brain stimulation targeting. Further research should continue in this field, since more precise targeting might lead to less side-effects, which in the end leads to an improved quality of life for the PD patient.

This thesis is relevant for everyone, since PD, as already mentioned before, has a rapidly increasing incidence and prevalence. Not only by increasing the awareness of PD and PD symptomatology, but also by the awareness of the current research developments. This will also be beneficial for the PD patient population, since they of all people know that PD is not 'just a shaking hand disease', and a higher awareness of PD by the general population will increase understanding towards the PD patient. The results of this these are, or will be, published online in international scientific journals. These publications are often hard to interpret and are not available in Dutch. The Parkinson Vereniging therefore translates and simplifies interesting publications to make it more accessible for the broader PD audience. A simplified and Dutch summary of Chapter $\mathbf{3}$ for example was published on their website (https://www.parkinson-vereniging.nl/archief/bericht/2019/11/01/Het-monitoren-van-

Parkinson-symptomen-in-het-dagelijks-leven-Een-haalbaarheidsstudie). Participants of the TRACK-PD study also receive a Dutch summary of the results in the TRACK-PD 
newsletter. We inform participants about study outcomes, since we think it is an important way of showing our appreciation to them and since the results are relevant to them. 



\section{Addendum}

Curriculum Vitae 

Margot Heijmans was born on the $27^{\text {th }}$ of October 1994 in Venlo, The Netherlands. After completing pre-university education at the Bouwens van der Boijecollege in Panningen in 2012, she started studying Human Movement Sciences at the Vrije Universiteit in Amsterdam. During this bachelor she specialized in a minor Biomolecular- and Neuroscience (track Neuroscience) and performed her Bachelor Research project 'left/right recognition in active runners experiencing unilateral achilles tendon inflammation'. She completed her bachelor's degree in Human Movement Sciences in 2015. She then enrolled in the Master's programme in Cognitive Neuroscience at the Radboud University in Nijmegen. During this master she chose the Perception, Action, and Control track and graduated in 2017. Her master thesis 'Structural integrity of midbrain nuclei in tremor-dominant and non-tremor Parkinson's disease was supervised by Dr. Rick Helmich and Dr. Annelies van Nuland. In 2017 she started her PhD project under the supervision of Dr. Pieter Kubben, Dr. Mark Kuijf and Prof. Dr. Yasin Temel. This thesis is a result of that PhD project. After almost four years of full-time research at the Maastricht University, Margot started working parttime ( $0.6 \mathrm{fte})$ as research coordinator at the surgery department of the Máxima MC in Veldhoven. Besides, she finished her PhD project and will keep on working parttime $(0.4 \mathrm{fte})$ as a post-doc on the TRACK-PD study at the Maastricht University. 



\section{Addendum}

PhD portfolio 



\section{Published articles in this thesis}

Isaacs, B. R.* Heijmans, M*., Kuijf, M.L., Kubben, P.L., Ackermans, L., Temel, Y., Keuken, M.C., Forstmann, B.U. (2021). Variability in subthalamic nucleus targeting for deep brain stimulation with 3 and 7 Tesla magnetic resonance imaging. Neuroimage: Clinical.

Wolters A.F., Heijmans M., Michielse S., Leentjens A.F.G., Postma A.A., Jansen J.F.A., Ivanov D., Duits A.A., Temel Y., Kuijf M.L. (2020). The TRACK-PD study: protocol of a longitudinal ultra-high field imaging study in Parkinson's disease. BMC Neurology.

Heijmans, M., Habets, J. G. V., Herff, C., Aarts, J., Stevens, A., Kuijf, M. L., Kubben, P. L. (2019). Monitoring Parkinson's disease symptoms during daily life: a feasibility study. Nature Partner Journal: Parkinson's disease.

Heijmans, M*., Habets, J. G. V*., Kuijf, M. L., Kubben, P. L., Herff, C. (2019). Evaluation of Parkinson's disease at home: Predicting tremor from wearable Sensors. IEEE Engineering in Medicine and Biology Society.

Habets, J. G. V*., Heijmans, M*., Kuijf, M. L., Janssen, M. L., Temel, Y., \& Kubben, P. L. (2018). An update on adaptive deep brain stimulation in Parkinson's disease. Movement Disorders.

\section{Submitted articles in this thesis}

Heijmans, M., Wolters, A.F., Temel, Y., Kuijf, M. L., Michielse, S. Comparison of olfactory tract diffusion measures between early Parkinson's disease patients and healthy controls using ultra-high field MRI.

\section{Other articles}

Habets, J. G., Heijmans, M., Leentjens, A. F., Simons, C. J., Temel, Y., Kuijf, M. L., Kubben, P.L., Herff, C. (2021). A long-Term, real-life Parkinson monitoring database combining unscripted objective and subjective recordings. Data.

Habets, J., Heijmans, M., Herff, C., Simons, C., Leentjens, A.F.G., Temel, Y., Kuijf, M., Kubben, P. (2020). Mobile health daily life monitoring for Parkinson disease: Development and validation of ecological momentary assessments. JMIR Mhealth Uhealth.

Beudel, M., Heijmans, M., Habets, J. G., \& Kubben, P. L. (2020). Future perspectives: Adaptive deep brain stimulation. In Fundamentals and Clinics of Deep Brain Stimulation (pp. 49-65). Springer.

*shared first authors 


\section{Courses}

- $\quad 3^{\text {rd }}$ Clinical Neuroscience Course

- Samsun, Turkey, 2017

- Experience Sampling Course

o Maastricht University, The Netherlands, 2017

- Experience Sampling Data Analysis Course

○ Maastricht University, The Netherlands, 2018

- Multilevel Analysis of Longitudinal Data

- Maastricht University, The Netherlands, 2018

- Introduction to problem-based learning

○ Maastricht University, The Netherlands, 2018

- Self-management for PhD projects

○ Maastricht University, The Netherlands, 2018

- Tutor training

○ Maastricht University, The Netherlands, 2019

- Deep Brain Stimulation Fundamental Course

○ Abbott, Dusseldorf, Germany, 2018

- Basiscursus Regelgeving en Organisatie voor Klinisch onderzoekers

○ Maastricht, The Netherlands, 2018

- Magnet Safety Training

○ Scannexus, Maastricht, The Netherlands, 2019

- $\quad$ Certified User Authorisation for the 7T MRI system

○ Scannexus, Maastricht, The Netherlands, 2019

- Basis Kwalificatie Onderwijs

○ Maastricht University, Maastricht, The Netherlands, 2020 


\section{Conferences}

- $\quad$ EURON PhD Days

○ Leuven, Belgium, 2018

- European Society for Stereotactic and Functional Neurosurgery

○ Edinburgh, United Kingdom, 2018

- Annual International Conference of the IEEE engineering in Medicine and Biology Society

○ Berlin, Germany, 2019

- International Course on Neuromodulation

○ Maastricht, The Netherlands, 2019

- International Congress of Parkinson's Disease and Movement Disorders

○ Nice, France, 2019

- Neuroimaging and Neurophysiology of Movement Disorders

- MDS-ES, Brno, Czech Republic, 2019

\section{Teaching}

- Tutor BBS1004, Brain, Behavior and Movement

- Tutor GEN1004, Denken en Doen

- Tutor PSY4320, Fundamental Neuroscience

- Tutor MBS1601, Fundamental Neuromodulation

- Supervisor Human brain anatomy practical classes

- Supervisor Sheep brain anatomy practical classes

- Supervision Bachelor Research Projects 



\section{Addendum}

Nederlandse samenvatting 

De hoofdstukken in dit proefschrift beschrijven onderzoek naar het opsporen en behandelen van de ziekte van Parkinson. Bij velen zal er een belletje gaan rinkelen bij het horen van 'de ziekte van Parkinson'. Dit is niet vreemd, gezien de ziekte van Parkinson ook wel de snelst groeiende hersenziekte in de wereld wordt genoemd, welke uit kan monden in een ware 'Parkinson-pandemie'. Omdat er nog zoveel onbekend is over de ziekte van Parkinson en omdat iedere patiënt met de ziekte van Parkinson anders is, is het uitermate belangrijk om hier onderzoek naar te doen.

Momenteel kunnen patiënten met de ziekte van Parkinson behandeld worden middels medicatie en, mocht medicatie niet goed of niet meer werken, dan ook via diepe hersenstimulatie. Dit is een behandeling waarbij electroden in de hersenen van de patiënt worden geplaatst en een soort van pacemaker in de borst. Door het stimuleren van bepaalde hersengebieden worden Parkinson symptomen onderdrukt.

Patiënten met de ziekte van Parkinson worden slechts om de paar maanden door hun neuroloog of Parkinson verpleegkundige gezien. Dit heeft een aantal nadelen. Ten eerste is dit bezoek slechts een momentopname en zal de patiënt moeten beschrijven hoe het de afgelopen maanden ging. Dit zal tot 'recall bias' leiden; het onnauwkeurig beschrijven van informatie gezien het over de afgelopen vaak lange periode gaat. Verder kan het zijn dat de patiënt zich beter voordoet bij de neuroloog of verpleegkundige dan hij/zij zich eigenlijk voelt en kan de neuroloog of verpleegkundige slechts een subjectieve beschrijving geven van hoe het gaat met de patiënt. Als resultaat zal de medicatie en/of de instellingen voor de diepe hersenstimulatie mogelijk suboptimaal aangepast worden.

Door deze tekortkomingen in de behandeling van de ziekte van Parkinson komt er steeds meer interesse in thuismonitoring. Dit houdt in dat patiënten tijdens hun alledaagse bezigheden bijvoorbeeld bewegingssensoren dragen en dat deze scores geven over hoe het met de Parkinson symptomen gaat. Eventueel kan dit nog gecombineerd worden met vragenlijsten, zodat ook de niet bewegingssymptomen in kaart gebracht kunnen worden. Als de patiënt vervolgens op bezoek komt bij de neuroloog, kan deze inzicht krijgen in het verloop van de klachten en kan de neuroloog ook de verschillen tussen dagen en de verschillen gedurende één dag zien. Zo kunnen de medicatie en/of instellingen voor de diepe hersenstimulatie mogelijk effectiever worden afgestemd.

De hoofdstukken in het eerste deel van dit proefschrift beschrijven hoe draagbare bewegingssensoren bij kunnen dragen aan met name het behandelen van de ziekte van Parkinson. In hoofdstuk $\mathbf{2}$ staat een update beschreven over diepe hersenstimulatie bij de ziekte van Parkinson en meer specifiek over adaptieve diepe hersenstimulatie. Dit houdt in dat de instellingen van de diepe hersenstimulatie niet om de paar maanden door de specialist in het ziekenhuis worden aangepast, maar dat de instellingen vanzelf worden aangepast aan de hand van metingen die representeren hoe het met de symptomen van 
de patiënt gaat. Onderzoek heeft aangetoond dat draagbare bewegingssensoren een rol in adaptieve diepe hersenstimulatie kunnen spelen. Sensoren meten dan bijvoorbeeld hoeveel een patiënt met de ziekte van Parkinson trilt en kunnen op het moment dat het trillen erger is de hersenen meer stimuleren, zodat het trillen ook meer onderdrukt wordt. De uitdaging van adaptieve diepe hersenstimulatie met bewegingssensoren blijft echter dat de stijfheid en traagheid van bewegen moeilijker te meten zijn met deze sensoren. Onderzoek zal moeten uitwijzen of dit mogelijk is, of dat het nodig is dat de signalen van de bewegingssensoren met andere te meten signalen gecombineerd dienen te worden.

In hoofdstuk 3 van dit proefschrift is ons onderzoek beschreven waarin 20 patiënten met de ziekte van Parkinson ons thuismonitoring systeem getest hebben. Dit systeem bestond uit drie draagbare sensoren (een op iedere pols en een op de borst) en een applicatie met vragenlijsten op de telefoon van de deelnemer. Deze applicatie stuurde zeven keer per dag een vragenlijst naar de deelnemers, welke deze vragenlijst binnen een kwartier moesten openen om in te vullen. Bij de vragen ging het niet alleen om het scoren van bewegingssymptomen van de ziekte van Parkinson, maar ook juist om de nietbewegingssymptomen van de ziekte van Parkinson, gezien deze niet met sensoren te meten zijn. We hebben met deze studie aangetoond dat dit systeem intensief gebruikt werd en door de deelnemers als gebruiksvriendelijk werd ervaren. Verder is in dit hoofdstuk een voorstel beschreven hoe we deze nieuwe combinatie van data van sensoren en data uit vragenlijsten kunnen combineren. Als laatste hebben we aangetoond dat het vrij goed mogelijk was om aan de hand van data van de bewegingssensoren van één deelnemer, te voorspellen of deze deelnemer 'ON' of 'OFF' was. De deelnemer gaf in de vragenlijsten aan $\mathrm{ON}$ te zijn wanneer hij/zij het gevoel had dat de medicatie goed werkte en OFF wanneer hij/zij het gevoel had dat dit niet zo was. In hoofdstuk 4 hebben we laten zien dat het mogelijk was om aan de hand van data van de bewegingssensoren van één deelnemer, de door de deelnemer gegeven tremor score (ernst van het trillen) te voorspellen. Dit deden we met de data van het kwartier voorafgaande aan het moment dat de patiënt het trillen scoorde middels de vragenlijst. De bovengenoemde analyse toont aan dat het mogelijk is om met de objectieve gegevens van de bewegingssensoren de subjectieve tremor score te voorspellen, welke gegeven werd door de deelnemer zelf. Hieruit kunnen we concluderen dat het mogelijk is om aan de hand van de objectieve gegevens van de sensoren te bepalen hoe het met de symptomen van de patiënt gaat, wat zeer waardevol is voor thuismonitoring.

De hoofdstukken in het tweede deel van dit proefschrift beschrijven hoe MRI scans (oftewel afbeeldingen van de hersenen) bij kunnen dragen aan het opsporen en behandelen van de ziekte van Parkinson. Het opsporen, ofwel het diagnosticeren, van de ziekte van Parkinson wordt momenteel gedaan door de klachten van de patiënt te beschouwen en door de eventuele verandering van klachten over de tijd te bekijken. Vaak 
wordt een MRI scan in het diagnostische proces ook gemaakt om eventuele andere oorzaken voor de klachten, zoals een hersentumor, uit te sluiten. Verder kan bij twijfel over de diagnose ook nog een invasieve DaT-scan worden gemaakt, waarbij gebruik wordt gemaakt van een radioactieve vloeistof.

In hoofdstuk $\mathbf{5}$ is het protocol beschreven van de TRACK-PD studie. Dit is een longitudinaal onderzoek, waarbij er in totaal drie testdagen zijn voor iedere deelnemer; op het begin, na 2 jaar en na 4 jaar. In deze studie doen controle deelnemers mee en patiënten welke maximaal 3 jaar geleden gediagnosticeerd zijn met de ziekte van Parkinson. Wij zijn in de zomer van 2019 gestart met de uitvoering van dit onderzoek. Twee jaar later hebben er ongeveer 150 deelnemers meegedaan en zijn de eerste deelnemers al voor de tweede keer terug gekomen. De testdagen van dit onderzoek bestaan uit een (sterke) 7 Tesla MRI scan, vragenlijsten over klachten die kunnen voorkomen bij de ziekte van Parkinson, eenmalige bloedafname, bewegingstesten en neuropsychologische testen. Het doel van dit onderzoek is om uit te zoeken of men door het maken van een MRI-scan van de hersenen, vast kan stellen of iemand de ziekte van Parkinson heeft of niet. Daarnaast willen we onderzoeken of groepen patiënten met verschillende Parkinson symptomen, of welke verschillende Parkinson symptomen ontwikkelen over de tijd, ook verschillende soorten veranderingen op de MRI-scan laten zien. Dit zou ons kunnen helpen om de ziekte beter te voorspellen en bovendien de behandeling beter aan te kunnen passen aan de individuele patiënt.

In hoofdstuk 6 van dit proefschrift is ons onderzoek beschreven waarin we kijken of we met behulp van een bepaalde soort MRI scan, de diffusie gewogen ofwel DWI scan, verschillen zien in de reukzenuw tussen de controle groep en de Parkinson groep van de TRACK-PD studie. De reukzenuw is een structuur die als een van de eerste structuren is aangedaan bij de ziekte van Parkinson. Het is dan ook niet gek dat veel patiënten met de ziekte van Parkinson op het moment van diagnose vaak al jaren een minder goede reuk ervaarden. De reukzenuw is daarom één van de structuren welke geschikt lijkt te zijn om Parkinson aan te tonen, aangezien deze vaak al in een vroeg stadium aangetast is. Eerdere studies welke ook met behulp van de DWI scan naar de reukzenuw keken vonden verschillen in DWI waardes tussen de controle groep en de Parkinson groep. Dit waren groepen waarbij was aangetoond dat de Parkinson groep slechter rook en geuren kon onderscheiden dan de controle groep. Wij wilden met deze studie juist kijken of het mogelijk is om ongeacht de ruikcapaciteit van de deelnemers een verschil aan te tonen in de DWI waardes van de reukzenuw. Dit omdat, willen deze DWI waardes van de reukzenuw geschikt zijn om Parkinson aan te tonen, dit niet afhankelijk moet zijn van de reukcapaciteit. Tegenover eerdere studies was dit de eerste studie die naar de reukzenuw keek met 7 Tesla MRI scans in vergelijking met 3 Tesla MRI scans. 7 Tesla MRI scans kunnen preciezere beelden geven, maar het is onduidelijk of dit ook geldt voor de DWI scans, gezien deze gevoeliger zijn voor verstoringen. We hebben aangetoond met deze studie dat we geen 
verschillen vonden in de DWI waardes van de reukzenuw tussen de controle groep en de Parkinson groep. We hebben daardoor geconcludeerd dat 7T DWI waardes van de reukzenuw niet geschikt zijn om Parkinson aan te tonen. Dit wil niet uitsluiten dat de reukzenuw geen geschikte structuur is om Parkinson aan te tonen. Verder onderzoek zal moeten uitwijzen of andere bepalingen van de reukzenuw of DWI waardes van andere structuren mogelijk wel geschikt zijn om Parkinson aan te tonen.

Hoofdstuk 7 van dit proefschrift richt zich op het optimaliseren van diepe hersenstimulatie bij de ziekte van Parkinson. Momenteel worden de electroden bij de ziekte van Parkinson met name in de subthalamic nucleus (STN) geplaatst. Dit is een amandelvormige hersenstructuur met een grootte van $20-30 \mathrm{~mm}^{3}$. De exacte plaatsing van de electroden binnen deze structuur is zeer belangrijk. We weten namelijk dat een suboptimale plaatsing kan leiden tot ongewenste (cognitieve) bijwerkingen. Momenteel wordt de exacte plaatsing bepaald aan de hand van een 3 Tesla MRI scan. Ondanks dat we weten dat een 7 Tesla (sterkere) MRI scan kan leiden tot nauwkeurigere plaatjes met een resolutie van minder dan een millimeter, weten we niet of het gebruik van 7 Tesla MRI scans leidt tot een verschil, en mogelijk een verbetering, in het plaatsen van de electroden in de STN voor diepe hersenstimulatie bij de ziekte van Parkinson. Om dit te onderzoeken hebben wij drie neurochirurgen herhaaldelijk de volgens hen optimale plek van stimulatie binnen de STN laten bepalen op 3T en 7T MRI scans. Ze hebben dit ieder vijfmaal gedaan op vier verschillende scans (één 3T scan en drie verschillende soorten 7T MRI scans) van vijf Parkinson patiënten voor zowel de linker als rechter STN. Uit dit onderzoek kwam naar voren dat de neurochirurgen consistent zijn in het kiezen van de optimale plek van stimulatie en dat dit niet beïnvloed werd door de sterkte van de MRI scan en het type MRI scan. Wanneer we de coördinaten van de aangewezen plekken in een standaard coördinatenstelsel plaatsten (in plaats van in de persoon specifieke omgeving), zagen we echter dat er wel verschillen zijn in de coördinaten geplaatst op 3T MRI scans en 7T MRI scans. We zagen dat de coördinaten van de uitgekozen plek bij 3T MRI scans meer ventraal, dus naar voren, geplaatst werden in vergelijking met de coördinaten van de 7T MRI scans. Het is nog onbekend wat precies voor deze verschillen zorgt en welk effect deze andere plaatsing heeft op de effectiviteit van DBS in Parkinson. Dit onderzoek is dan ook een interessante aanzet voor vervolg onderzoek.

Als laatste zijn in hoofdstuk $\mathbf{8}$, het discussie hoofdstuk, de belangrijkste bevindingen uit de eerdere hoofdstukken samengevat en wordt er hier dieper ingegaan op methodologische aspecten van de beschreven studies. Verder worden hier aanbevelingen voor toekomstige studies beschreven. 


\section{Addendum}

Dankwoord 

Ten eerste wil ik natuurlijk mijn promotor Yasin Temel en co-promotoren Mark Kuijf en Pieter Kubben bedanken voor de kans die jullie me hebben gegeven om deze mooie projecten uit te mogen voeren. Jullie hebben me laten zien dat het niet niks is om naast een baan als succesvolle clinicus ook nog onderzoek uit te voeren en hiernaast ook nog eens vader te zijn. Ik wil jullie enorm bedanken voor onze fijne samenwerking.

Vervolgens wil ik graag mijn paranimfen Perla en Amée bedanken.

Perla. Augustus 2017 in de koffieruimte. Huhhhhh die ken ik toch? Ja hoor. Het was Perla, die ik al kende van de bachelor Bewegingswetenschappen. Hoewel we elkaar nog niet echt goed kende toen we allebei in Amsterdam studeerden, hebben we ons hier in Maastricht toch wel heel snel goed leren kennen! Van onze etentjes tot vlaaimomentjes op kantoor, ik wil je bedanken voor alle leuk tijd samen! Ik wens je heel veel succes met het afronden van je PhD en ik hoop dat je in de komende periode een baan vind waarin je je creativiteit op een enthousiaste manier kwijt kunt.

Amée. Terwijl ik er op dat moment nog weinig vanaf wist, ben jij begonnen met het opzetten van de TRACK-PD studie. Ik waardeer enorm hoe jij dit op hebt gezet en ik ben erg blij dat ik later aan mocht sluiten bij het praktisch realiseren van deze studie. Vanaf 2019 hebben we elkaar zowel op het werk als privé goed leren kennen. Ik wil je bedanken voor de gezelligheid op kantoor, je luisterende oor en de enorm fijne samenwerking. Verder wil ik mijn bewondering uitspreken voor jou harde werken en het combineren van je AIOS neurologie met je PhD. Ik ben blij dat ik degene was die af en toe mocht zeggen 'NEE je gaat niet werken je hebt vrij'. Ik wil je heel veel succes wensen met de laatste loodjes van je PhD en natuurlijk van je specialisatie, maar dat gaat jou zeker lukken!

Vervolgens wil ik graag de neurochirurgie research groep bedanken. Ik kwam binnen bij deze onderzoeksgroep zonder dat ook maar iemand wist dat ik zou gaan beginnen, maar toch werd ik al snel onderdeel van deze onderzoeksgroep. Bedankt voor de gezellige lab uitjes, meetings, schapenbrein practica en de opgedane kennis. Verder ga ik geen hele lijst opnoemen met alle collega's van de afdeling die ik wil bedanken. Ik wil namelijk gewoonweg iedereen van 'de gang' bedanken voor de klets momenten, de gezellige lunchpauzes en de eeuwige tosti lucht! Een aantal collega's wil ik wel nog even speciaal bedanken:

Mark Janssen, Ali, Sarah en Govert. Ik wil jullie, als seniors van de neurochirurgie groep, bedanken voor de fijne samenwerkingen. Ook bedankt voor de werk gerelateerde adviezen, gesprekken over 'wat na mijn PhD' en de ervaring in het geven van onderwijs die ik in jullie blokken heb mogen opdoen. 
Christian. You joined our research group in September 2018. You know I was really glad with that, since I saw you as the savior of the sensor work. I really appreciate working with you and the results of our collaborations. I think you are THE role model of how a scientist should be; super knowledgeable, professional and last but not least very approachable. I really appreciate our conversations and your wise advices. I wish you all the luck in the world, and hope to see you soon as Prof. Herff.

Jeroen. We begonnen tegelijkertijd onze PhD bij Pieter en Mark. Ik vond het fijn dat we op het begin samen zoekende waren welke richting op te gaan. Wie had dat gedacht, eerst naar Turkije presenteren over aDBS (jaja, met tolk!) en een tijdje later samen onze succesvolle review gepubliceerd hebben! Bedankt voor de fijne samenwerking en gezelligheid op kantoor. Ik wens je heel veel succes en liefde in Berlijn!

Sylvana. Jij bent een maandje voor mij begonnen binnen de neurosurgery groep. Ik heb je leren kennen als een harde werker, die zegt waar het op staat. Stiekem was ik hier wel eens jaloers op. Mede dankzij jou heb ik nog meer respect gekregen voor alle collega's die met proefdieren werken, want ojojoj wat een gedoe af en toe! Bedankt voor de fijne carpool momenten, gezelligheid in ons kantoor en natuurlijk je luisterende oor. Ik bewonder je lef om de keuze te maken om uiteindelijk te vertrekken. Ik wens je al het goeds met je huidige baan en ik wil jou, Wesley en Guus heel veel geluk wensen in jullie nieuwe crib in Raalte, wat op een dag toch echt ingeruild zal gaan worden voor die droom boerderij!

Jana. Jij begon in 2018 bij ons als PhD student binnen de tinnitus tak. Ik wil je bedanken voor de gezelligheid in ons kantoor en soms ook de chaos haha. Ook wil ik je bedanken voor het opkrikken van mijn Belgische woordenschat, waar ik samen met Amée en Sylvana af en toe toch wel om moest lachen. Ik bewonder hoe je de combinatie met het werken op de KNO afdeling en in het lab tot stand hebt gebracht en wil je al het goeds wensen voor de toekomst!

Glenn. Ik wil je bedanken dat je mij al één keer hebt laten ervaren hoe het is om voorin de aula te staan, weliswaar als jou paranimf! Verder natuurlijk voor de gezellige borrels (met bijbehorende lijstjes..), de goede gesprekken, je film quotes, en natuurlijk de etentjes bij Perla en jou thuis!

Maarten, neurotechnoloog. Hah de oude bekende. In 2012 begonnen we allebei als kinders in het Vondelpark aan de introductieweek van Bewegingswetenschappen, jij als kangoeroe, ik als papegaai. Daarna heb je mij met name leren kennen als ***FIC en ik jou als een enthousiaste intropappa voor de nieuwe lichting giraffes en olifantjes. Bewondering hiervoor! In april 2019 hadden we ineens weer contact over een vacature hier in Maastricht en een tijdje later waren we collega's. Ik wil je bedanken voor de gezelligheid en voor de 
fijne gesprekken over zowel werk als alledaagse zaken. In het speciaal natuurlijk ook bedankt voor de digitale koffie pauzes tijdens al het thuiswerken!

Stijn. Ik wil je bedanken voor onze fijne samenwerking voor de TRACK-PD studie. Je hebt me enorm veel geleerd over MRI en de analyses hiervan. Ik ben blij met, en trots op, het resultaat van onze samenwerking. Bedankt ook voor de gezellige 200+ uren samen scannen en bedankt dat ik altijd hierbij op je kon rekenen. Heel veel succes met je carrière, maar dat komt zeker goed!

Chris. Thanks for making me and the other colleagues of our hallway realizing that it was already time for lunch! Furthermore, thanks for our nice conversations and tea moments and of course our shared initiation as paranymphs of Glenn.

Roman. Bedankt voor het up-to-date houden van mijn dumpert kennis en sportuitslagen en voor de gezelligheid op 'het voorste' kantoor. Ik wens je heel veel succes met Italiaans leren, het vader zijn, het afronden van je PhD en natuurlijk je (hopelijk) toekomstige opleiding tot chirurg Assmann!

Bethany. I got to know you as 'the one who slams on the keyboard accompanied with some swear words'. After that, I really got to know you, which resulted in nice, good, and sometimes heavy skype conversations. I'm proud of the results of our joined project and wish you all the best in your future career!

Lonne. Bedankt voor de gezellige carpool momenten met veel geklets, werk geklets en af en toe lekkere dutjes.

Rose. Bedankt voor al de gezelligheid en je aanstekelijke lach! Ook natuurlijk nog voor de Porto tips! Heel veel succes met het vinden van je volgende uitdaging.

Natuurlijk zijn er ook een heel aantal mensen buiten het werk die ik wil bedanken voor alles! Bedankt Joey, Honingdropjes, Egchel en un bietje Near, volleybal teamgenootjes, Niels, Marissa, collega's bij het Máxima MC en de rest!

Pap en mam. Bedankt voor jullie steun. Ik weet dat ik altijd op jullie kan rekenen, wat er ook gebeurt. Bedankt ook dat ik gedurende mijn PhD weer even terug in het thuisfront mocht komen wonen. Ik besef achteraf pas dat ook dit zeker niet vanzelfsprekend is en dat voor jullie een tweede 'lege nest syndroom' ook niet makkelijk zal zijn geweest. Verder bedankt voor jullie interesse in mijn werk, ook al was het soms wat moeilijk te begrijpen allemaal. 
Christel. Jij ook bedankt voor je steun en voor het advies om naar mezelf te luisteren en mijn eigen gevoel te volgen. Ik bewonder hoe jij zo goed bent in het 'doen waar je zin in hebt en hoe dat gaat zien we dan wel'. Gezien je niet om de hoek woont, bedankt voor de videobelletjes en de app gesprekken.

Laureen. Jij uiteraard ook bedankt voor al je steun de afgelopen jaren. Bedankt dat ik een periode deel uit mocht maken van jullie interieur (volgens Rick dan). Bedankt ook voor de leuke bootcamp momenten samen en tijdens de lockdown ook de hardloop sessies (of zullen we toch wandelen? ha-ha).

Peter. Je bent de beste en liefste buur-buur die ik me ooit had kunnen wensen. Ik hou van jou!

Als laatste wil ik mijn enorme dankbaarheid uitspreken voor alle deelnemers aan ons onderzoek. Zonder deze deelnemers was dit boekje er natuurlijk niet geweest. Ik wil de deelnemers bedanken voor hun inzet, geduld en medewerking tijdens de testdagen, maar ook voor hun gezelligheid en interesse in het onderzoek. Dank jullie wel! 